University of Tennessee Health Science Center

UTHSC Digital Commons

\title{
Pitx3Null Mutant (Striatal Dopamine-Deficient) Mice Have Exaggerated Spiny Projection Neuron Responses to I-DOPA and D1 Agonism and Lack Baseline Striatonigral Spiking
}

\author{
Ben Sagot \\ University of Tennessee Health Science Center
}

Follow this and additional works at: https://dc.uthsc.edu/dissertations

Part of the Medical Biochemistry Commons, Medical Neurobiology Commons, Nervous System Diseases Commons, and the Neurology Commons

\section{Recommended Citation}

Sagot, Ben (https://orcid.org/0000-0002-9141-4369), "Pitx3Null Mutant (Striatal Dopamine-Deficient) Mice Have Exaggerated Spiny Projection Neuron Responses to I-DOPA and D1 Agonism and Lack Baseline Striatonigral Spiking" (2017). Theses and Dissertations (ETD). Paper 447. http://dx.doi.org/10.21007/ etd.cghs.2017.0442.

This Thesis is brought to you for free and open access by the College of Graduate Health Sciences at UTHSC Digital Commons. It has been accepted for inclusion in Theses and Dissertations (ETD) by an authorized administrator of UTHSC Digital Commons. For more information, please contact jwelch30@uthsc.edu. 


\title{
Pitx3Null Mutant (Striatal Dopamine-Deficient) Mice Have Exaggerated Spiny Projection Neuron Responses to I-DOPA and D1 Agonism and Lack Baseline Striatonigral Spiking
}

\begin{abstract}
L-3,4 dihidroxyphenylalanine (I-DOPA) strongly stimulates motor activity in parkinsonian patients and animal models of Parkinson's disease. Severe striatal dopamine (DA) loss characterizes Parkinson's disease and its animal models. Given the canonical rate model of Parkinson's Disease pathophysiology based on differences in DA pharmacology manifesting as electrophysiological differences in striatal projection neuron (SPN) spike rates, SPNs should increase spiking during the motor response to I-DOPA. In fact, stimulating specific subsets of these neurons to spike in freely-moving wild type and parkinsonian animals causes or inhibits motor activity as predicted. However, pharmacological effects of DA deficiency, let alone those of DA replacement, on SPN spiking activity in freely-moving animals are poorly studied and ultimately unknown. Showing the activity of SPNs of both in-/direct pathways may help elucidate mechanisms by which I-DOPA increases motor activity to normal and sometimes abnormal levels; such mechanistic information would advance understanding about how DA is such a potent motor stimulant.

To this end, I devised a Top-hat u-array (with microdrive) for recording in the striatum while stimulating the reticulated substantia nigra. Using my micro-array, I tested I-DOPA's acute effect on SPN spiking activity within contexts that varied in DA deficiency according to the Pitx3Null mouse's Parkinson's-like gradient of striatal DA denervation. Evidently, chronic DA denervation renders SPNs hyper-responsive to IDOPA and a D1 agonist, SKF 81297, as indicated by exaggerated SPN spike rate responses biased by low baselines in Pitx3Null mice compared to wild-type mice. However, this may be a motor network effect on spiking as it was found in both dorsal (DA-denervated) and non-dorsal (having residual DA) Pitx3Null striatal regions. Furthermore, antidromically identifying dorsal SPNs allowed us to putatively distinguish a particularly relevant subset (striatonigral, D1-SPNs or d[irect]SPNs) known to elicit movement; serendipitously we also identified putative fibers of passage that strongly resembled striatal interneurons. D1-SPNs in Pitx3Null animals had baselines about an order of magnitude significantly below those in wild-type, and all increased firing more so in Pitx3Null than wild-type mice after drug injections, which lends some credence to the hypothesis that direct pathway SPNs are hyper-responsive during I-DOPAinduced normal and abnormal motor behavior secondary to DA depletion. Furthermore, they uncover a need to incorporate more neural factors in explaining electrophysiological effects attributable to DA denervation and restoration pharmacology. The latter data (putative fibers) tempt the interpretation that cortical axons of passage are being mistaken for striatal fast-spiking interneurons in the literature more often than not.
\end{abstract}

\section{Document Type}

Thesis

\section{Degree Name}

Master of Science (MS)

Program

Biomedical Sciences

Research Advisor

Fu-Ming Zhou, M.D., Ph.D. 


\section{Keywords}

striatal, antidromic, electrophysiology, Pitx3Null, fast-spiking, dopamine, Parkinson's, spiny, freely-moving

\section{Subject Categories}

Medical Biochemistry | Medical Neurobiology | Medical Sciences | Medicine and Health Sciences |

Nervous System Diseases | Neurology 
Pitx3Null Mutant (Striatal Dopamine-Deficient) Mice Have Exaggerated Spiny Projection Neuron Responses to l-DOPA and D1 Agonism and Lack Baseline Striatonigral Spiking

\author{
A Thesis \\ Presented for \\ The Graduate Studies Council \\ The University of Tennessee \\ Health Science Center \\ In Partial Fulfillment \\ Of the Requirements for the Degree \\ Master of Science (Under Protest) \\ From The University of Tennessee
}

By

Ben Sagot

December 2017 
Copyright (C) 2017 by Ben Sagot. All rights reserved. 


\section{DEDICATION}

Strictly speaking this work is dedicated to the community that finds it most valuable. I do not expect my family, friends and romances to care much about this work, but they certainly empowered me to continue when it seemed that doing so might not be worth it.

My advisor, Fu-Ming Zhou, dissertation committee members, Kazuko Sakata, Jeff Steketee, Kristen O'Connell, and Wen-Lin Sun, and my past advisor, Detlef Heck, might benefit from perusing this work. They might not. 


\section{ACKNOWLEDGEMENTS}

A double-handed thankyou to all my mentors: those who taught me at times from their knowledge (some people more often than others, Bob Waters) and at other times from their ignorance (some others more often than the rest...).

I got years of emotional, moral, technical help to continue this work; I need to thank those who eased the tensions of everyday life, most especially Momma Hen and Rooster Mike, old romantic roommie Lauryn Spielvogel (sometimes just in memory), Trusted Advisor Bill Veeser, others I met along the way that spiced up the entire experience: Eliza, Kyle, WeiWei, Qiu, LiLi, Sean, Andy, Afshin, Udita, Felisa, David, Ikbale, Hollyanne, Brittany, Natasha, Malik lab members (Nayaab, Ajeeth, Kamalika, Purnima, Chi-young, Ji-soo)... surely others I've forgotten here. Jackie inspired peace of mind I could never have had by myself without going the full nine on a whole "nother degree, which made this entire transition out of grad school free of the regret of wondering what could have been if I had just put in more effort; I tried harder and longer than necessary.

But when I was losing myself and questioned everything about the process, I needed to reconnect with my purpose for continued sanity, and I discovered that the source of all the ancient Eastern wisdom I could ever want worked right next to me in the lab as a postdoc: my brother from an Asian mother, Shengyuan Ding. He taught me and we learned together how to care for relationships more than ideas; this may be the most lasting lesson I learn from my work on this project. It will be many years in the future and in very different contexts when I realize the myriad ways he enlightened our conversations and the full impact he had on my development, even more on my emotional maturation than as a scientist. 


\begin{abstract}
L-3,4 dihidroxyphenylalanine (1-DOPA) strongly stimulates motor activity in parkinsonian patients and animal models of Parkinson's disease. Severe striatal dopamine (DA) loss characterizes Parkinson's disease and its animal models. Given the canonical rate model of Parkinson's Disease pathophysiology based on differences in DA pharmacology manifesting as electrophysiological differences in striatal projection neuron (SPN) spike rates, SPNs should increase spiking during the motor response to 1DOPA. In fact, stimulating specific subsets of these neurons to spike in freely-moving wild type and parkinsonian animals causes or inhibits motor activity as predicted. However, pharmacological effects of DA deficiency, let alone those of DA replacement, on SPN spiking activity in freely-moving animals are poorly studied and ultimately unknown. Showing the activity of SPNs of both in-/direct pathways may help elucidate mechanisms by which 1-DOPA increases motor activity to normal and sometimes abnormal levels; such mechanistic information would advance understanding about how DA is such a potent motor stimulant.
\end{abstract}

To this end, I devised a Top-hat u-array (with microdrive) for recording in the striatum while stimulating the reticulated substantia nigra. Using my micro-array, I tested 1-DOPA's acute effect on SPN spiking activity within contexts that varied in DA deficiency according to the Pitx3Null mouse's Parkinson's-like gradient of striatal DA denervation. Evidently, chronic DA denervation renders SPNs hyper-responsive to 1DOPA and a D1 agonist, SKF 81297, as indicated by exaggerated SPN spike rate responses biased by low baselines in Pitx3Null mice compared to wild-type mice. However, this may be a motor network effect on spiking as it was found in both dorsal (DA-denervated) and non-dorsal (having residual DA) Pitx3Null striatal regions. Furthermore, antidromically identifying dorsal SPNs allowed us to putatively distinguish a particularly relevant subset (striatonigral, D1-SPNs or d[irect]SPNs) known to elicit movement; serendipitously we also identified putative fibers of passage that strongly resembled striatal interneurons. D1-SPNs in Pitx3Null animals had baselines about an order of magnitude significantly below those in wild-type, and all increased firing more so in Pitx3Null than wild-type mice after drug injections, which lends some credence to the hypothesis that direct pathway SPNs are hyper-responsive during 1-DOPA-induced normal and abnormal motor behavior secondary to DA depletion. Furthermore, they uncover a need to incorporate more neural factors in explaining electrophysiological effects attributable to DA denervation and restoration pharmacology. The latter data (putative fibers) tempt the interpretation that cortical axons of passage are being mistaken for striatal fast-spiking interneurons in the literature more often than not. 


\section{TABLE OF CONTENTS}

CHAPTER 1. INTRODUCTION .1

Aim 1: Chart the Effect of DA Agonists on MSN Firing Rates over Varying Levels of DA Deficiency.. 1

Aim 2: Chart the Effect of DA Agonists on Identified Striatonigral SPNs over Varying Levels of DA Deficiency

Aim 3. To Get Lucky and Find a Random Phenomenon That Upends My

Understanding of FSI Identification

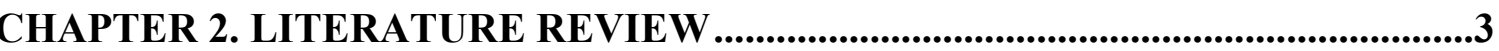

Rodent-Based Research of Parkinson's Disease .......................................................

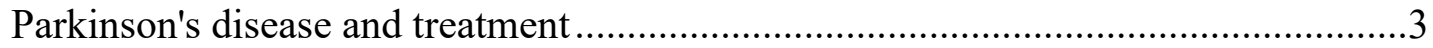

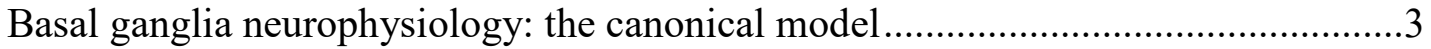

SPN neurophysiology in DA denervated conditions ..........................................

Mistaking Fibers of Passage for Fast-Spiking Striatal Interneurons .............................

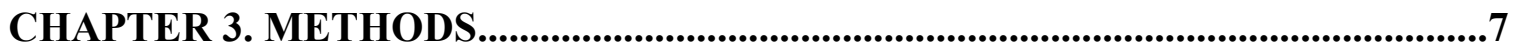

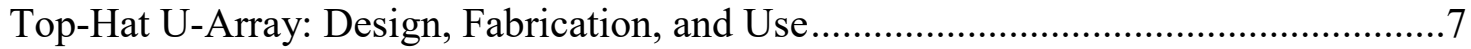

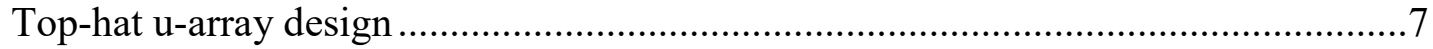

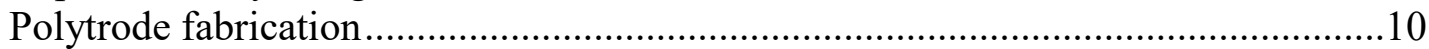

Top-hat $\mathrm{u}$-array fabrication ............................................................................... 12

Registering the skull base to the connector (interface board) and feet ................ 12

Drilling out and fitting microdrive components ............................................ 14

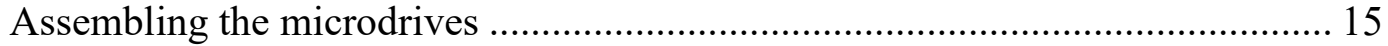

Anchoring the connector interface board and connecting polytrodes ................. 16

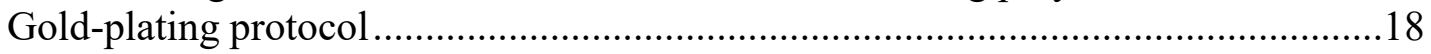

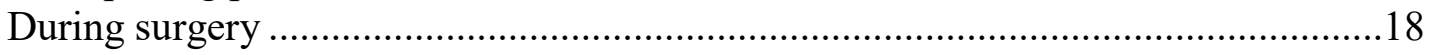

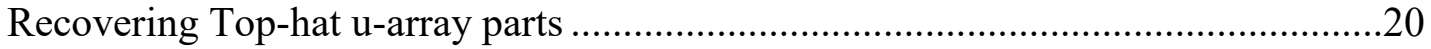

Pitx3Null Mouse Model.........................................................................................20

Recording Animal Motor Behavior before and after DA Agonism ...........................22

Electrophysiological Recordings ...........................................................................22

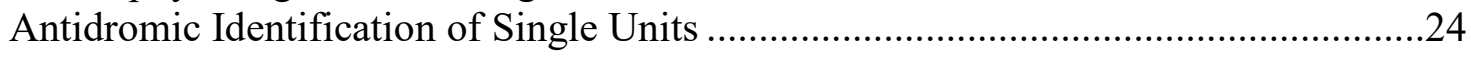

A Note on Technical Differences Between the Two Studies .....................................30

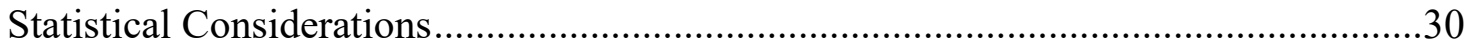

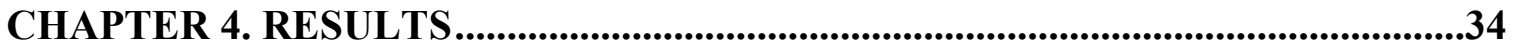

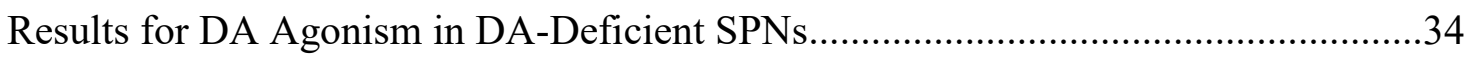

Basal SPN mean firing in WT and DA-deficient Pitx3Null mice .............................34

Response polarity differences among WT and DA-deficient Pitx3Null mice...........34

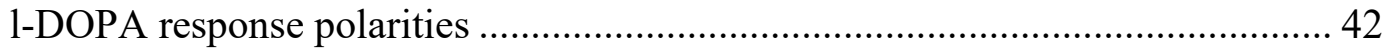

D1 agonist ersponse polarities. .................................................................. 42

Response intensity differences among WT and DA-deficient Pitx3Null mice .........44

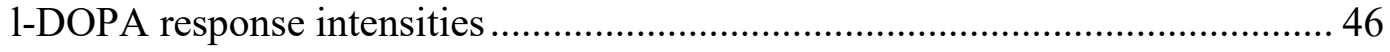




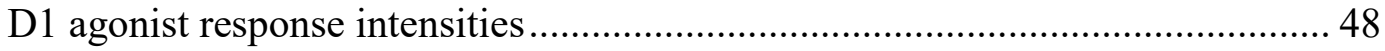

Antidromically identified SPNs are deficient in baseline spiking and respond strongly to DA agonism

Results for Fibers of Passage Mistaken as FSIs ........................................................50

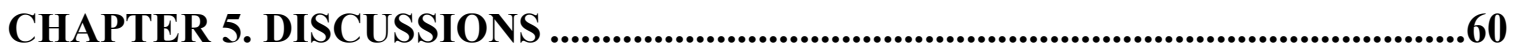

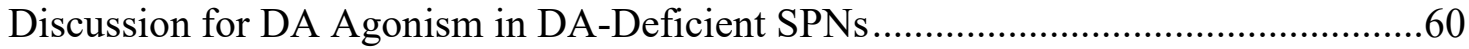

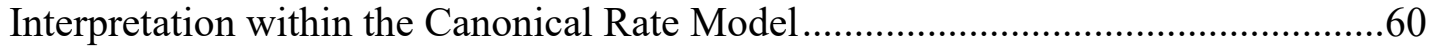

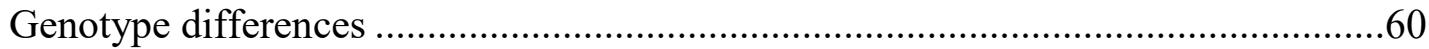

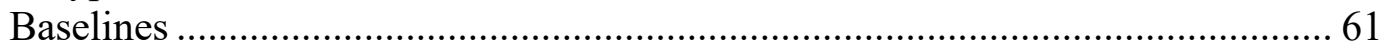

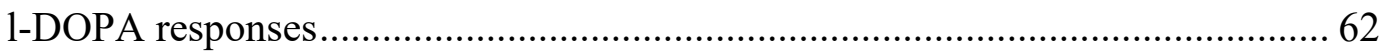

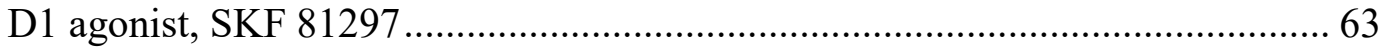

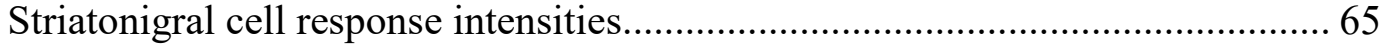

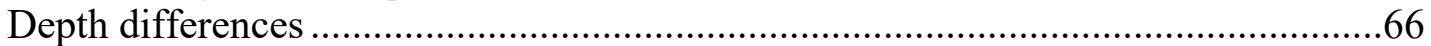

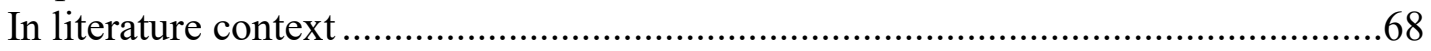

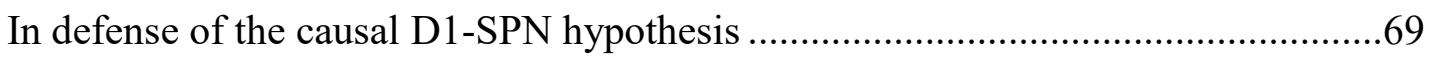

Discussion of Striatal Fibers of Passage Being Mistaken for FSIs............................. 74

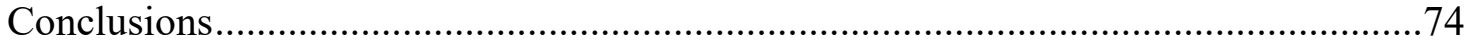

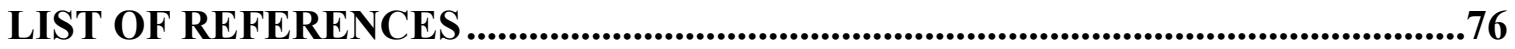

VITA 


\section{LIST OF TABLES}

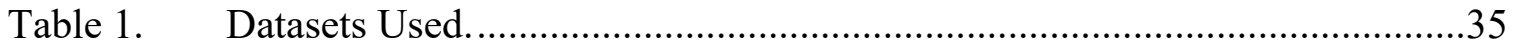

Table 2. Antidromically Identified Cell Subset. ......................................................35

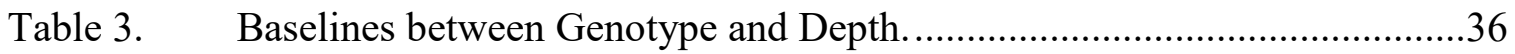

Table 4. Overall Significant Results and Post-Hoc Comparisons for Both Drug Response Datasets...............................................................................43

Table 5. Antidromically Identified Cell Baselines Between Genotype and Depth. ...49 


\section{LIST OF FIGURES}

Figure 1 Implanted Top-Hat U-Array. ……………...........................................

Figure 2. Feet Interface Microdrives with Base ........................................................

Figure 3. Capture and Spinning of Polytrode Wires.................................................... 11

Figure 4. Materials Useful in Top-Hat U-Array Fabrication. .......................................13

Figure 5. Pinning Electrode Wires to Interface Board. .................................................17

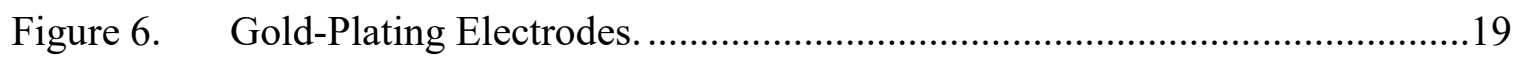

Figure 7. Recovered Top-Hat U-Array Used for Parameter Checks after Months of

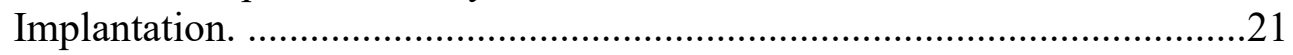

Figure 8. The Open Field Cage. .........................................................................23

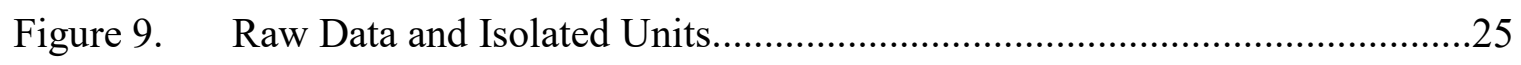

Figure 10. Exemplary Dorsal WT Data under 1-DOPA..................................................26

Figure 11. Exemplary Non-Dorsal WT Data under 1-DOPA.........................................27

Figure 12. Exemplary Non-Dorsal Pitx3 Null Data under 1-DOPA................................28

Figure 13. Exemplary Dorsal Pitx3Null Data under D1 Agonist...................................29

Figure 14. Antidromic Spike Technical Theory ...........................................................31

Figure 15. Representative Antidromic Identification Criteria and Collisions.................32

Figure 16. Modelled Mean Baselines +/- Standard Errors for Each Group....................36

Figure 17. Unexpected Responses to Selective D1 Agonism........................................37

Figure 18. Exemplary Dorsal Pitx3Null Responses to 1-DOPA. .....................................38

Figure 19. Exemplary Non-Dorsal Pitx3Null Responses to 1-DOPA.............................39

Figure 20. Exemplary Dorsal WT Responses to D1 Ag. ..............................................40

Figure 21. Exemplary Non-Dorsal WT Responses to 1-DOPA.........................................41

Figure 22. Response Polarity vs. Log Baseline..........................................................45

Figure 23. Mean Post-Drug Responses Are as Log-Distributed as the Baselines. ........47 
Figure 24. Baseline Data (Least-Squared Means) for Putative D1 Cells.

Figure 25. Pitx3Null Striatonigral Cells All Hyper-Responsive to Drug Compared

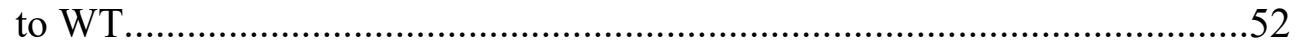

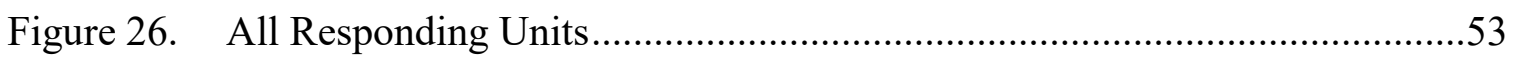

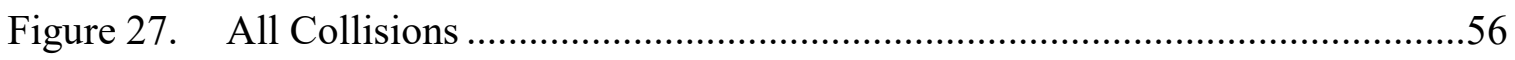

Figure 28. All Neighboring Units and Two Co-responding SPNs.............................58 


\section{LIST OF ABBREVIATIONS}

BG

D1-SPN

D2-SPN

DA

FSI

GP

ISI

1-DOPA

PD

$\mathrm{SNc}$

$\mathrm{SNr}$ basal ganglia

D1 receptor-expressing, striatonigral, striatal projection neuron D2 receptor-expressing, striatopallidal, striatal projection neuron dopamine

fast-spiking interneuron

globus pallidus

Inter-stimulus/-spike interval

1-3, 4 dihydroxyphenylalanine

Parkinson's Disease

substantia nigra compacta

substantia nigra reticulata 


\section{CHAPTER 1. INTRODUCTION}

\section{Aim 1: Chart the Effect of DA Agonists on MSN Firing Rates over Varying Levels of DA Deficiency}

In order to contrast the spiking effects of DA agonists in the context of DA deficiency against those of WT mice and against the non-dorsal Pitx3Null (DAsufficient) context, we will use extracellular single-neuron recording during freelymoving behavior.

- Aim 1a. To contrast l-DOPA's effect on dorsal SPN spiking in Pitx3Null mice against that of WT during free behavior. Preliminary data support expectations.

- Aim 1b. To contrast the effect of D1 agonist, SKF 81297, on dorsal SPN spiking in Pitx3Null mice against that of WT during free behavior. D1 agonism also accompanies increased normal and abnormal motor activity in this and other models of DA responsiveness in the DA deficient context (Li\&Zhou, 2013; Bishop et al, 2005.)

- Aim 1c. To correlate SPN rate-increases with dorsoventral recording depth to show an effect of DA deficiency on SPN spike rates.

\section{Aim 2: Chart the Effect of DA Agonists on Identified Striatonigral SPNs over Varying Levels of DA Deficiency}

In an effort to show that most cells increasing firing during movement are indeed D1-SPNs, we will perform separate recordings mirroring those above and afterward identify putative D1-SPNs. Since D2-SPNs do not have excitable fibers in the substantia nigra reticulata, spikes antidromically elicited from that region putatively identify D1SPNs as striatonigral.

- Aim 2a. To contrast 1-DOPA's effect on dorsal D1-SPN spiking in Pitx3Null mice against WT during free behavior.

- Aim 2b. To contrast D1agonist's effect on dorsal D1-SPN spiking in Pitx3Null mice against WT during free behavior.

\section{Aim 3. To Get Lucky and Find a Random Phenomenon That Upends My Understanding of FSI Identification}

Striatal FSIs are routinely identified by their relatively high rate and narrow waveshape; however, the serendipitous discovery of their antidromic spiking evidence indicates that they might be fibers of passage. 
- Aim 3a. To collate antidromic evidence for recording striatal fibers of passage; characterize said waveforms and their corresponding units. 


\title{
CHAPTER 2. LITERATURE REVIEW
}

\author{
Rodent-Based Research of Parkinson's Disease
}

\section{Parkinson's disease and treatment}

At autopsy, Parkinson's Disease (PD) patients show an especially marked degeneration of the nigrostriatal fibers that supply the striatum with dopamine (DA). (Kish et al. 1987 or 1988; Hornykiewicz 2001; Kowdoer et al. 2013 ). This otherwise intense striatal DA innervation (from the compact substantia nigra) complements heavy striatal DA receptor expression (Levey et al 1993; Yung et al 1995). Striatal DA loss invariably precipitates hypokinesia once it reaches $\sim 80 \%$ (Kim et al., 2000 see also Fig. 4 from Schwarting and Huston, 1996) and is proposed to induce a hypersensitivity to dopamine (Cenci \& Konradi, 2010). After DA depletion, DA agonism elicits cellular markers (e.g. ERK, delta/FosB) of supersensitive dopamine D1 receptor stimulation (Gerfen et al, 2002; Pavon et al, 2006; Corvol et al, 2004) as it stimulates increases in both ab-/normal motor activity (Francardo \& Cenci, 2014; Li et al, 2015).

The most common PD treatment is DA replacement therapy using 1-3,4dihydroxyphenylalanine (I-DOPA) because of its efficacy in stimulating motor activity (Olanow et al, 2009). In addition to normal motor activity, it may eventually induce hyperkinetic side effects, known as 1-DOPA-induced dyskinesias (Olanow et al, 2009, Bastide et al., 2015). During 1-DOPA's effect on behavior, it affects DA deficient striatal information processing, but this is poorly characterized (Iversen, 2010, pg 438), which is one striking reason for a continued focus on the behavioral and physiological manifestations of DA pharmacology in the context of DA loss (Olanow et al, 2009).

\section{Basal ganglia neurophysiology: the canonical model}

The striatum, a key component of the basal ganglia (BG) motor control circuit, is thought to be directly responsible for the motor symptoms of PD and of PD treatment (Hornykiewicz 2001; Franco \& Turner 2012). The striatum is the BG structure that processes the majority of the BG's synaptic input, which is sent downstream via two distinct output pathways. One is the direct pathway in which dopamine D1 receptorexpressing striatal projection neurons (D1-SPN, a.k.a. D1 striatal spiny projection neuron, D1-SSPN, D1 medium spiny neuron, D1-MSN, and dSPN) can ultimately activate movement via spiking. The other is the indirect pathway in which dopamine D2 receptor-expressing SPNs (D2-SPN, etc, and iSPN) can ultimately inhibit movement via spiking (Kravitz et al, 2010). These downstream effects of spiking on behavior operate in both intact and DA-lesioned mice (Kravitz et al, 2010).

Mechanistically, these phenomena are interpreted most often via the canonical rate model of the BG explained thus: both cell types release GABA but have opposing 
effects due to the nuclei into which they synapse. Direct pathway SPN spikes disinhibit thalamocortical motor networks (allowing behavior) by directly inhibiting BG output nuclei, e.g. the substantia nigra reticuluata (SNr) (Kravitz et al, 2010; Freeze et al. 2013). Conversely, indirect pathway SPNs indirectly inhibit thalamocortical motor networks, by synapsing on intermediate inhibitory BG nodes; synapsing into these nodes reverses the polarity of their effect on downstream neuronal, and ultimately behavioral, activity. By way of example, specific D2-SPN activation has been shown to cease movements (Kravitz et al, 2010, Sano et al.,2013); this is interpreted canonically as inhibiting an intermediate inhibitory nucleus, which disinhibits the (inhibitory) SNr ultimately rendering thalamocortical motor nuclei further inhibited and reducing movement. Overall then, increases in D1-SPN spiking promote movement via the direct pathway, and increases in D2-SPN spiking inhibit it via the indirect pathway.

\section{SPN neurophysiology in DA denervated conditions}

Understanding what role stimulation of DA receptors on SPNs plays in their spiking might reveal mechanisms underlying 1-DOPA's cell-level effects that accompany motor activity and could have a bearing on the interpretation of DA's function in the normal and pathological BG. Published results are interpreted to affect spiking indirectly through networks and/or are inconsistent. For example, in vitro SPN recordings from DA-lesioned animals are generally interpreted in terms of effects on corticostriatal glutamate release (reduced by aberrant D2 signaling after DA denervation and rescued by l-DOPA; Picconi et al, 2004) and long-term synaptic changes (aberrant $\mathrm{Ca}^{2+}$ currents after DA depletion; Prieto et al, 2009), whereas the canonical model is more directly invoked to explain in vivo anesthetized results in terms of spike output (increased background spiking after DA depletion normalized by 1-DOPA; Kita\&Kita, 2011a, b). However, the fact that anesthetized (and in vitro) SPNs show drastically different spontaneous firing properties and membrane potential states than in awake animals (namely, less variable rates and bimodal resting membrane voltage; Mahon et al, 2006; Kish et al, 1999; Rebec, 1998) highlights the importance of behaving animal recordings in exploring the effects of DA denervation on SPN spiking during behavior.

Despite the seemingly straightforward connection between direct stimulation of D1-SPNs causing their spiking and consequent motor activity (Kravitz et al, 2010) and supersensitive DA receptors (which SPNs likely express more heavily than any other brain area due to their baseline expression being many-fold higher; see e.g. Fig. 2 of Delis et al., 2013) possibly being one cause of LID (Cenci \& Konradi, 2010), effects of DA agonism on DA-deficient SPN spiking during such behavior is poorly researched. Such recordings are rare in primates (not one according to the following review: Boraud et al., 2002, at least one thereafter: Liang et al., 2008, Singh et al., 2015, 2016) and rare and conflicting in rodents (Iversen, 2010, pg. 438; Kish et al, 1999; Chen et al, 2001). Nonetheless, knowing these receptor and cell level events is necessary if one wants a full account of BG functionality to explain 1-DOPA's behavioral effects in enough mechanistic detail to refine understanding of how SPN activity informs behavior. 
Since specifically D1-SPN rate increases induce motor behavior (in both DA un/lesioned conditions; Kravitz et al, 2010), and DA denervation of striatal cells sensitizes the BG to do the same under 1-DOPA (thus DA) agonism (Corvol et al, 2004; Cenci and Konradi, 2010), then it is expected that at least part of the underlying mechanism of motor activity induction by 1-DOPA would be the rendering of D1-SPNs hyperresponsive to DA agonism by DA denervation (Feyder et al., 2011, Iversen, 2010, pg. 438, and Murer \& Moratalla, 2011).

To this end, we recorded SPN responses to 1-DOPA and D1 agonist, SKF-81297 in DA deficient (Pitx3Null dorsal striatum, defined further in Methods) and DA sufficient (Pitx3Null non-dorsal and WT dorsal striata) conditions. We further identified a subset of striatonigral SPNs antidromically in order to begin interpreting the results from the in/direct pathways perspective.

The Pitx3Null mouse models 1-DOPA-induced motor activity. This is because it has a chronic, spatially restricted, dorsoventral DA deficiency gradient and a robust ab/normal motor response to l-DOPA that is absent in WT animals (Francardo \& Cenci, 2014; Hwang et al, 2005). Contrasting these against WT mice, we looked for electrophysiological evidence consistent with the predominant hypothesis (see e.g. Cenci and Konradi, 2010 and Murer \& Moratalla, 2011) that l-DOPA induces motor activity via rate increases in D1-SPNs. By testing SPN responses to DA agonism, we expected to discover a spiking mechanism putatively underlying LIM. Specifically, we looked for any evidence that DA deficiency would be associated with a change in baseline rate between groups before we tested the hypothesis that DA-denervated SPNs (i.e. from dorsal Pitx3Null mice striata) show exaggerated spiking responses during their DAagonism-induced motor activity superseding those of dorsal WT SPNs (fully DA innervated). In addition, we tested the hypothesis that dorsal Pitx3Null SPNs increased spiking in response to DA agonism more so than non-dorsal Pitx3Null (less DA denervated) SPNs. Finally, we re-tested the first hypothesis in a subset of antidromically identified SPNs.

\section{Mistaking Fibers of Passage for Fast-Spiking Striatal Interneurons}

Electrophysiologically recording the striatal neurons of rodents is a commonly performed technique in the neuroscience literature concerning normal and diseased brain function (e.g. Parkinson's disease, drugs of abuse). (Gittis et al, 2011, Kubota et al, 2009, Lee et al, 2017, o'Hare et al, 2017, Sagot \& Zhou, submitted) Aside from the inherent technical difficulties of electrophysiological recordings, focusing research questions on a particular subtype of neuron further decreases the likelihood of sampling from a population of interest. According to many publications (Graveland \& Difiglia 1985, Saka 2002, Rymar et al 2004), interneurons represent $<5 \%$ of total striatal neurons, which can further be broken down into different interneuron types; e.g. tonically active neurons (TANs), and fast-spiking interneurons (FSIs), also known as parvalbumin-positive (PV+) interneurons. Luk and Sadikot, 2001, report that PV+ interneurons make up only $0.7 \%$ of striatal interneurons. Thus attempts to record these would at least initially be expected to 
have much fewer opportunities to do so and be reflected in unbiased samples at approximately the same rate.

However, in vivo electrophysiologists have reported FSI proportions in striatal samples to account for 7-30\%. (Barnes 2005, Kubota et al, 2009, Hernandez et al, 2013, Berke et al 2004 and 2008, Gage et al, 2010, Schmitzer Torbert and Redish 2009, Lee et al, 2017) When unintentional (as opposed to e.g. Mallet, 2005), this obvious discrepancy has been hypothesized to result from their biased distribution towards the dorsolateral striatum (Berke 2011) and a general facility for recording data from neurons having larger somata and higher spike rates due to their more obvious presence during spike sampling before recording. (Heinricher in Microelectrode Recording in Movement Disorder Surgery, 2004 ed.)

Identification metrics in these cases have used primarily waveshape, and some combination of firing rates, ISI histograms, autocorrelograms, or peri-event rasters (Barnes et al, 2005, Mallet 2005 and 2006, Kubota 2009, Schmitzer Torbert and Redish 2009, Tepper, 2010, Gittis et al, 2011, o'Hare et al, 2017), but these criteria are inconsistently seen, reflecting the lack of knowledge over clearly distinguishing FSI traits in vivo; unambiguous identification is rare (Tepper and Koos, 2017). Thus resolving the identity of these units potentially stands to shed new light on previous conclusions, e.g. the task-relevance of FSIs (Berke et al., 2004, Kubota et al, 2009, Tepper \& Koos, 2017)

Some hint of their identity came accidentally when, during antidromic identification of direct pathway SPNs, I recorded antidromic responses in the striatum having the waveshapes and ISI characteristics much more commonly associated with FSIs. Other evidence supports this interpretation: response latencies predicted by theoretical expectations of myelinated axons (Jinnai \& Matsuda, 1979) and putative fibers clustered with up to 4 others (as reported: Berke, 2008) These tempt the interpretation that these are better explained as cortical fibers of passage, however more research should confirm this hypothesis. 


\section{CHAPTER 3. METHODS}

\section{Top-Hat U-Array: Design, Fabrication, and Use}

In order to record neural activity from freely moving animals, a device must effectively host the interface between the brain and the recording equipment, must minimize interference with the animal's behavior (most likely being lightweight, $\sim 10 \%$ of body weight) and be able to withstand months of implantation on the animal's head, and -if the neural coverage is not sufficient- must reposition electrodes in order to optimize recordings as well. Usually this device is referred to as a microarray, with or without a microdrive for repositioning electrodes.

In order to accomplish these using as few and inexpensive resources as possible, I designed a microarray I call the "Top-hat u-array' due to its appearance on the animal once implanted. (Figure 1) The design, fabrication, and use of this microarray are described here.

\section{Top-hat u-array design}

In designing the array, considering the brain areas from which one will record or stimulate forces the device to have certain parameters since volume and component proximity are limited; in our case, the simultaneous recording/stimulation (using electrode sets inside stabilizing tubes having $<0.26 \mathrm{~mm}$. outer diameter) from a maximum of four locations having inter-site spacing $>0.5 \mathrm{~mm}$. were made possible in the rostral cortex, striatum (str), and substantia nigra pars reticulata $(\mathrm{SNr})$ of one hemisphere of a mouse. Flexibility of the design for starting materials is maintained by using small -compared to the entire available volume-, modular pieces (e.g. tubes, screws, springs, etc. as opposed to 3D printed parts). Flexibility for accommodating other brain areas and hemispheres is maintained by pre-registering the top hat's components with respect to one another and ultimately to the skull-, i.e. 1:1 placement of array components into a base that doubles as a "skull map" (a common frame of reference) without having to place everything relative to each other during surgery. Flexibility in the dorsoventral positioning of electrodes is maintained by including a microdrive mechanism, which in our case was done by registering another skull map on top of the first in order to subtractively form "feet" that interface between the drive screw and the carrying tube. (Figure 2 showcases a completed Top-hat minus debris shield and ground wire.)

A miniature connector and interface, or breakout, board for connecting electrodes to the input connector pins and ultimately to the recording system was in our case essential in terms of minimizing weight, facilitating input connections, and facilitating registration to the stereotax during implantation. The EIB-16 from Neuralynx we used might be unable 


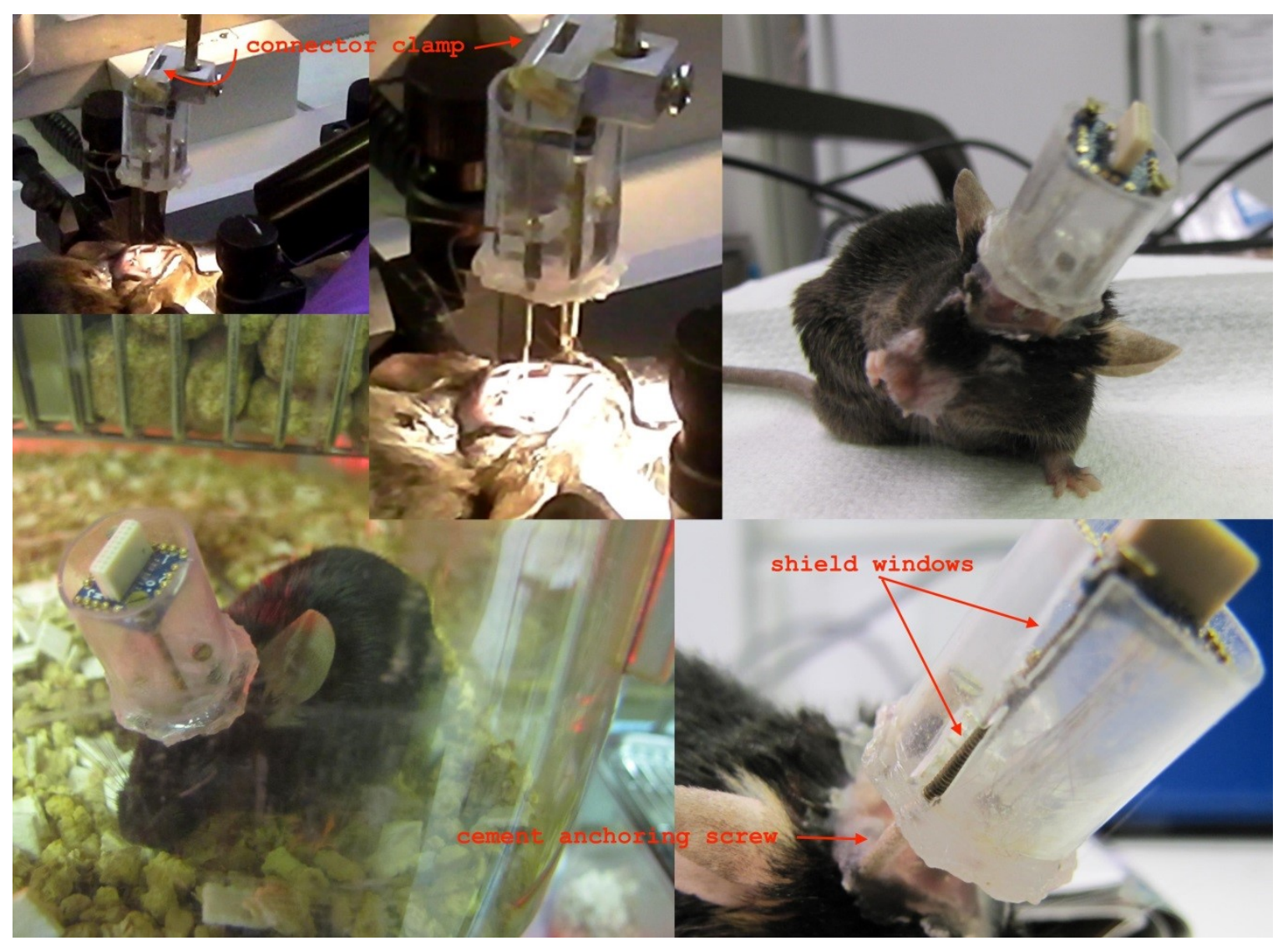

\section{Figure 1 Implanted Top-Hat U-Array.}

Upper left and upper middle - the implantation steps preceding recovery during ground wire placement. Upper right - the animal grooming its paws after recovery. Lower left the animal roaming its cage after recovery. Lower right - close-up of implant highlighting placement with respect to skull, dental cement anchoring to screws, windows in debris shield, and translucence 


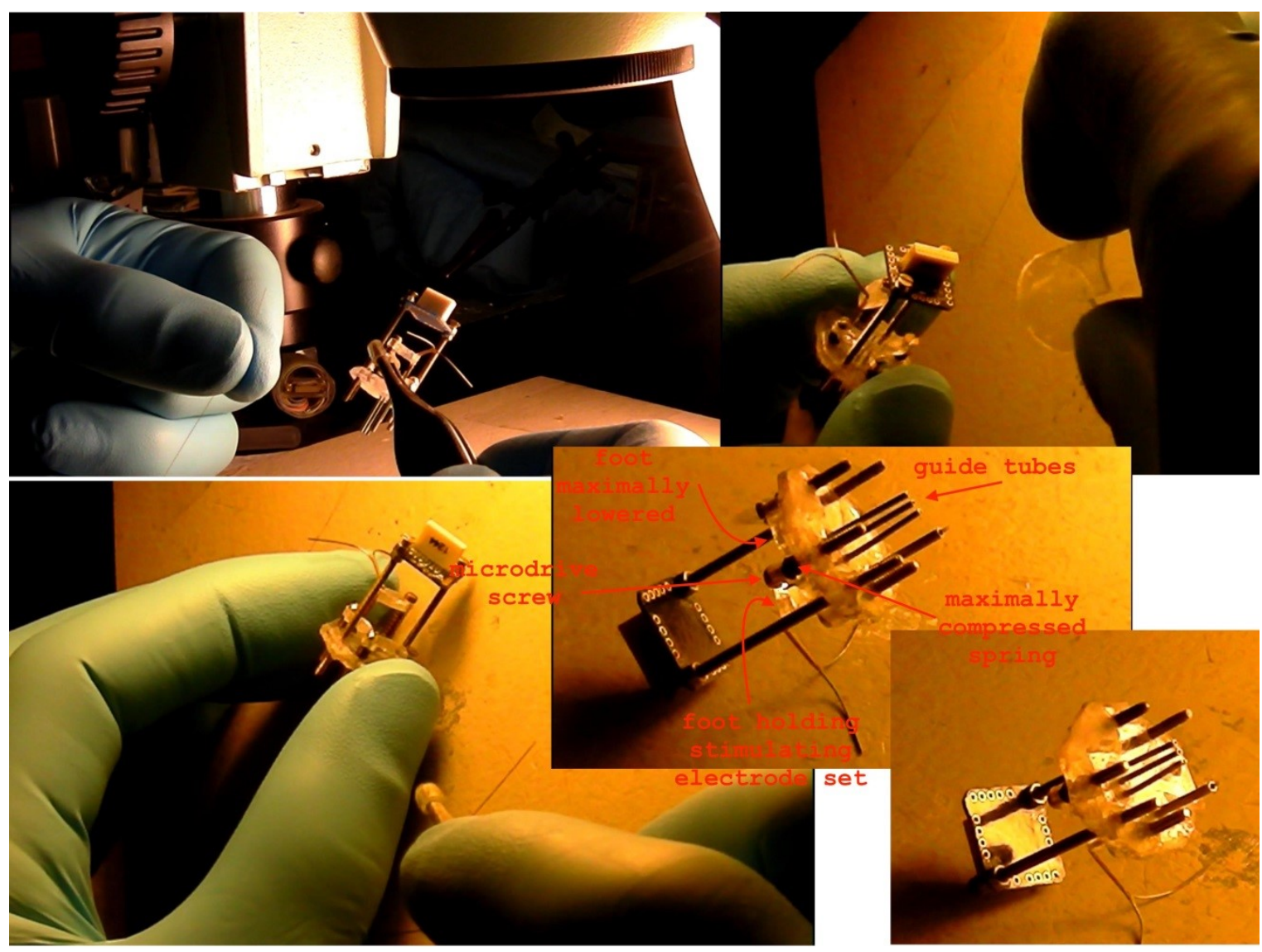

Figure 2. Feet Interface Microdrives with Base.

Upper left - an assembled microdrive set with connector anchored to base. Upper right shield tubing during placement (although this step is normally done after electrode placement, gluing, and pinning): window seen between thumb and forefinger on right. Lower left - preparing to insert polytrodes into carrier tubes. Lower middle and right close-ups of assembled microdrive set and anchored connector (stimulating electrode set clearer although its slight protrusion is problematic due to fragility of fine tips) 
to be improved upon, and in our case doubles as a cover for the Top-hat u-array, keeping most environmental debris out for longer than other arrangements. (Furthermore, they were re-usable at least once, if not multiple times until obvious defects arose or the connector did not hold its mate $>5$ minutes during animal behavior.)

Similarly, in such an arrangement, a transparent and durable shield should protect the mechanisms and wiring; in our case, clear, cylindrical, plastic tubing (metallic wire mesh may also work to a slightly lesser extent and allow for electromagnetic shielding when attached to ground) that fits snugly around the entire contraption resting atop the base is essential for excluding debris and interference from the animal's movement. However, the ability to insert tools for troubleshooting through this wall comes at the cost of excluding debris (but can facilitate rinsing if appropriately situated), so optimizing the size and placement of windows in the shield should be carefully considered. (Figure 2, upper right)

\section{Polytrode fabrication}

Polytrodes (electrodes wrapped together in various numbers) are necessary in order to complete the array; fabricating them before the microarray affords an economy of scale (provided the protocol yields acceptable work-product) since they are less labor intensive (and thus easier to make in bulk) and because having them available allows one to focus on finishing the microarray once begun.

The protocol of Liao et al, 2011, was adapted to our purposes (since we used 12.5 um nichrome wire from Kanthal Palm Coast, Palm Beach, FL); specifically starting from the proposition that 4 wires having 25 um outer diameter can withstand 60 turns/cm without failing, and assuming a linear relationship between the number of turns and the number and diameter of wires to be included in the polytrode, then twice the number of wires at half the diameter should yield the same acceptable turn ratio. An intuitive way to consider this approximation is that one could theoretically cut each 25 um wire into two 12.5 um wires without disturbing too much the turn ratio; thus, eight $\sim 12.5$ um wires should withstand about 60 turns/cm. (Figure 3)

(More electrodes than four were used per polytrode since preliminary results showed non-orthogonal -highly correlated- data on four non-splayed electrodes, i.e. overlapping data, rendering tetrode utility comparable to a single electrode. At times 8 and sometimes 6 wires were used, which helped splay some wires from the others and allowed us to use only the most orthogonal data during spike sorting. Incidentally, introducing splay manually had a similar though less reliable effect, although splaying should generally not precede insertion of the polytrode through its stabilizing or carrying tube, i.e. the final steps.) 


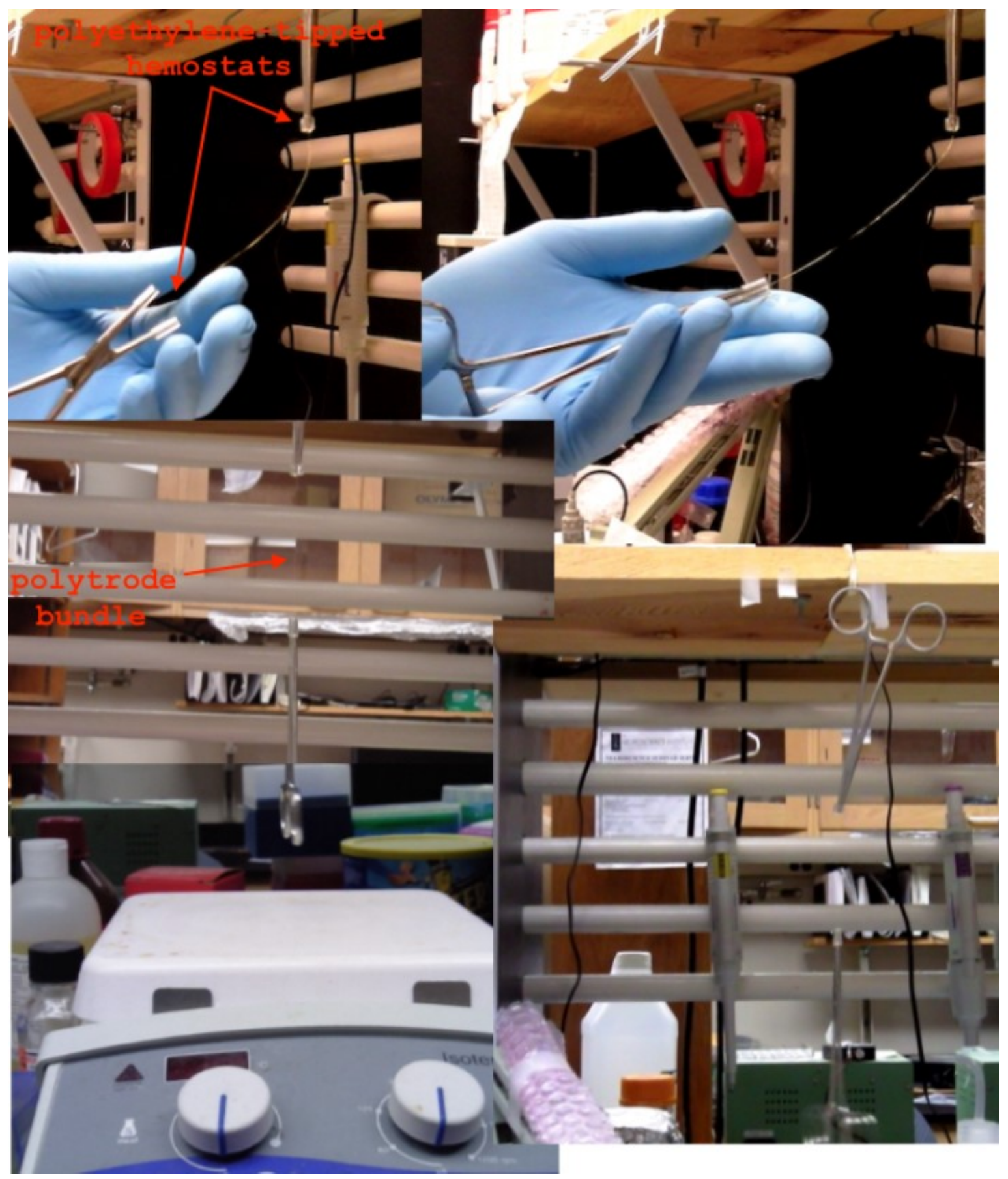

Figure 3. Capture and Spinning of Polytrode Wires.

Upper left and right - capture of wetted polytrode wires within polyethylene-tipped hemostats. Lower left - hemostats suspended above magnetic stirrer on lowest spin setting ( $\sim 1 \mathrm{rev} . / \mathrm{sec})$. Lower right - hemostats set held in polyethylene tubing loop (away from stirrer) unspinning freely until rest 
Taking the number of wires to be turned together into the polytrode was done by folding an extended piece of nichrome as many times as necessary to create the desired multiplicity at $\sim 15 \mathrm{~cm}$ length (i.e. $\sim 90 \mathrm{~cm}$ for a 6 -trode, 120 for $\mathrm{n} 8$-trode), then running the bunch under a stream of water while pinching the tip with more folds (i.e. leaving one tip loose to be straightened uniformly by the water); once wires stayed in a smooth bundle, this pinched section was transferred carefully to straight-tipped forceps to be hung by its handle on two appropriately spaced and sized knobs. Bundling the wires at the other tip may require another run under water, but once both tips are held each by straight-tipped forceps (one pair of which is hooked onto the knobs), then a magnetic stirrer is placed underneath the lower pair and centered with respect to it. The turn ratio then translates well into the rate at which the polytrode is spun: 1 minute/cm for turn ratios of $60 / \mathrm{cm}$.

Once the polytrode has the desired number of turns, the magnetic stirrer is removed and the polytrode allowed to un-spin and re-spin until coming to rest (Figure 3, lower right), at which point it is safe to remove the forceps and store the polytrode somewhere that dust and static are minimized (for ease of array fabrication).

\section{Top-hat u-array fabrication}

\section{Registering the skull base to the connector (interface board) and feet}

4.

A picture and description of parts used in the Top-hat u-array is found in Figure

(In order to facilitate fixture, before gluing, of components into the plastic, drill bit diameters should be rated for widths at least 10-15um smaller than the outer diameter of the tube expected to be placed through the resulting hole, e.g. $0.25 \mathrm{~mm}$. for a $31 \mathrm{ga}$ $(0.26 \mathrm{~mm}$ outer diameter) tube such that insertion is smooth yet temporarily held in place without gluing.)

In the first step of Top-hat u-array fabrication, two polycarbonate pieces of sufficient size to accommodate the footprint of the shielding tube $(\sim 2 \times 2 \mathrm{~cm})$ are registered to each other by means of two holes simultaneously precision drilled (MicroLux, Micro-Mark, Berkeley Heights, NJ) at opposite corners of both layers -one layer on top of the other- and inserting appropriately sized rigid tubing until flush with at least one layer of the two.

(Measuring exact locations of sites on this two-layered piece can be difficult without having either markings in the microdrill's retaining bars or preset markings on the piece itself; we used the former in order to avoid repeating the latter for every Tophat.) 


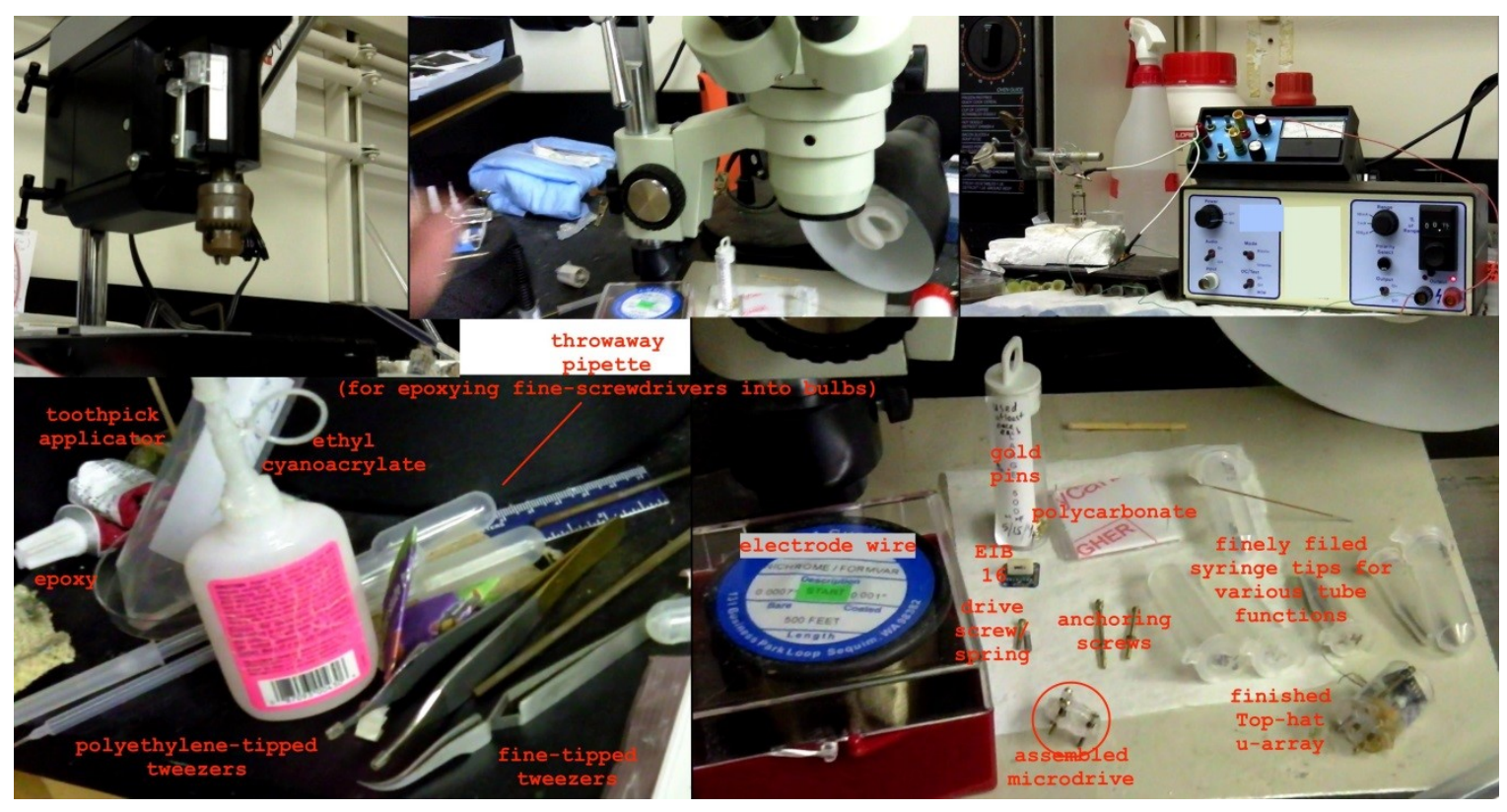

Figure 4. Materials Useful in Top-Hat U-Array Fabrication.Upper left precision drill press and guide bar (having metric notches unseen here). Upper middle dissection microscope and fabrication platform having lighting. Upper left - stimulus isolator, impedance meter, and plating station. Lower left - precision tools and consumables. Lower right - microdrive and polytrode consumables. 
Marking the anteropesterior (AP) and mediolateral (ML) locations of desired recordings is straightforward; define a center of the base (perhaps as bregma or lambda or some variant), then take each coordinate as an ordered pair $-(\mathrm{x}, \mathrm{y})$, where $\mathrm{x}=\mathrm{ML}$ or AP coordinate, and $\mathrm{y}=$ the respective other- then carefully drill through both layers of polycarbonate using the drill bit corresponding to the outer diameter of the smallest carrying tube (in our case $0.25 \mathrm{~mm}$, corresponding to the 31 ga tube). The resulting holes will eventually accommodate the carrying tube in the top layer and mark the guide tube location in the second layer.

(Using smaller drill bits before larger in the fabrication process mitigates the impact of some drilling errors.)

Without turning the piece over or separating the layers, drill eight holes appropriately sized (in our case, $\sim 1.0 \mathrm{~mm}$ ) to fit the drive screws (in our case, 00-90 screws, $1.194 \mathrm{~mm}$ outer diameter) in an equilateral octagon around the center of the base, 4 of which will be covered by interface board corners -2 of those being used for screwing the board to the base- and 4 of which will anchor the carrying tubes to the base and act as an actuator for the microdrive mechanism. The distance from the center to the screw holes should equal half the distance between the two screws that will anchor the interface board (in our case the two screw holes at opposite corners of the EIB-16, which have a center to center distance $\sim 12.5 \mathrm{~mm}$ ).

\section{Drilling out and fitting microdrive components}

Separate the layers; choose a top layer -for making feet that connect the carrying tube to the drive screws- and a bottom layer -as the base to hold guide tubes, anchoring screws, drive screws, and the shielding tube.

The next largest dill bit (in our case, sufficient to snugly fit a 26 ga guide tube, $\sim 0.45 \mathrm{~mm}$, for polytrodes and to fit a 20 ga for the stimulating electrode guide tube, $\sim 0.9$ $\mathrm{mm}$ ) is used to drill out the marked guide tube sites on the chosen bottom (base) layer. Drilling an extra hole within the perimeter will allow a ground wire (otherwise passed down around the edge of the base) to be passed directly through the base and provide another anchor point for dental cement during implantation. Guide tubes may then be glued having the desired protrusion from the base being careful to ensure that they are as parallel as possible to each other.

On the same layer, a tap for the drive screws is used to carve into the 8 perimeter holes. These screws should be long enough $(\sim 25 \mathrm{~mm}$ in our case) to protrude from the bottom of the base (by $\sim 6-8 \mathrm{~mm}$ in our case) in order to provide anchoring to the skull using dental cement. Although not all 8 need be used (accommodating up to 4 drive screws +2 anchoring screws + optional other anchoring screw projections from base), whichever are used varies primarily according to the ease with which certain feet connect to the carrying tubes (in our case, the EIB connector orientation was necessarily also 
considered). The final step on the base layer is to align guide tubes with respect to each other.

As for the top layer, the primary concern is ensuring that whichever 4 (or fewer) of the 8 screw holes used to accommodate the drive screws, which hold the carrying tube feet, are designed and then fabricated without error; minimizing the distance between the drive screw and its carrying tube will ultimately maximize the downward force of the screw on the carrying tube, minimizing the chance to get stuck. Since it is much easier to drill out each drive screw hole than form the feet, the largest drill bit (in our case, 1.25 $\mathrm{mm}$ ) is used to drill out all 8 perimeter holes, and 4 (or fewer) feet are then designed by marking boundaries between them -e.g. using a fine-tipped felt pen or scalpel tip- then formed subtractively -i.e. by removing surrounding shards of plastic at first using diagonal pliers then by a razor under a dissecting microscope. It is critical here to carefully remove as much plastic as possible from around the carrying tube hole (in order to keep it from interfering with others during use on the animal) without risking the integrity of the hole. The area around the drive screw holes requires similar care since they may otherwise interfere with shield tube placement at the final step.

(In either case, when a tube hole is compromised, superglue may carefully be applied either shortly thereafter or in the next step during the process of positioning carrying tubes inside guide tubes and affixing them to the feet, although be warned that superglue in such a confined space can easily cause more harm than good in such a delicate situation.)

\section{Assembling the microdrives}

Assembling the feet (once they are approximately shaped enough to be assembled) into the drive mechanisms can minimize damage to the feet during the final touches of their formation since they otherwise become much more difficult to hold once removed from one another. In order to assemble the feet, thread a drive screw (in our case, 3/8" 00-90 screw) downward through the foot's drive screw hole and into a spring. Then using a fingertip to steady this combination on the tip of a screwdriver (approximately upside-down), begin screwing this screw into the drive screw hole on the base, i.e. that has been tapped. Perform the same for the remaining feet and drive screws/springs unless they interfere with each other enough to preclude removing further shards. (Figure 4, lower right, circled)

Once the base holds all the microdrives, remove shards from the feet until they no longer interfere with each other upon use; inserting the carrying tubes through their holes into the guide tubes and screwing the drive screws as far as possible into the base can hold the feet in place to facilitate such removal.

(Replacing gloves when handling the fine gauge tubes minimizes the chance that debris will block the tube during polytrode or stimulating electrode insertion.) 
Once the carrying tubes have been inserted into the guide tubes through their foot holes, they should be checked for patency using thin wire (in our case $\sim 0.05-0.1 \mathrm{~mm}$ ), and then glued to the feet while ensuring that their maximum extension and retraction do not interfere with the interface board or protrude too much. (In our case, guide tube patency was checked before precisely inserting the stimulating electrode set itself into the foot under the dissection microscope; carrying tubes were pre-pared in our case at $15 \mathrm{~mm}$ long to fit $10 \mathrm{~mm}$ long guide tubes and a presumed $17 \mathrm{~mm}$ EIB height) Ensure that at maximum extension the carrying tube does not exit the guide tube longer than $\sim 0.3-0.5$ $\mathrm{mm}$, the approximate depth of dura from the top of the skull. Applying some superglue around each carrying tube on top of its foot mitigates the risk that it will slide within its hole during drive screw retraction, but doing the same underneath the foot risks fastening the carrying tube to the guide tube, so this latter step is best done with the drive screw fully retracted.

\section{Anchoring the connector interface board and connecting polytrodes}

Next, the connector interface board can be anchored to the base. First threading the anchoring screw (in our case, 1" 00-90 screw, Fasteners and Metal Products Corp., Waltham, MA) through a nut, then the board, then another nut allows one to tighten the nuts around the board so that movement of the board in its final situation is minimized. Once this is done for one side of the board, that anchoring screw is threaded into its tapped hole in the base.

Threading the second screw begins the same as the first, but the lower nut should only be tightened after this screw has been threaded into its anchoring hole (otherwise the screw might not tighten into the base but against the nut); serrated tweezers around the nut and a screwdriver to hold the screw in place are helpful here. More important in this step is the final height of interface board on each screw, which should be an equal distance from the base on both anchor points in order to minimize torsion on the connector board.

Polytrodes are then inserted into their carrying tubes (or stabilizing tubes first if necessary: in our case polyimide tubing, Part No. MIL 38, Microprobes, Gaithersberg, MD) and extended sufficiently from the guide tube in order to allow $\sim 1 \mathrm{~mm}$ of the entire tip to be splayed before being dipped in ethyl cyanocrylate (Jog et al, 2002) and dried for the final cut exposing the electrode wires uniformly. Finally, taking the guide tube tip as skull depth, the cut polytrode is lowered as far as possible in order to define the furthest ventral location from which to be recorded unless the highest point to be recorded is more critical. In either case, the carrying tube is not yet glued to its contents -nor, ideally, are the stabilizing tubes glued to theirs- before connecting all individual electrode wires (except ground and reference) to the connector board and checking for connectivity during gold-plating or waiting until gold-plating is complete. At this point the electrode wires are individually pinned to the interface board; a gentle tug on each wire after pinning ensures that it has not broken at the point of contact. (Figure 5) 


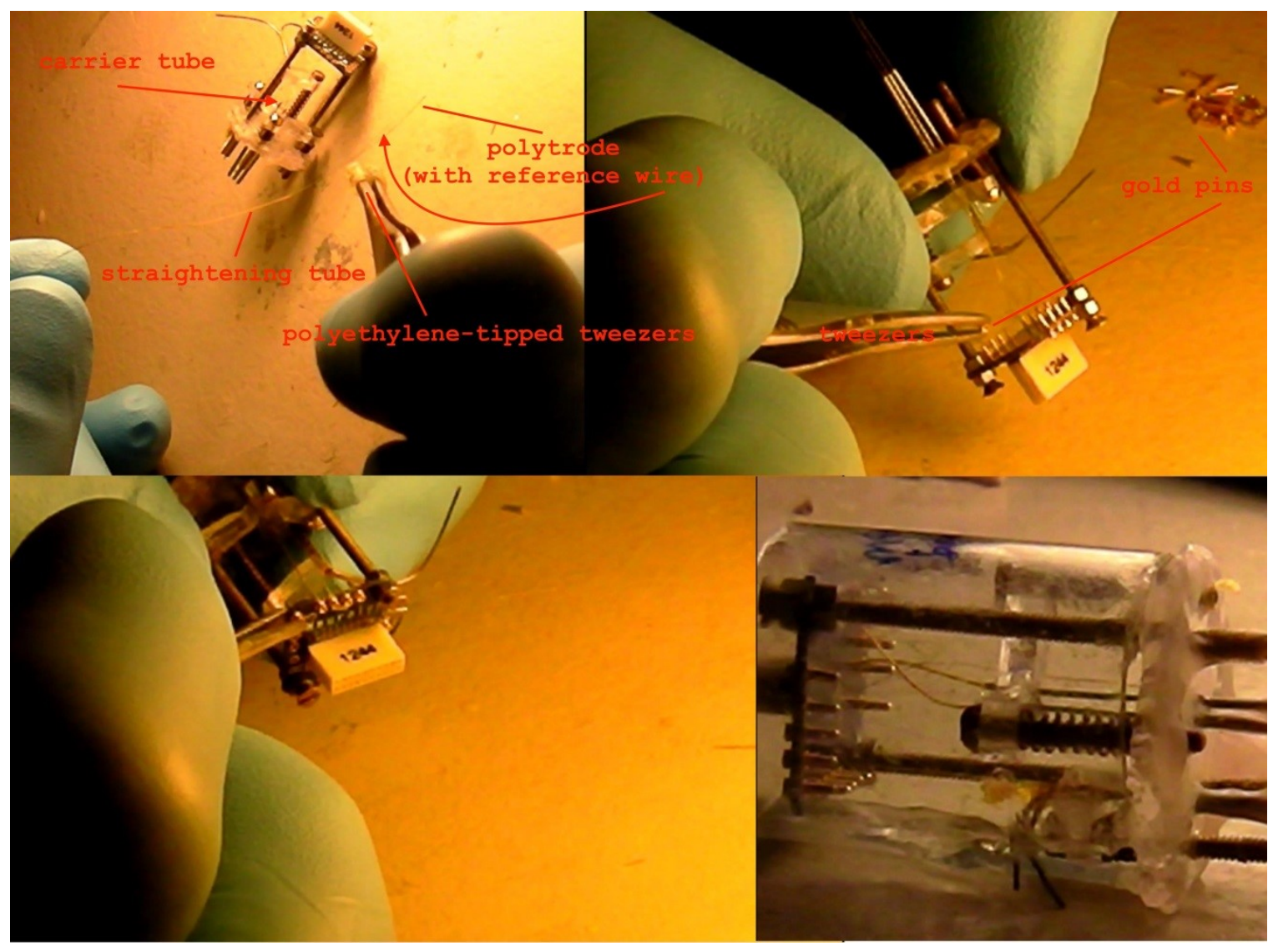

Figure 5. Pinning Electrode Wires to Interface Board.Upper left - capturing polytrode for insertion into carrying tube. Upper right - inserting gold pin into interface board receptacle having wire inserted. Lower left - pinching gold pin to wire and into receptacle. Lower right - completed Top-hat u-array showing all wires and tubes finally situated. 


\section{Gold-plating protocol}

The gold-plating protocol essentially mirrors those in common use, but one extra step is borrowed from Ferguson et al, 2009 (whose protocol is otherwise exemplary): the addition of plating inhibitors in order to ultimately accomplish a "rice-like" texture on the electrode tip that reduces impedance without increasing site-exposure, i.e. increases sensitivity without sacrificing selectivity. Briefly, $1.125 \mathrm{~mL}$ of $1 \mathrm{~g} / \mathrm{L}$ PEG-8000 and 0.375 $\mathrm{mL}$ non-cyanide gold-plating solution (gold-plating solution, Neuralynx, Bozeman, MT) are combined in a $1.5 \mathrm{~mL}$ centrifuge tube. In our case, a complementary connector having individual pre-connected output wires accessible for running current through each electrode individually is affixed to the interface board's connector, the polytrodes are all lowered into the solution. The electrode tips are first "cleaned" (metal oxides reduced and some debris repelled) by passing the opposite current as used for plating, i.e. electrode being positive, using $0.1 \mathrm{uA}$ (controlled by a stimulus isolator: in our case, WPI 365) for $<5$ seconds. Next, the polarity is reversed, and plating proceeds through all electrodes in succession using the same current $(0.1 \mathrm{uA})$ for up to 30 seconds during the first round and for progressively shorter durations thereafter until electrode impedance measured $\sim 50-100 \mathrm{kOhm}$; this often required longer duration pulses during successive rounds than those given in the reference. (Figure 6)

Spritzing with alcohol will not ruin the tip and was used as an antiseptic just before surgery (checking the need for re-plating beforehand if waiting $>1$ week for surgery).

\section{During surgery}

In order to ensure accurate placement into final coordinates, registering the array to the stereotaxic apparatus is essential. Without having to assume the entire array has been made with right angles in mind at every fitting and gluing step, it was useful in our case to simply use the connector as an anchor point to the stereotax's holder arm and make slight adjustments there that optimized the colinearity of the guide tubes with respect to the arm (which assumes the guide tubes have been aligned with respect to each other), e.g. by holding the arm against a straightedge and comparing the guide tubes to the same edge. The anchor point consists of a customized screw-in extension to the holder arm that clamps onto the connector via a screw-on faceplate; although the screws must be tightened as little as possible, the force holding the Top-hat u-array should not be overcome by gentle tugs on the ground wire. (Figure 1)

After preparing the animal for implantation following standard rodent surgical procedures -specifically after accurate formation of trephine holes for all necessary components ( 2 or 3 skull screws + up to 4 polytrode holes)-, the pre-registered array/arm combination is affixed to the stereotax, carefully lowered until guide tubes are $\sim 1 \mathrm{~cm}$ above the skull, and sterile petrolatum or antibiotic ointment applied to each polytrode base at the guide tube (in order to prevent the entry of dental cement later). The main 


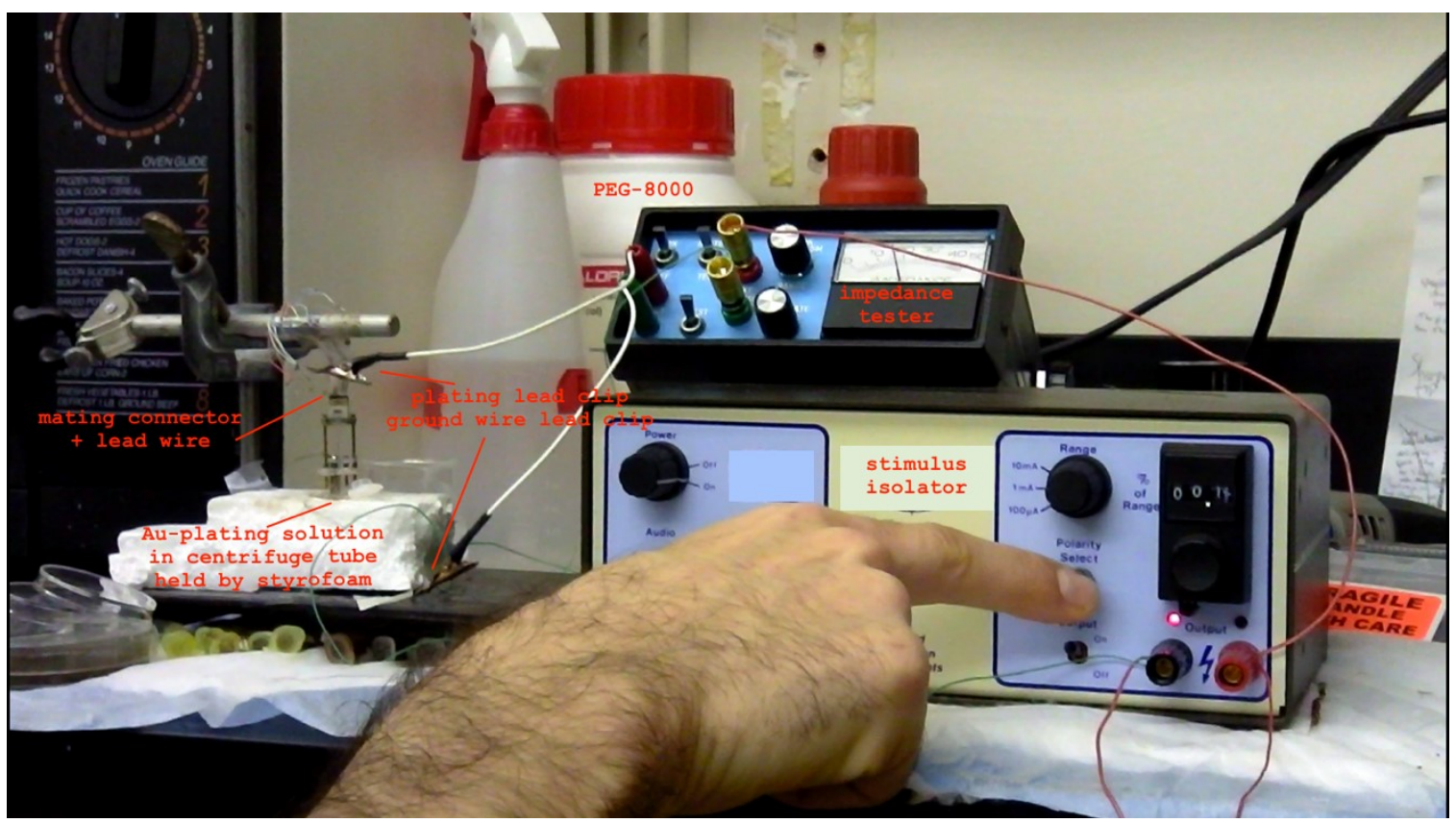

Figure 6. Gold-Plating Electrodes.

Styrofoam holding plating solution in centrifuge tube, impedance tester connected to wire in solution (green lead) and individual electrode (colored mating connector wires wrapped around stand arm), changing polarity on right to plating (from cleaning) 
ground wire is then wrapped around a skull screw -as far from the polytrodes as possible in order to facilitate later work with them-allowing some slack in order to enable slight retractions in case polytrodes need to be slightly repositioned.

In order to facilitate polytrode entry into the brain, it may be necessary to cut dura; in our case, the smallest syringe tip available having been slightly curved at the tip was used to pierce and retract dura, which requires careful monitoring of bleeding (and use of epinephrine and/or gelfoam and saline flushing / sterile swabbing as necessary). Taking note of the position on the stereotax ruler as each polytrode reaches the dura during penetration allows one to reconstruct (using the final position measurement) the depth that each polytrode has been placed below dura. Dental cement can then be draped from each guide tube at its protrusion from the base of the Top-hat u-array to its situation on the animal's skull, and liberally applied to all skull screws and any available anchoring screw tips; ensuring that all locations having cement eventually connect via cement to all others reinforces the integrity of the entire interface. Allow the dental cement to harden to the touch before unscrewing the faceplate that holds the connector as gingerly as possible and returning the animal to a recovery cage. No special precautions are necessary for the animal's wellbeing in the new cage except that it should be housed alone.

\section{Recovering Top-hat u-array parts}

Figure 7 pictures the Top-hat u-array after removal from a sacrificed animal. It is advisable to recover parts that are hard to find and/or expensive (in our case, the EIB-16 and 1-inch screws). Pliers can easily remove most large chunks of dental cement that has not been soaked in methyacrylate for softening. Smaller chunk in the grooves of screws (and large chunks that threaten the integrity of fragile parts) can be softened or carefully removed using finer tools.

It is also advisable to inspect the polytrode tips and protrusions (e.g. to inform depth estimates taken during recording), then unscrewing the microdrives - upward- to minimum protrusion, taking a measurement, and comparing to original parameters in order to check for evidence of drive, glue or tube failure before screwing downward to maximum protrusion for same.

\section{Pitx3Null Mouse Model}

Pitx3Null mice lack a functioning Pitx3 transcription factor, which ultimately leads to the death of the vast majority of SNc tyrosine hydroxylase positive (i.e. DA producing) neurons and DA receptor supersensitivity in the striatum (Hwang et al, 2005), while some VTA DA neurons are independent of Pitx3 and thus survive (Jacobs et al, 2007; Hwang, 2003). This loss of DA neurons that would otherwise project to the dorsal striatum yields a dorsoventral DA denervation gradient in the striatum having the following 


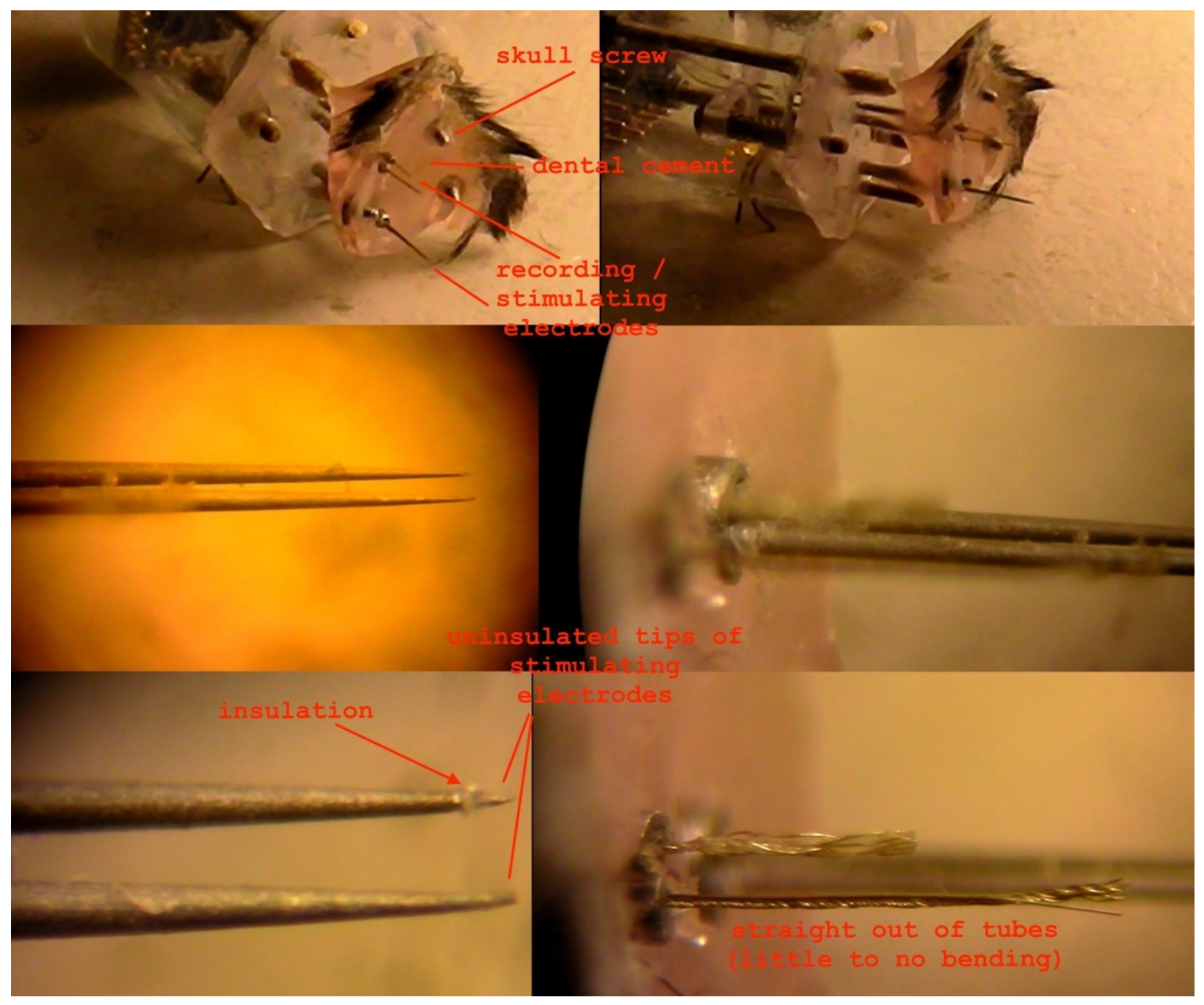

\section{Figure 7. Recovered Top-Hat U-Array Used for Parameter Checks after Months of Implantation.}

Upper left and right - differing angles of whole Top-hat u-array pulled from skull after sacrifice Middle left - 10x magnification of stimulating electrode set tips Middle right 40x magnification of stimulating electrode set protruding from guide tube Lower left40x magnification of stimulating electrode set tips Lower right - 40x magnification of polytrode tips protruding from base (splay only slightly larger than when implanted), reference wire accompanies lower polytrode deinsulated to $1 \mathrm{~mm}$ from tip. 
characteristics: relative to WT siblings, the nucleus accumbens (ventral striatum) retains $\sim 40-80 \%$ of its releasable DA from $\sim 30 \%$ of the midbrain DA-ergic axons; however, the dorsal striatum retains $\sim 0-10 \%$ of its releasable DA from DA-ergic terminals totalling $<10 \%$ of WT terminals (Smits et al 2007, Li \& Zhou, 2013, Wei et al. 2013). This denervation gradient is associated with molecular markers of DA receptor supersensitivity in the dorsal striatum subsequent to 1-DOPA in common with similar animal models (Francardo \& Cenci, 2014).

\section{Recording Animal Motor Behavior before and after DA Agonism}

An animal protocol guiding this work was approved by the University of Tennessee Health Science Center's Laboratory Animal Care Unit's Institutional Animal Care and Use Committee. Male Pitx3Null and WT mice aged $6(+/-4)$ months were used for all experiments as follows. A mouse was recorded beginning $\sim 1$ week after microdrive implantation while tethered to a commutator via lightweight headstage cable (Omniplex, Plexon Inc., Dallas, TX). A baseline saline injection was given 10 min after recording began, and the animal was allowed to explore the $\sim 8$ " $x 8$ "x 8 " cage (having no top, see Figure 8). After $\sim 80 \mathrm{~min}$. had passed from recording start, 1-DOPA (25 mg/kg with $5 \mathrm{mg} / \mathrm{kg}$ benserazide) or D1 agonist (1.0 mg/kg SKF 81297, higher doses tended to lead to epilepsy in Pitx3Null animals; this dose also minimized WT responses without going below our known significant responses in Pitx3Null mice from Li \& Zhou, 2013) was injected, and 160 (1-DOPA) or 80 (D1 agonist) min were allowed to pass before the recording ended or another drug was delivered (for qualitative comparison). Doses for quantitative comparisons were given $\sim 24 \mathrm{~h}$ apart from each other.

\section{Electrophysiological Recordings}

On each animal a microdrive array containing $16 \mathrm{Au}$-plated-nichrome electrode channels in various combinations of poly-trodal configuration (bundles consisting of between 4 and 8 wires) was implanted. A stimulating electrode was included in some microdrives for stimulating antidromic spikes from the substantia nigra reticulata in order to identify striatonigral SPNs. Surgery was performed under ketamine-xylazine ( 90 $\mathrm{mg} . / \mathrm{kg}$. ketamine $+10 \mathrm{mg} . / \mathrm{kg}$. xylazine in $1 \mathrm{uL} / \mathrm{g}$. .9\% saline) anesthesia following standard procedures for rodent survival surgery (e.g. Richter et al, 2013).

Raw recordings were band-pass filtered in the acquisition software (Omniplex Server, Plexon Inc., Dallas, TX) 100 - 8000 Hz. Units were detected and classified by their thresheld energy. Initially, putatively single unit clusters in various 3-dimensional metric spaces (e.g. using principal components, non-linear energy, time slices, etc. in Offline Sorter v3, Plexon Inc. Dallas, TX) were used to make unit templates, which were used as the basis for template-based sorting of remaining waveforms. Previously

published criteria utilizing auto-correlations (not shown), inter-spike interval histograms, and spike waveshape were used for classifying units as SPN, or non-SPN (e.g. Mallet et 


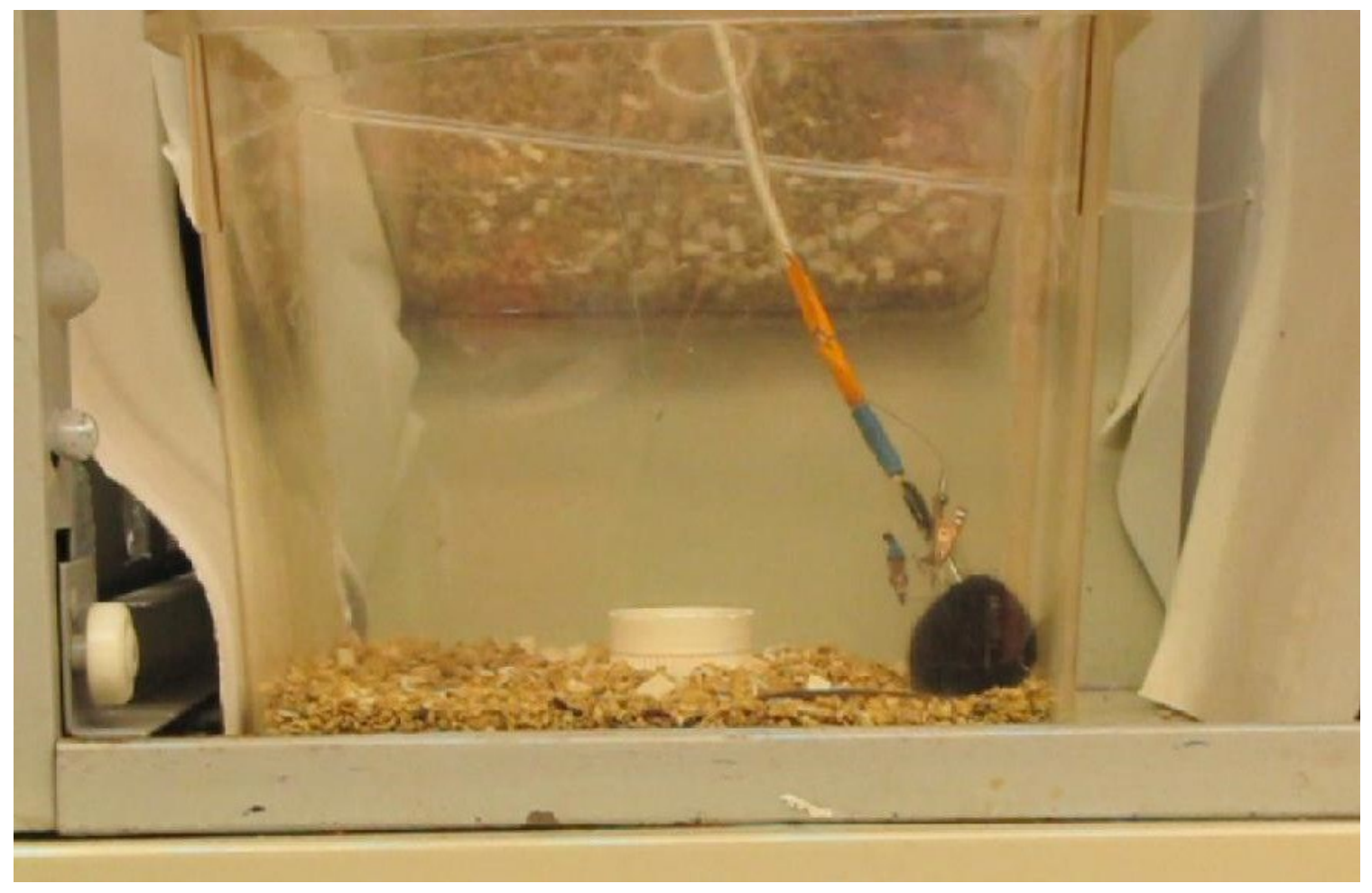

Figure 8. The Open Field Cage.Placed in front of a mirror inside a hollowed out filing cabinet, an 8 " $\mathrm{x} 8$ " $\mathrm{x} 8$ " clear plastic cage having no top served as the mouse's open field. A cap of water is in the middle of the cage at the moment, although this was not routine. See text for details. 
al, 2005, Schmitzer-Torbert and Redish, 2008). (Figure 9) All publication quality SPNs were characterized by ellipsoids of 1.96 standard deviations (as set in Offline Sorter) being out of contact with the nearest unit ellipsoids in a choice feature space (usually principle component space), having $<0.1 \%$ of spikes violating a refractory period of 2.0 msec, and $>90 \%$ of spikes recovered (inferred by comparing the distribution of valley values to an expected gaussian distribution; data not shown). An exemplary recording, units, and ID metrics are shown in Figures 10-13.

\section{Antidromic Identification of Single Units}

In order to ascertain the role of D1-SPNs here, they were putatively electrophysiologically identified in the hopes of finding them among those increasing activity (consistent with the canonical rate model) and to look for any evidence that their activity opposed expectations. Striatonigral (D1 receptor-expressing) SPN identification was accomplished using antidromic spikes elicited by stimulating the substantia nigra (Ryan et al, 1989); because this technique likely produces false negatives (Mallet et al, 2006) and because irresolvable cells responding to stimulation may confound interpretation, commentary concerning the subset of identified cells is restricted to those that responded unambiguously (better isolated than most and with minimal overlap from co-responding units) to nigral stimulation and is not necessarily representative of the entire population of striatonigral SPNs. Serendipitously, we also found evidence of antidromic spikes in fibers of passage (detailed in the second portion of this study).

A parallel bipolar electrode having uninsulated tips $0.10 \mathrm{~mm}$ long and spaced 0.25 mm (WEST30.1A10; Microprobes, Gaithersburg, MD) was included in some microdrives for implantation into the $\mathrm{SNr}$, at $3.25 \mathrm{~mm}$. posterior to bregma, 1.28 and 1.53 $\mathrm{mm}$ lateral to midline, and $4.25 \mathrm{~mm}$ below dura. Stimulation of all nearby excitable neuronal elements using current intensities varying between 100-500 uA elicited antidromic spikes. Stimulation patterns consisted of either x number of biphasic pulses $0.5 \mathrm{msec}$ in total duration having varying interstimulus intervals as noted, or of $\mathrm{x}$ number of monophasic pulses of $0.05 \mathrm{msec}$ total duration having varying interstimulus intervals, as noted; trains were applied every 20 seconds using a stimulus isolator (A-365; WPI, Sarasota, FL) triggered by a Master 8 (A.M.P.I., Jerusalem, Israel). These responses were recorded in the striatum and identified as either potential direct pathway SPNs (based on SPN metrics and saved for the first portion of the study) or possible fibers of passage (and saved for the latter portion of the study) if the antidromic response fit the following criteria: lower response latency (putative fibers $<2 \mathrm{msec}$., SPNs $\sim 6-13 \mathrm{msec}$.) time-locked to the stimulation, and an all-or-none quality at peri-threshold stimulation currents. For all SPNs and three of the four fiber-like units of interest reported responding, further evidence was collected: an absolute refractory period $<2 \mathrm{msec}$, and in collisions with spontaneous orthodromic spikes. A schematic and exemplary responses to stimuli are 

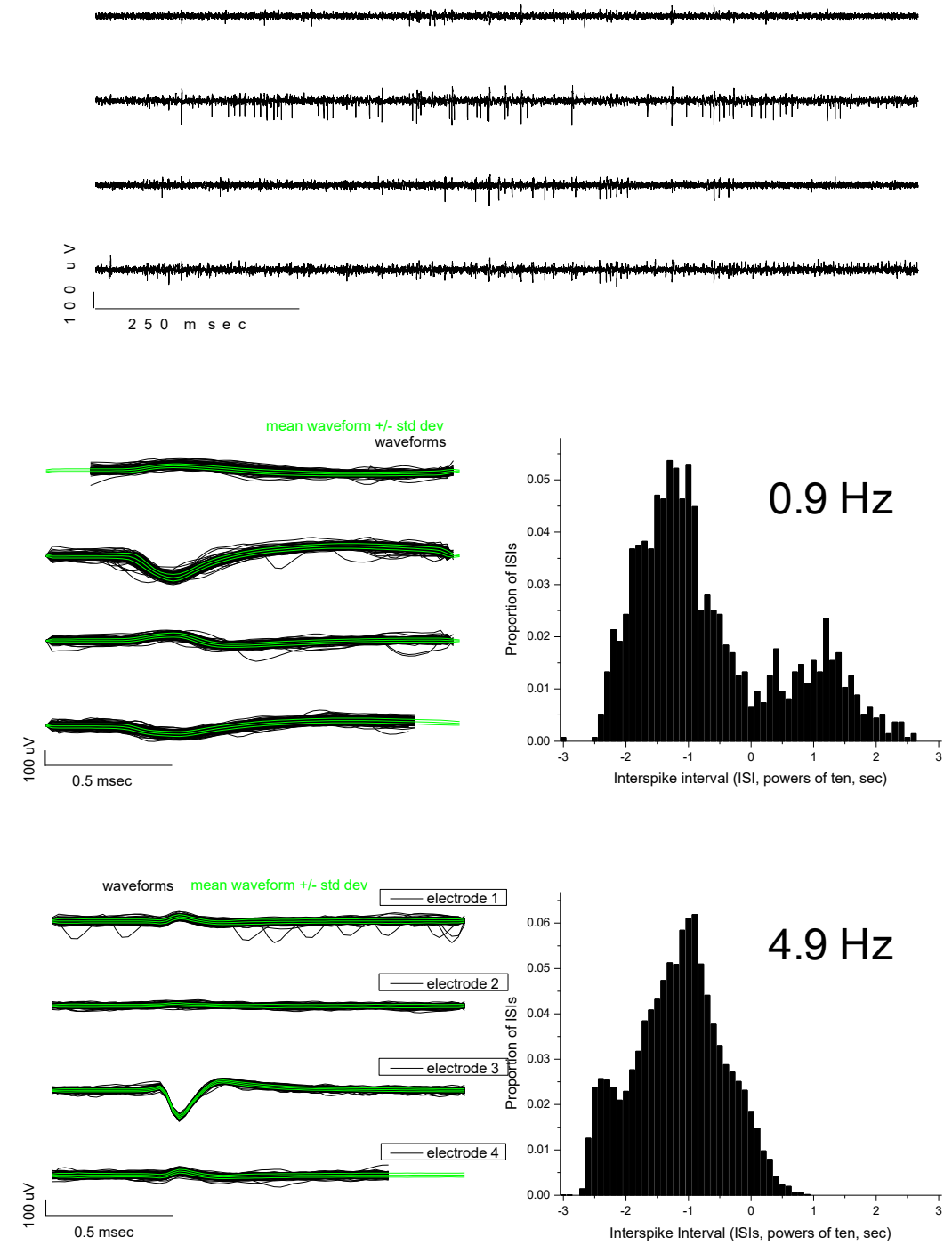

Figure 9. Raw Data and Isolated Units.Upper panel - raw data (vertical sub-panels represent electrodes 1-4 from top to bottom)Lower left panels - waveforms from a putative fiber (middle) and SPN (bottom) that responded to stimulation with antidromic spikes. Lower right panels - ISI histograms from respective units to the left.Narrow waveform (full-width at half-max $<0.2 \mathrm{msec}$ ) and many fewer pause durations $>1 \mathrm{sec}$ would normally preclude this neuron from being considered projection type, (i.e. SPN, the only units that project outside the striatum), and most would consider its mean rate $(\sim 5 \mathrm{~Hz})$ to be fast enough firing to qualify it as a FSI. The lower unit is putatively identified as SPN due to the waveshape ( $>0.2 \mathrm{msec}$, much longer than putative FFNs) and the log-ISI histogram having an approximately bimodal ISI distribution with several pauses longer than one second and as long as hundreds of seconds (as distinct from TANs, which otherwise share waveform length and but have a reliably leptokurtic unimodal log ISI distribution). 

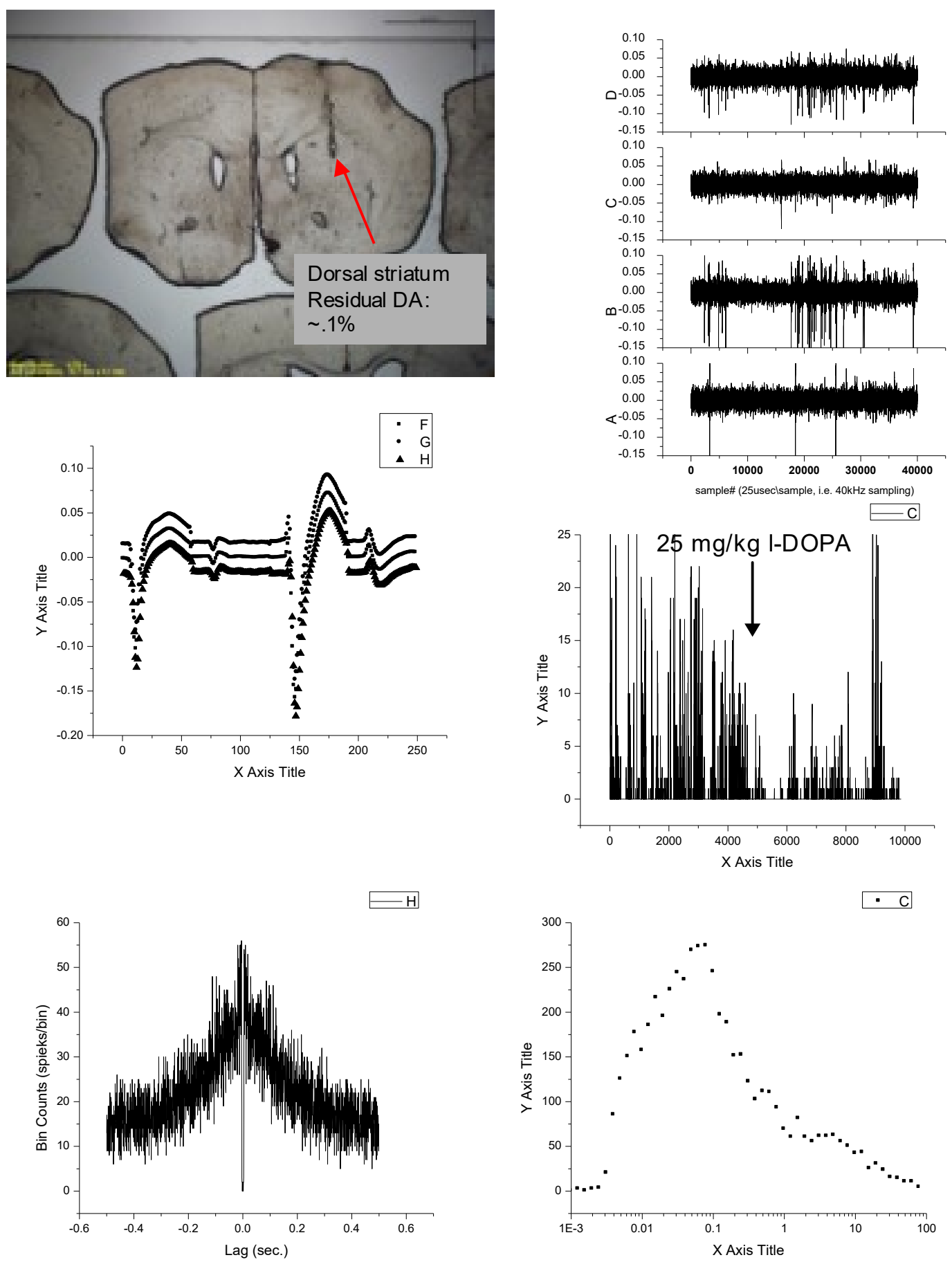

Figure 10. Exemplary Dorsal WT Data under l-DOPA.

Upper right: lesion in non-/dorsal striatum. Upper left: raw data from a recording in this locale. Middle right: average (+/- std) of one isolated unit's wavefrom from the recording.. Middle left: spike rate of this unit over experiment time (1 sec. bins). Lower right: autocorrelogram of this unit's spike activity ( $5 \mathrm{msec}$ bins). Lower left: interspike interval (ISI) histogram of this unit's spike activity (log-plot, 10 non-linear bins per decade) 

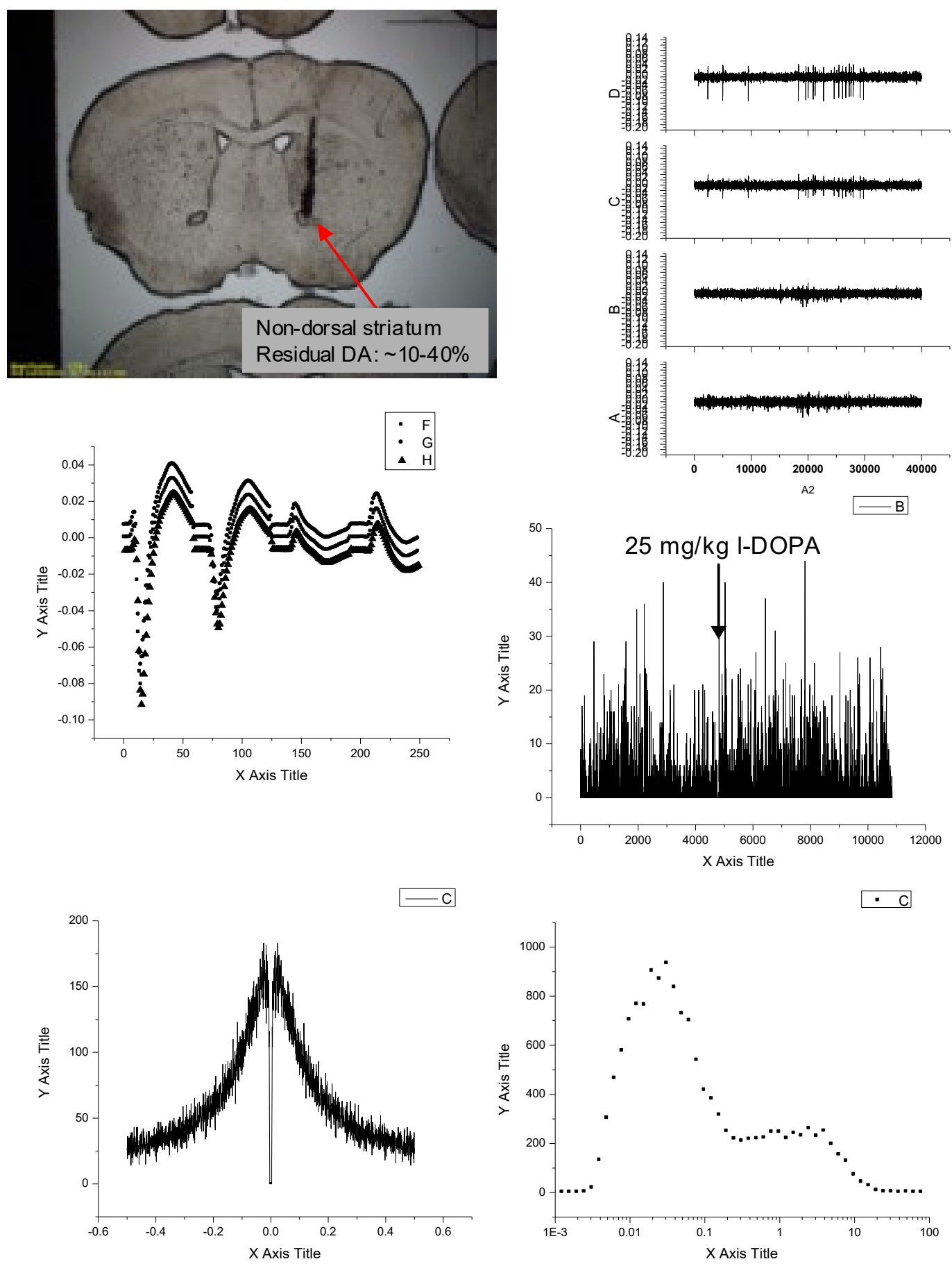

Figure 11. Exemplary Non-Dorsal WT Data under l-DOPA.

Upper right: lesion in non-/dorsal striatum. Upper left: raw data from a recording in this locale. Middle right: average ( $+/$ std) of one isolated unit's wavefrom from the recording. Middle left: spike rate of this unit over experiment time (1 sec. bins). Lower right: autocorrelogram of this unit's spike activity ( $5 \mathrm{msec}$ bins). Lower left: interspike interval (ISI) histogram of this unit's spike activity (log-plot, 10 non-linear bins per decade) 

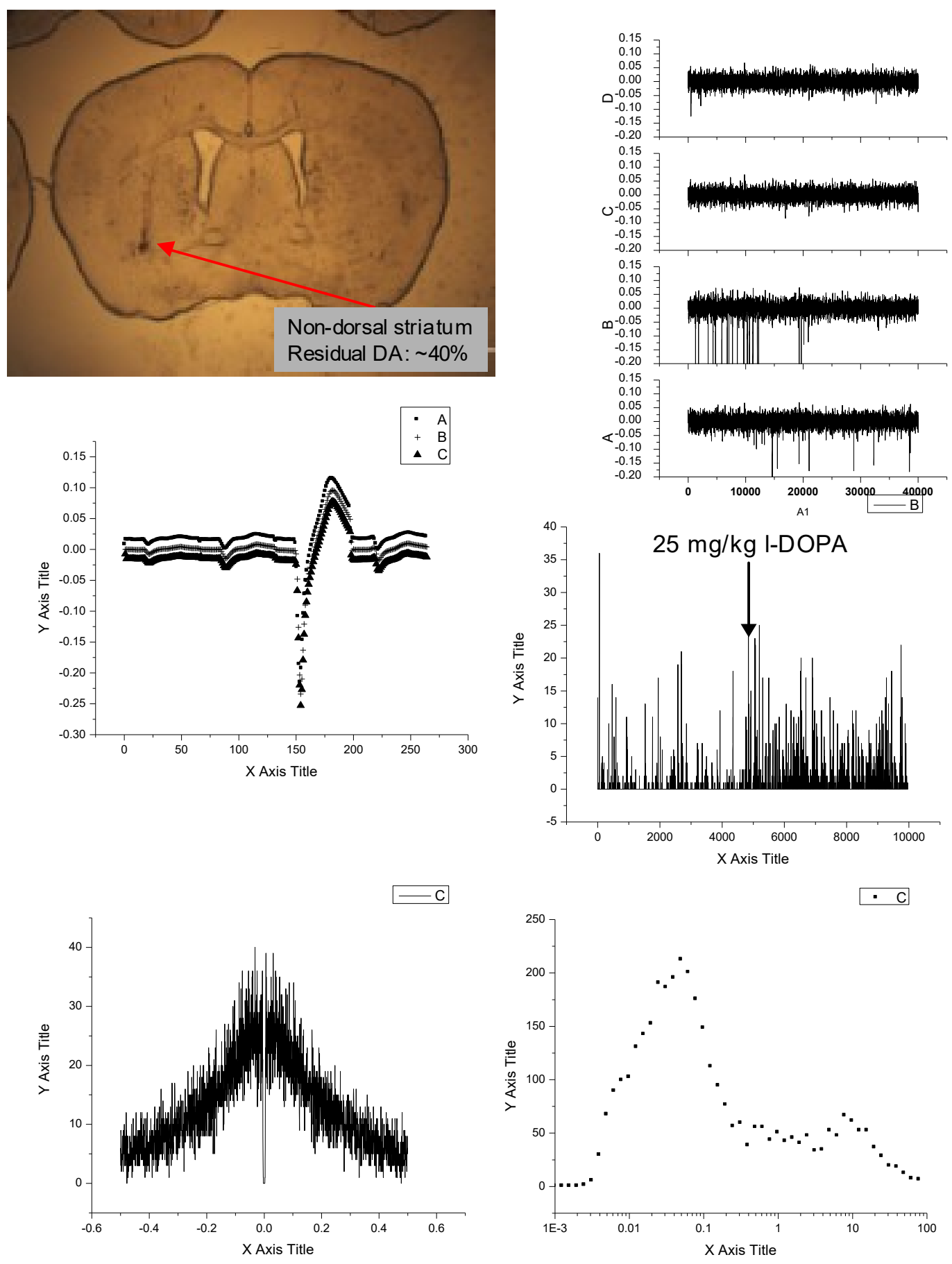

Figure 12. Exemplary Non-Dorsal Pitx3Null Data under l-DOPA.

Upper right: lesion in non-/dorsal striatum. Upper left: raw data from a recording in this locale. Middle right: average (+/- std) of one isolated unit's wavefrom from the recording . Middle left: spike rate of this unit over experiment time (1 sec. bins). Lower right: autocorrelogram of this unit's spike activity ( $5 \mathrm{msec}$ bins). Lower left: interspike interval (ISI) histogram of this unit's spike activity (log-plot, 10 non-linear bins per decade) 

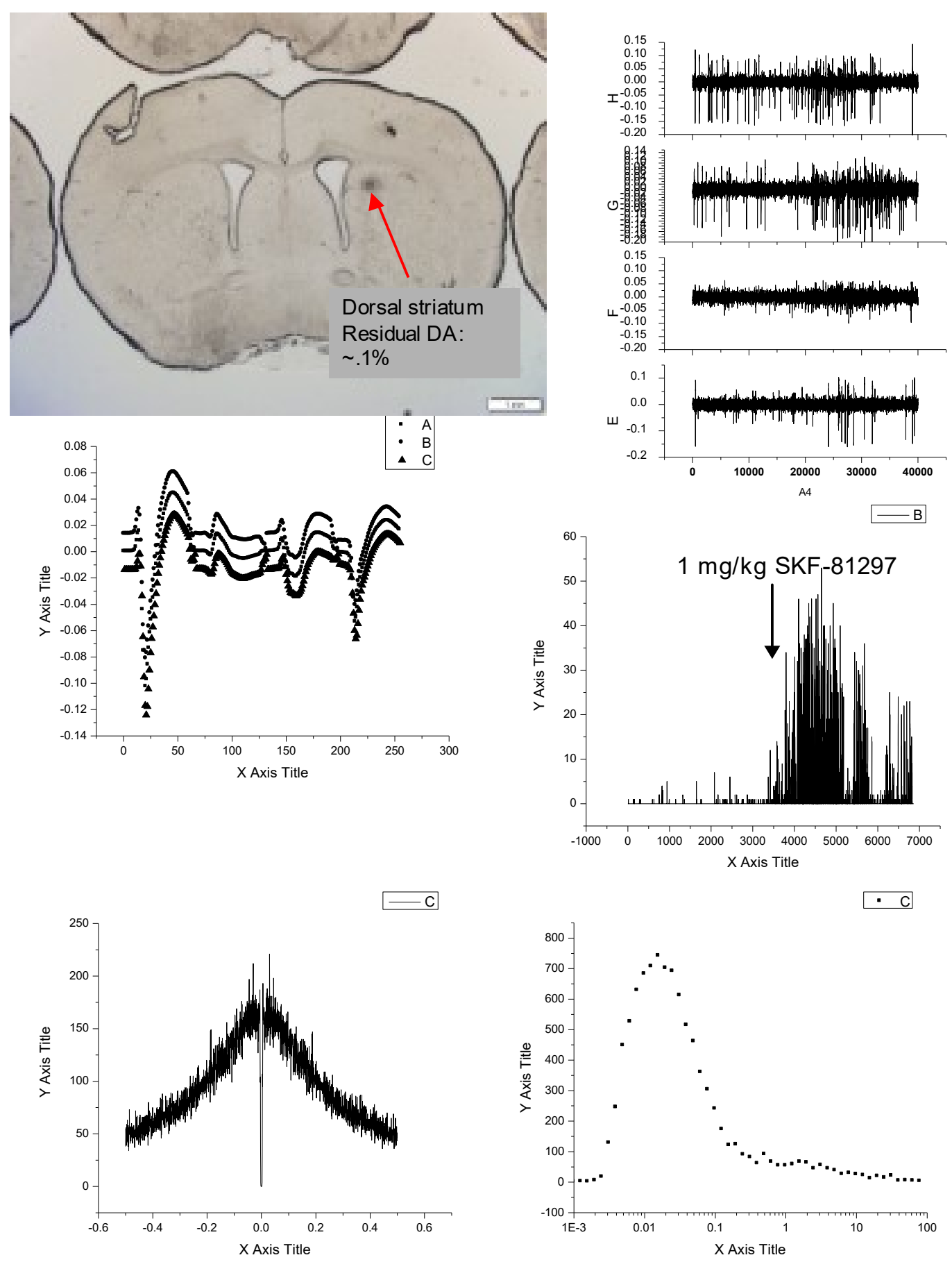

Figure 13. Exemplary Dorsal Pitx3Null Data under D1 Agonist.

Upper right: lesion in non-/dorsal striatum. Upper left: raw data from a recording in this locale. Middle right: average (+/- std) of one isolated unit's wavefrom from the recording . Middle left: spike rate of this unit over experiment time (1 sec. bins). Lower right: autocorrelogram of this unit's spike activity ( $5 \mathrm{msec}$ bins). Lower left: interspike interval (ISI) histogram of this unit's spike activity (log-plot, 10 non-linear bins per decade) 
shown in Figure 14 and throughout the results. Reduction in the variability of response latency shortly following (less than hundreds of msec) any spikes (previous antidromic spikes in this case) may also be seen in some cases (Swadlow et al, 1978; Ryan et al ,1989). SPNs were considered unidentified if any criterion was not met; exemplary collisions are shown in Figure 15. Corecorded fiber-like units having similar characteristics but not responding to stimuli are also presented as unidentified non-SPN units.

\section{A Note on Technical Differences Between the Two Studies}

For the latter study on putative fibers of passage two mice were used, both male and aged 4-6 months: one C57bl/6 and one Pitx3Null. On each animal a microdrive array was implanted aiming octrodes at the dorsolateral striatum (AP +.4, ML 2.0 - 2.5, DV 1.6 $-2.0 \mathrm{~mm}$. below dura). All other parameters were the same as the first study minus the dosing of drugs and subsequent comparisons.

\section{Statistical Considerations}

Each SPN's baseline was calculated as the mean number of spikes per second by counting all spikes recorded during the pre-drug period (defined from the saline injection +35 seconds through the pre-drug-injection period until 35 seconds before drug injection) and dividing by the duration of said period. Each SPN's response polarity was calculated as the sign of the response intensity before taking it's absolute value (thus, a negative indicated a decrease in mean spiking and a positive indicated increase.) Each SPN's response intensity to a drug injection was calculated as the absolute value of the difference of the base 10 logarithm of pre-injection (i.e. baseline) from post-injection (i.e., response) mean rates, i.e. $\log _{10}$ (post mean rate) $-\log _{10}$ (premean rate) $=\log _{10}$ (post mean rate / premean rate). The threshold depth for considering a unit as dorsal or non-dorsal was $2.25 \mathrm{~mm}$ below dura.

Various metrics including mean baseline rate, response polarity (i.e., increasing or decreasing mean rate), and $\log$ (post/pre mean rate) from every recorded SPN were calculated in and exported from Matlab (The Mathworks, Inc, Natick, MA) to an *.xls file. SAS software, version 9.4 (SAS Institute Inc., Cary, NC, USA ), running (as with all other software) under Windows 7 Enterprise, was used to import this *.xls file and to perform subsequent statistical modeling.

Given a potential (and later verified -data not shown) correlation among SPN metrics within animal, a mixed modeling approach was taken using the GLIMMIX procedure in SAS. Thus, mean log-transformed baseline rates (taking each animal's largest sample of neurons between one or the other dataset, 1-DOPA or D1 agonist), response polarity (per dataset) and $\log _{10}$ mean rate change (per dataset) were modeled separately from each other. Model building started with the full 3-way interaction (i.e., 

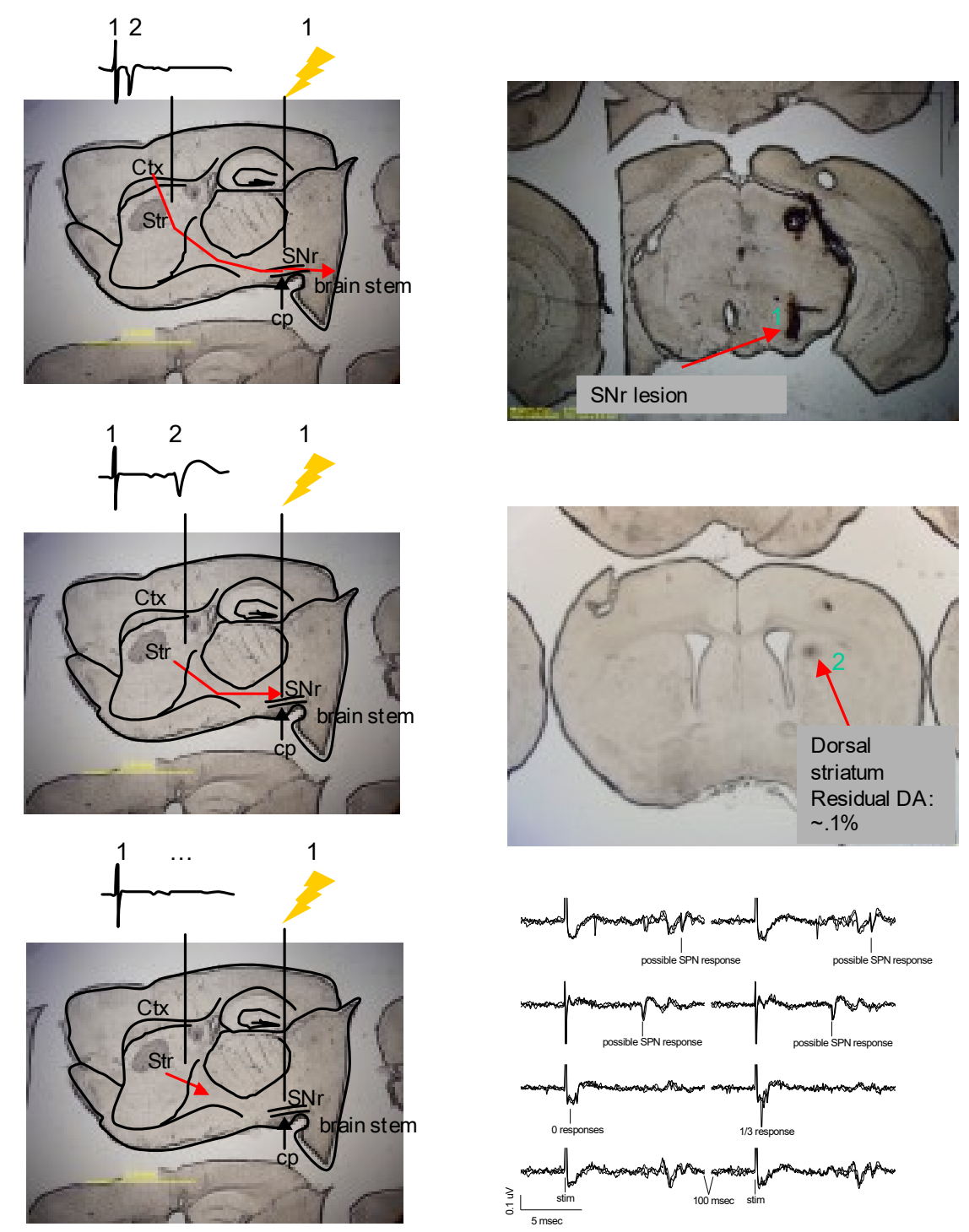

Figure 14. Antidromic Spike Technical Theory.

Left panels from top to bottom - Mouse parasagittal brain sections and overlaid traces of major structures show that because cortical axons pass through the cerebral peduncle (cp) and terminate after the $\mathrm{SNr}$, their axons may transmit an antidromic spike (2) past a striatal recording electrode (1) to the cortical soma during $\mathrm{SNr}$ (and thus potentially cp) stimulation; this response would be expected to happen faster than in SPNs due to saltatory conduction and larger axon diameter, but pass to the $\mathrm{SNr}$ directly. Striatopallidal neurons would not be expected to respond to stimulation at all due to their field of projection stopping short of the SNr. Upper and Middle right panels - Coronal mouse brain sections highlight representative $\mathrm{SNr}$ stimulation (1) and striatal recording (2) sites. Lower right panel - Representative short-latency $(-3.5-+11.5 \mathrm{msec})$ responses to nigral stimulation (note representative all-or-none quality of earliest response expected from antidromic spikes at peri-threshold stimulation current). 

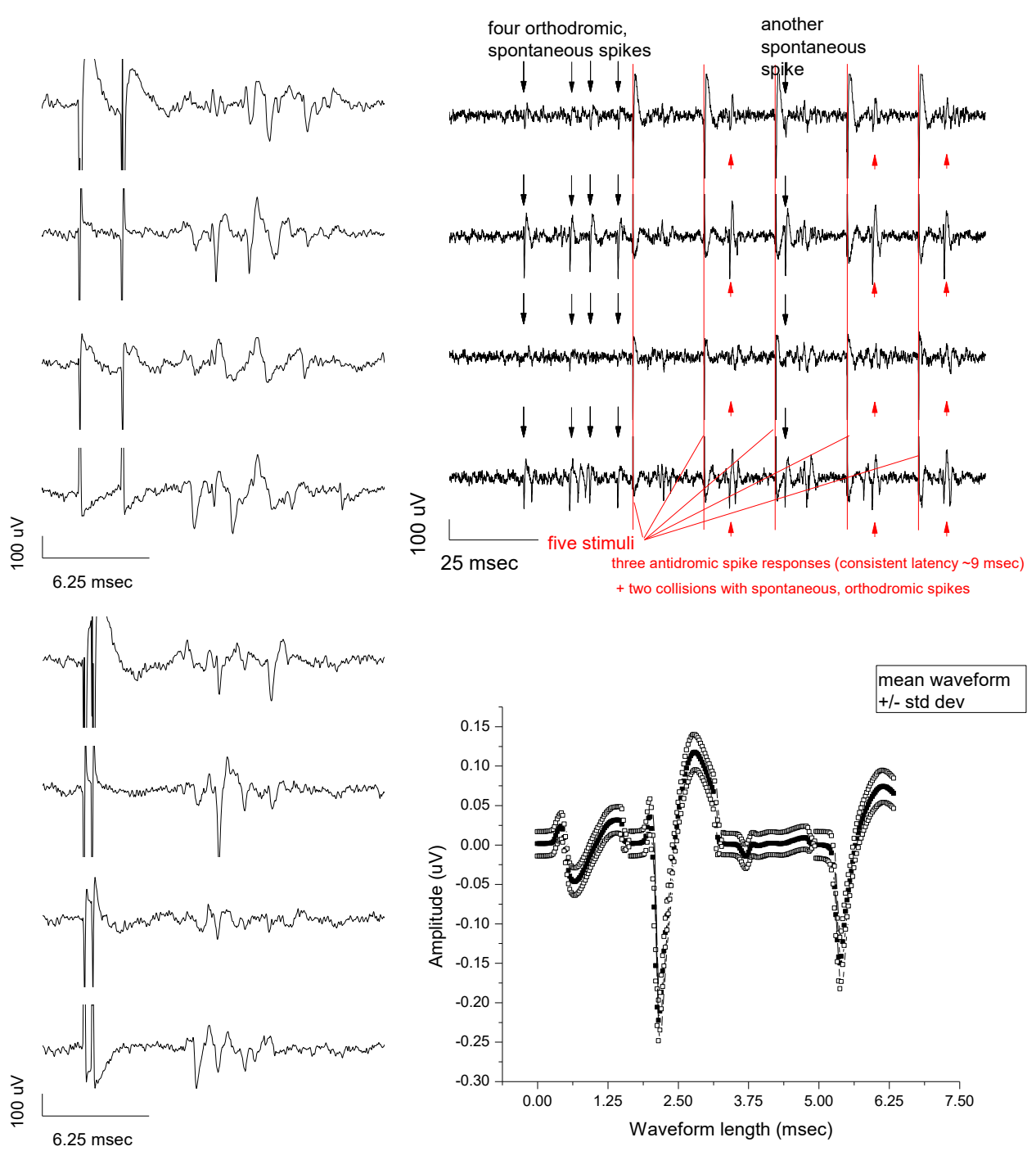

Figure 15. Representative Antidromic Identification Criteria and Collisions.

Upper left - Nigral stimuli $2.0 \mathrm{msec}$. apart elicit two corresponding spike responses at the recorded SPN soma Lower left - Same using $0.5 \mathrm{msec}$ interstimulus interval in order to show single response Upper right - Collisions from the same unit's antidromic spikes with its spontaneous orthodromic spikes are evident in the first first and third stimuli and not in those remaining during which no spontaneous orthodromic spikes occurred (note consistent latency of antidromic responses) Lower right - The mean waveform $+/$ standard deviation of the responding SPN is shown. 
baseline*genotype*depth + all 2-way interactions and main effects), which if insignificant was followed by the model having the 3-way without the insignificant 2way interactions. If the 3 -way interaction was not significant, then all two way interactions were included and modeling proceeded by removing highest order most insignificant interactions before moving to the final modeling phase. Since responses from most animals came from only one depth out of two possible, then there was little overlap within animal over depth coverage, i.e., only 3 of 19 animals yielded responses from both depths. Thus, degrees of freedom for F-tests and post-hoc analyses were calculated using SAS's updated Kenward-Roger's adjustment called "kr2" in the "ddfm" option of the "model" statement. Baselines, 1-DOPA response polarities (as a binary response) and intensities (as a continuous response), and D1 agonist response polarities (as a binary response) and intensities (as a continuous response) were modeled separately using the above approach.

Only three post-hoc comparisons were planned: the effect of a unit change in baseline rate on dorsal Pitx3Null drug responses, the effect of genotype on dorsal responses (i.e. Pitx3Null dorsal v. WT dorsal) and the effect of depth within Pitx3Null animals (i.e. Pitx3Null dorsal v. Pitx3Null non-dorsal responses). Post-hoc comparison pvalues for differences between specific subgroups of the interactions were corrected with a stepdown Sidak adjustment (a.k.a. Holm-Sidak). We report adjusted, unadjusted and Type III p-values as noted below.

In order to mitigate complications involved in reporting the model results, the reader may find it useful to recall the reference and non-reference groups categorized for each factor. "WT" and "non-dorsal" were taken as reference classes; however, for baseline spiking (which is a continuous variable), one $\log _{10}$ unit higher baseline value is taken as the reference value in order to showcase enhancements in the responses that are due to low baselines since correlations with baseline were negative. This allows comparisons of the average non-reference SPN as the group with enhanced response having a baseline one tenth of the average reference (i.e. a $\log _{10}$ unit higher) (or yielding a positive odds ratio -O.R.- by being the numerator). This becomes especially useful when interpreting O.R.s for interactions, for which the factor precedes the ratio; e.g., "baseline O.R. $=1.23^{*}$ " defines an Odds Ratio in which the Odds estimate for the average SPN having a baseline rate one tenth that of the reference group (i.e. nonreference category making up the numerator) is 1.23 times higher than the Odds estimate for the reference group, which is the average SPN having a baseline ten times the nonreference (i.e. reference category making up the denominator). (Odds represent the probability of an increase over that of a decrease and are only explicit in the intercept, i.e., are implicit or factor/subgroup-dependent in O.R.s.) Appending a certain subgroup to the O.R. name (e.g., Pitx3Null depth O.R.) specifies to which subgroup an analysis was restricted. 


\section{CHAPTER 4. RESULTS}

\section{Results for DA Agonism in DA-Deficient SPNs}

Databases are tabulated below (Tables 1 and 2) for the three experiments: baseline comparisons, responses under l-DOPA and responses under SKF 81297.

\section{Basal SPN mean firing in WT and DA-deficient Pitx3Null mice}

In order to ascertain an effect of Pitx3Null genotype on baseline SPN spiking, baseline rates were compared between genotypes and depths. Since the distribution of baseline rates spanned $>3$ orders of magnitude $(0.00097-5.94 \mathrm{~Hz}$; median $0.185 \mathrm{~Hz}$, Table 3 ), $\log _{10}$ (baseline) was used. No significant baseline rate differences between factors were found for this dataset. (Figure 16) Given no spiking distinction for DAdeficient SPNs overall, we explored their responses to DA agonism using 1-DOPA and D1 agonist.

\section{Response polarity differences among WT and DA-deficient Pitx3Null mice}

With respect to the canonical rate model, an overall spiking increase or decrease in response to DA agonists has been used as a metric for subtype identification of SPNs (e.g. Liang et al., 2008, Singh et al., 2016). Having noticed both response polarities after the putatively selective D1-type receptor agonist SKF 81297 (Figure 17), we explored both datasets separately for differences in response polarity ratios, i.e. odds of increases compared to decreases dependent on possibly full interactions of our 3 factors of interest: $\log _{10}$ (mean baseline rate), depth and genotype.

The baselines dataset includes a total of 198 SPNs from 19 animals split into four groups determined by genotype and depth interaction, i.e. WT non-dorsal and dorsal as well as Pitx3Null non-dorsal and dorsal. Seven animals contribute to the dorsal datasets for each genotype (57 SPNs from Pitx3Null animals and 46 from WT), whereas nondorsal data comes from 3 Pitx3Null and 6 WT animals (67 and 27 SPNs, respectively). During experiments, we observed firing rate changes that were not immediately obvious as to whether or not cells increased or decreased activity (Figures 18-21), especially since SPNs can go many minutes without spiking. Thus we assessed the polarity of any change in firing as an increase, -i.e. positive- if the result of subtracting the $\log _{10}$ (mean baseline) from the $\log _{10}$ (mean response) was positive, and negative otherwise. No allowance was made for SPNs having "no change" in activity for two main reasons; this would require testing increases in rate separately from decreases (or removing insignificantly changing SPNs from the dataset, requiring post-hoc classification that nullifies the generality of the model); and there is no particularly well-suited statistical 
Table 1. Datasets Used.

\begin{tabular}{|c|c|c|}
\hline & Pitx3Null SPNs & WT SPNs \\
\hline $\begin{array}{l}\text { Baselines (from subset of non- } \\
\text { overlapping drug recordings) } \\
\text { dorsal } \\
\text { non-dorsal }\end{array}$ & $\begin{array}{l}57 \text { cells in } 7 \text { animals } \\
67 \text { cells in } 3 \text { animals }\end{array}$ & $\begin{array}{l}46 \text { cells in } 7 \text { animals } \\
27 \text { cells in } 6 \text { animals }\end{array}$ \\
\hline $\begin{array}{l}\text { I-DOPA } \\
\text { dorsal } \\
\text { non-dorsal }\end{array}$ & $\begin{array}{l}48 \text { cells in } 7 \text { animals } \\
55 \text { cells in } 3 \text { animals }\end{array}$ & $\begin{array}{l}37 \text { cells in } 6 \text { animals } \\
25 \text { cells in } 4 \text { animals }\end{array}$ \\
\hline $\begin{array}{l}\text { SKF } 81297 \\
\text { dorsal } \\
\text { non-dorsal }\end{array}$ & $\begin{array}{l}32 \text { cells in } 4 \text { animals } \\
59 \text { cells in } 2 \text { animals }\end{array}$ & $\begin{array}{l}33 \text { cells in } 6 \text { animals } \\
29 \text { cells in } 7 \text { animals }\end{array}$ \\
\hline
\end{tabular}

Table 2. Antidromically Identified Cell Subset.

\begin{tabular}{lll}
\hline & \multicolumn{1}{c}{$\begin{array}{c}\text { Pitx3Null SPNs } \\
\text { (antidromically } \\
\text { identified) }\end{array}$} & \multicolumn{1}{c}{$\begin{array}{c}\text { WT SPNs } \\
\text { (antidromically } \\
\text { identified) }\end{array}$} \\
\hline $\begin{array}{l}\text { Baselines (from subset of non- } \\
\text { overlapping drug recordings) } \\
\text { dorsal } \\
\text { non-dorsal }\end{array}$ & $\begin{array}{l}6 \text { cells in } 3 \text { animals } \\
(\mathrm{N} \backslash \mathrm{A})\end{array}$ & $\begin{array}{l}6 \text { cells in } 3 \text { animals } \\
(\mathrm{N} \backslash \mathrm{A})\end{array}$ \\
\hline $\begin{array}{l}\text { I-DOPA } \\
\text { dorsal } \\
\text { non-dorsal }\end{array}$ & $\begin{array}{l}6 \text { cells in } 3 \text { animals } \\
(\mathrm{N} \backslash \mathrm{A})\end{array}$ & $\begin{array}{l}5 \text { cells in } 2 \text { animals } \\
2 \text { cells in } 1 \text { animal }\end{array}$ \\
\hline $\begin{array}{l}\text { SKF 81297 } \\
\text { dorsal } \\
\text { non-dorsal }\end{array}$ & $\begin{array}{l}3 \text { cells in } 1 \text { animals } \\
(\mathrm{N} \backslash \mathrm{A})\end{array}$ & $\begin{array}{l}8 \text { cells in } 3 \text { animals } \\
2 \text { cells in } 1 \text { animal }\end{array}$ \\
\hline
\end{tabular}


Table 3. Baselines between Genotype and Depth.

\begin{tabular}{|c|c|c|c|c|c|c|c|}
\hline Genotype & Depth & N Obs & Minimum & $\begin{array}{c}\text { Lower } \\
\text { Quartile }\end{array}$ & Median & $\begin{array}{c}\text { Upper } \\
\text { Quartile }\end{array}$ & Maximum \\
\hline Pitx & dorsal & 57 & 0.00100 & 0.04337 & 0.43982 & 0.52062 & 4.79883 \\
\hline Pitx & $\begin{array}{l}\text { non- } \\
\text { dorsal }\end{array}$ & 67 & 0.00336 & 0.08496 & 0.16785 & 0.67718 & 5.21594 \\
\hline WT & dorsal & 46 & 0.00097 & 0.07507 & 0.28397 & 0.81032 & 5.94051 \\
\hline WT & $\begin{array}{l}\text { non- } \\
\text { dorsal }\end{array}$ & 27 & 0.01291 & 0.03672 & 0.12216 & 0.28427 & 3.66251 \\
\hline
\end{tabular}

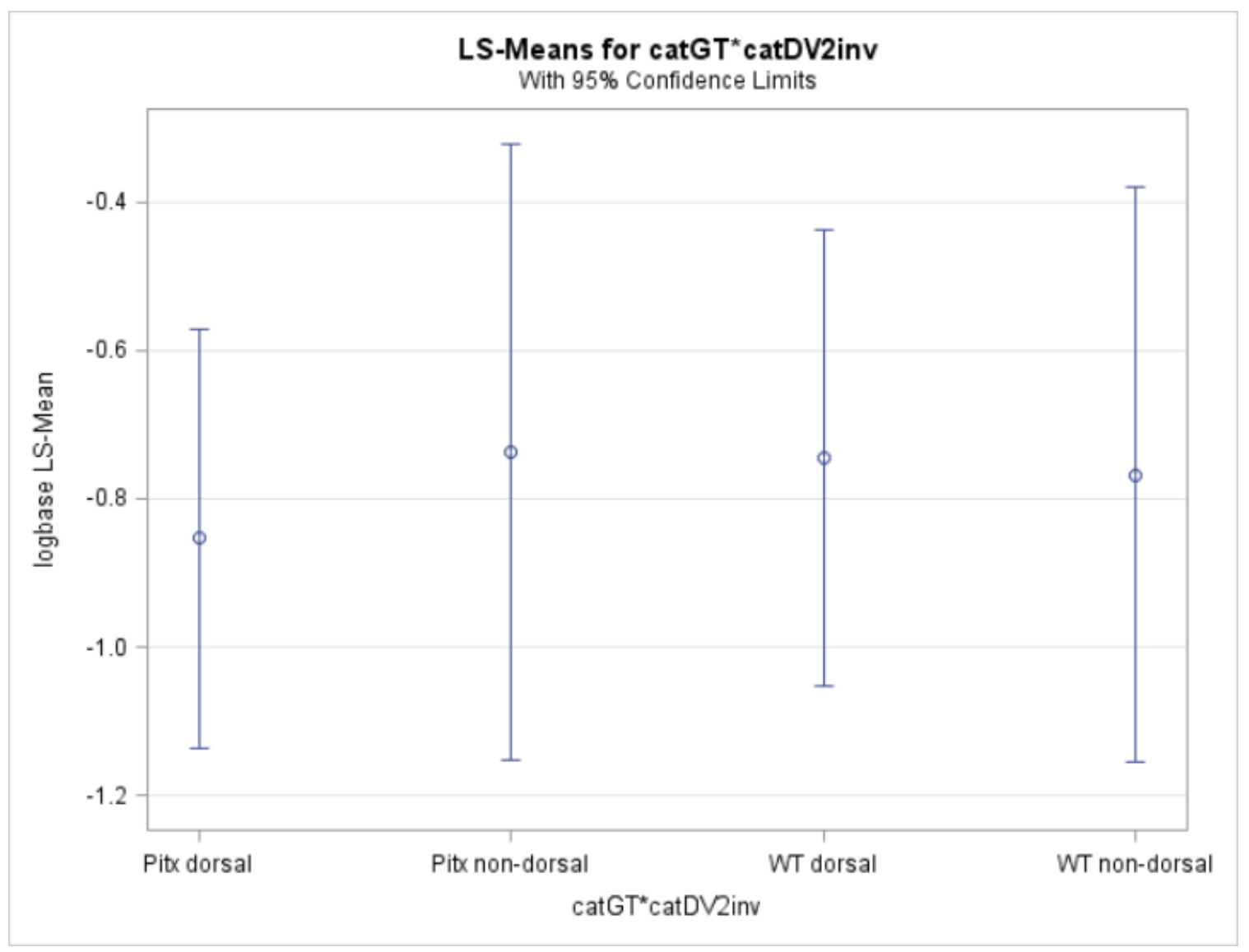

Figure 16. Modelled Mean Baselines +/- Standard Errors for Each Group. Linear y-scale reflects $\log 10$ (baselineModeled means $+\backslash$-respective standard errors for each group. 


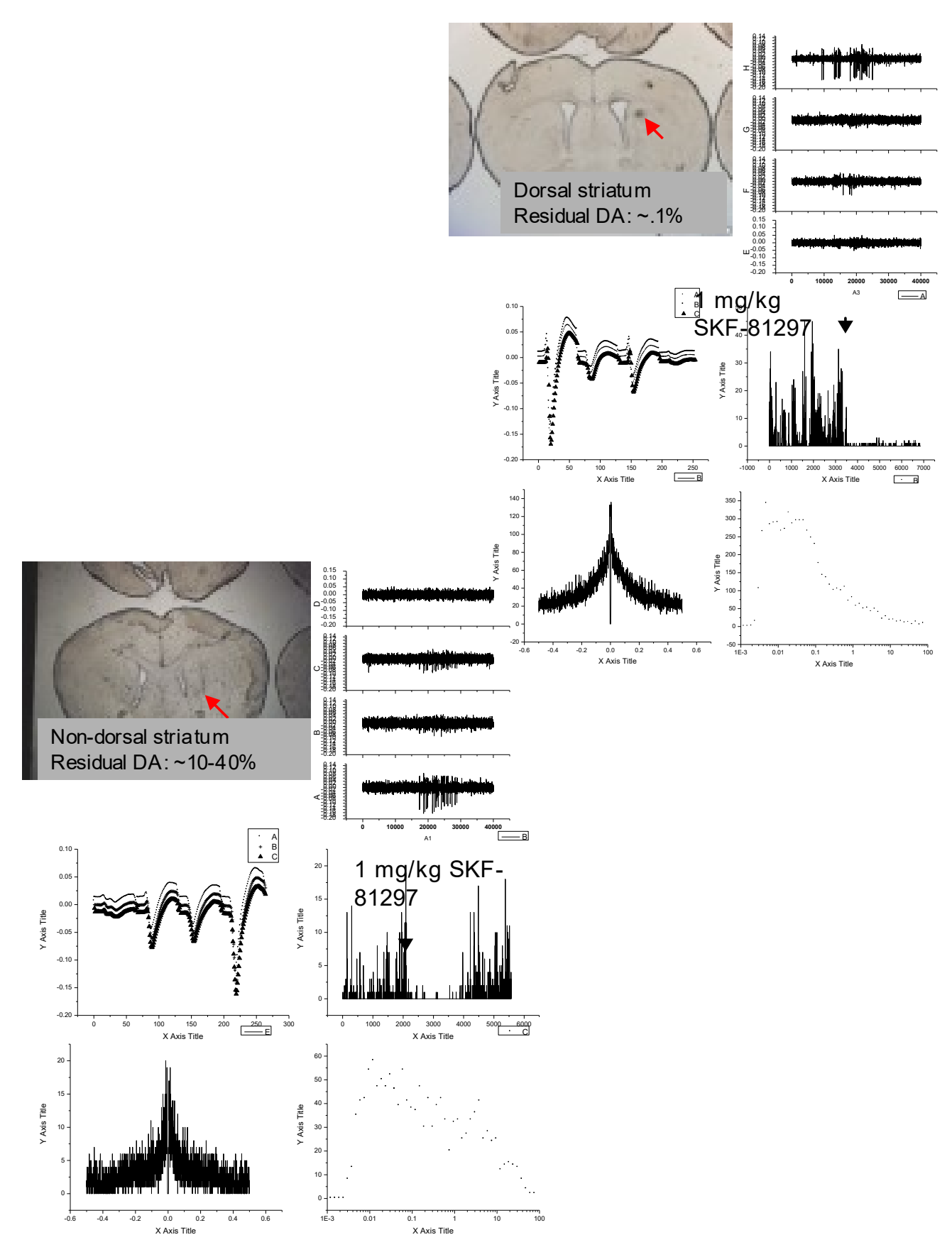

Figure 17. Unexpected Responses to Selective D1 Agonism.

Upper panels - Exemplary dorsal Pitx3Null data under D1 agonism. Upper right subpanel: lesion in dorsal striatum. Upper left sub-panel: raw data from a recording in this locale. Middle right sub-panel: average (+/- std) of one isolated unit's waveform from the recording Middle left sub-panel: spike rate of this unit over experiment time (1 sec. bins). Lower right sub-panel: autocorrelogram of this unit's spike activity ( $5 \mathrm{msec}$ bins). Lower left sub-panel: interspike interval (ISI) histogram of this unit's spike activity (log-plot, 10 bins per decade) Lower panels - same as above but for WT and non-dorsal. 


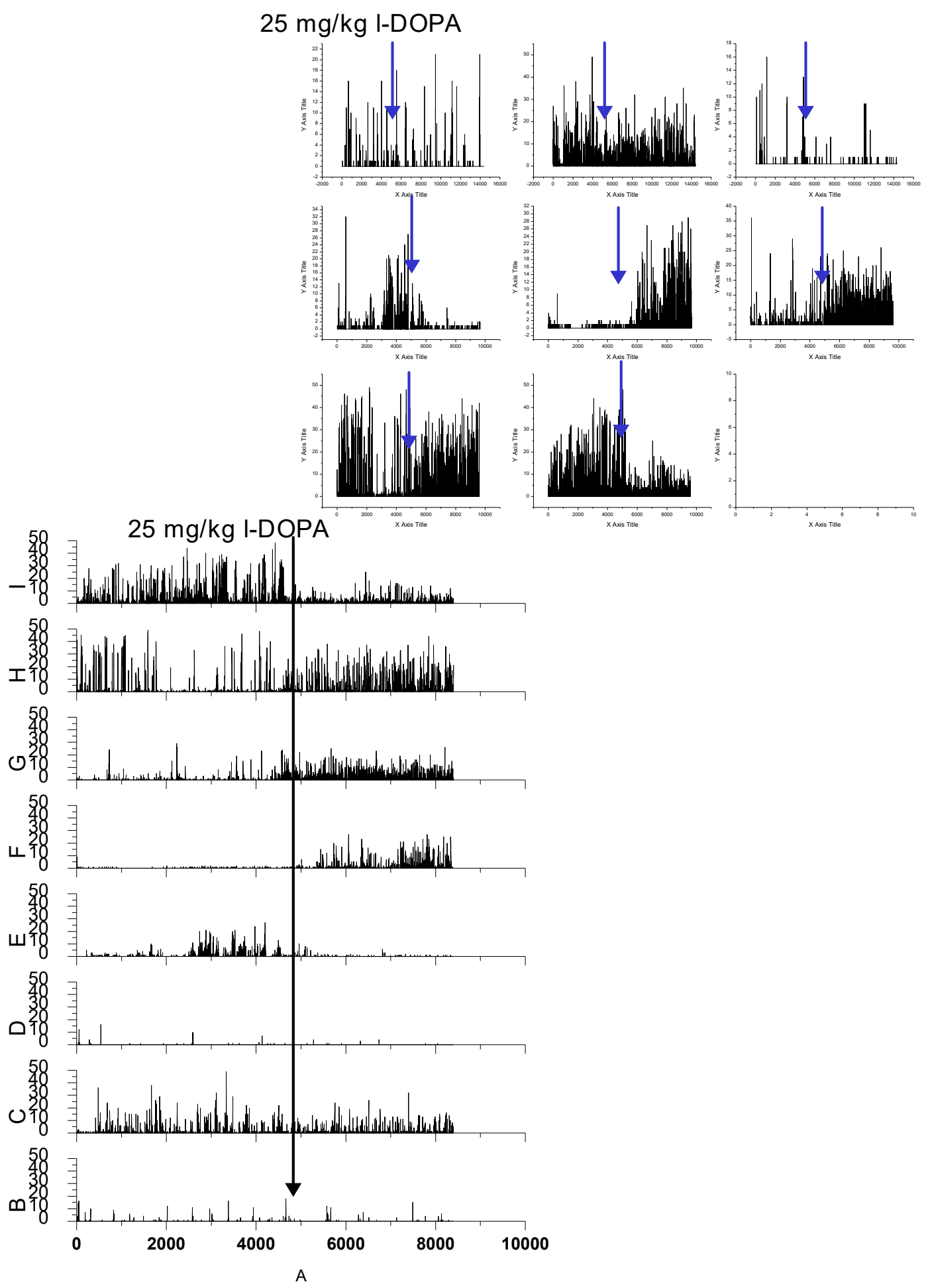

Figure 18. Exemplary Dorsal Pitx3Null Responses to l-DOPA.

Upper right - Injections shown using each cell's maximal duration and rate for $\mathrm{x}$ and $\mathrm{y}$ axes, respectively. Lower left - Same injections shown uniformly at $1800 \mathrm{sec}$ by clipping recording starts and ends; " $\mathrm{B}$ " here is upper left of upper right panel; panels are read across before down.) 


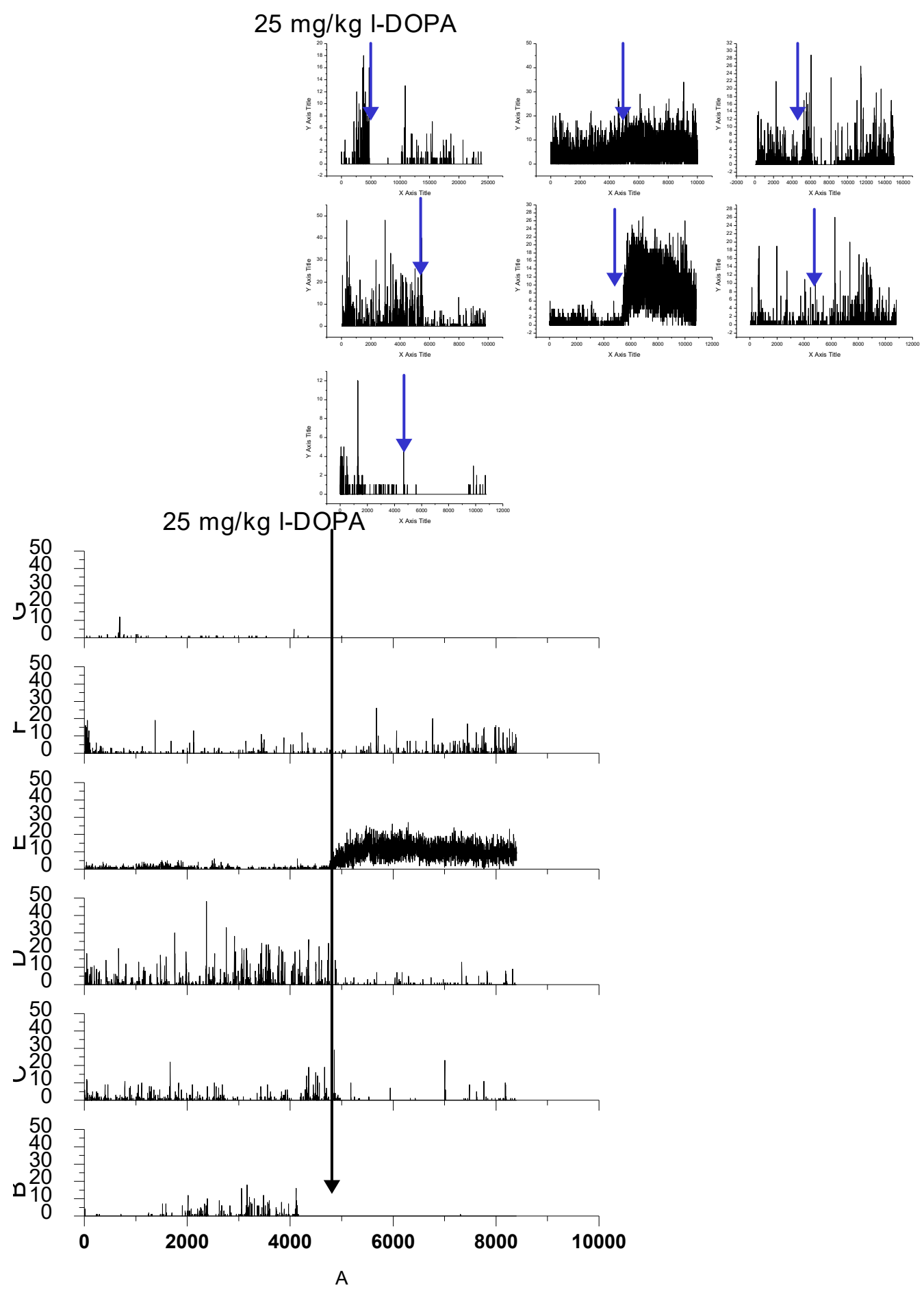

Figure 19. Exemplary Non-Dorsal Pitx3Null Responses to l-DOPA.

Upper right - Injections shown using each cell's maximal duration and rate for $\mathrm{x}$ and $\mathrm{y}-$ axes, respectively. Lower left - Same injections shown uniformly at $1800 \mathrm{sec}$ by clipping recording starts and ends; " $\mathrm{B}$ " here is upper left of upper right panel; panels are read across before down.) 


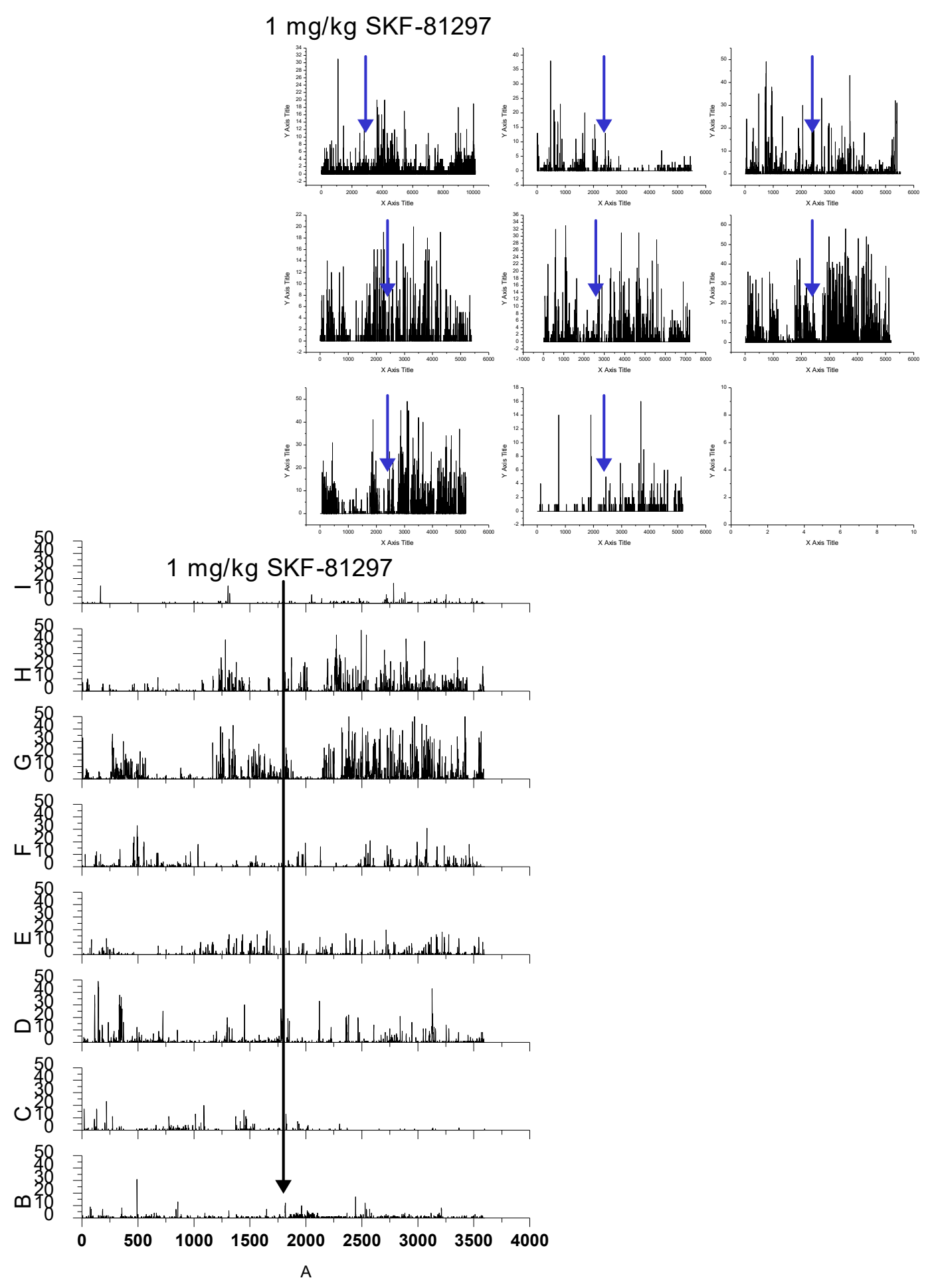

Figure 20. Exemplary Dorsal WT Responses to D1 Ag.

Upper right - Injections shown using each cell's maximal duration and rate for $\mathrm{x}$ and $\mathrm{y}$ axes, respectively. Lower left - Same injections shown uniformly at $1800 \mathrm{sec}$ by clipping recording starts and ends; " $\mathrm{B}$ " here is upper left of upper right panel; panels are read across before down.) 


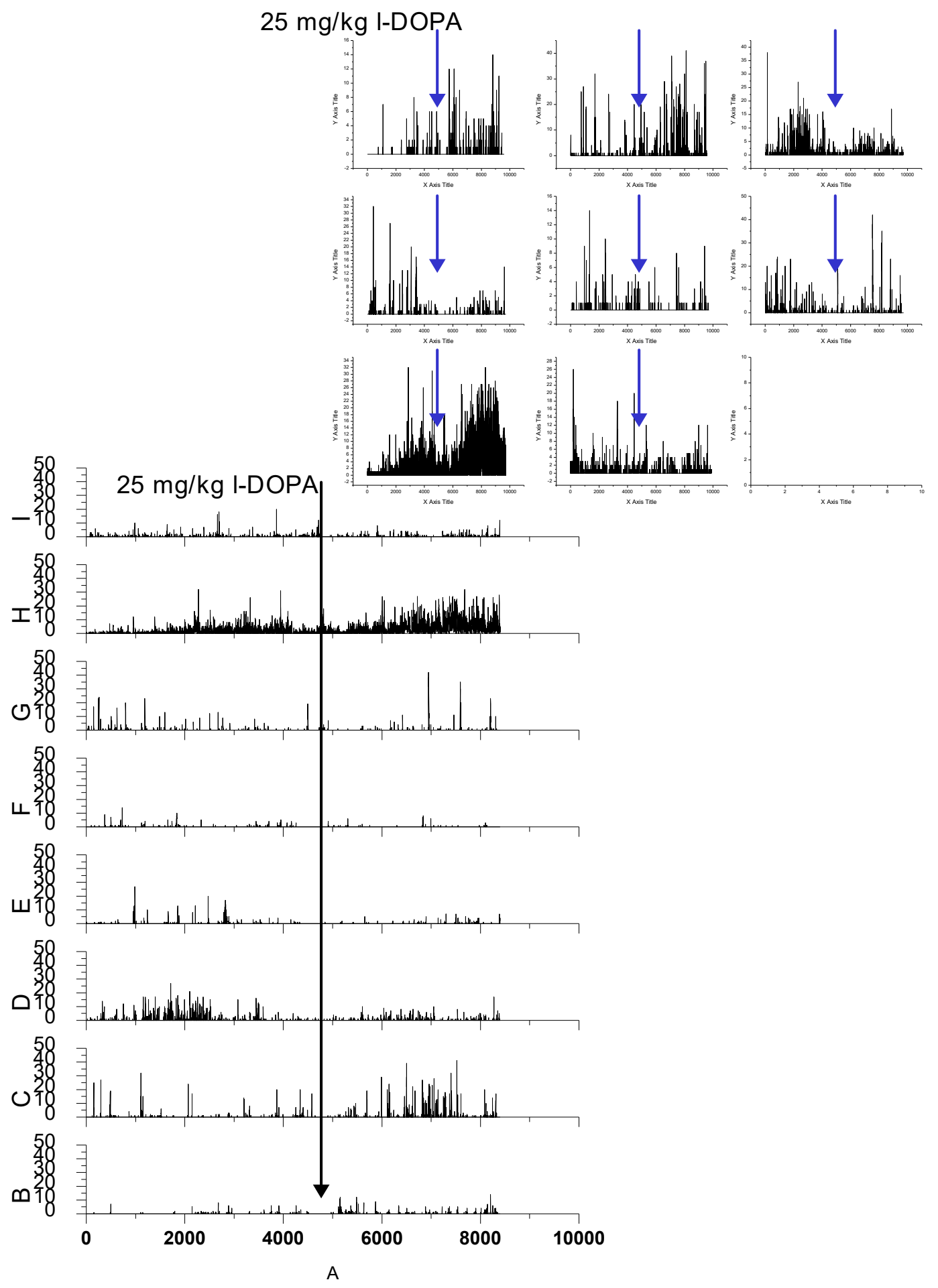

Figure 21. Exemplary Non-Dorsal WT Responses to l-DOPA.

Upper right - Injections shown using each cell's maximal duration and rate for $\mathrm{x}$ and $\mathrm{y}-$ axes, respectively. Lower left - Same injections shown uniformly at $1800 \mathrm{sec}$ by clipping recording starts and ends; " $\mathrm{B}$ " here is upper left of upper right panel; panels are read across before down.) 
test for such sporadic spiking as SPNs exhibit. The usual t-test for firing rate differences presumes a normal distribution of spikes per time binned in $\mathrm{x}$ second increments, the standard deviation of which is vulnerable to bias by varying the duration of each time bin, $\mathrm{x}$. Exploration of the effect of varying time bin duration left us with no obvious way to decide on an appropriate increment; thus a t-test procedure on its face did not seem to match SPN spiking patterns and lends itself to bias for which we could not account in order to resolve statistical from biological significance.

\section{l-DOPA response polarities}

Modeling the odds of a SPN increasing rate in response to 1-DOPA thus revealed a significant effect of baseline rate, which interacted significantly with depth (Table 4), but there was no significant effect of genotype. According to model estimates, upon injection of 1-DOPA, the odds of a positive response polarity were significantly lower with higher baselines, i.e., there was a significant negative correlation between baseline and the odds of a spiking-increase in response to 1-DOPA; specifically, according to the resulting baseline O.R., the average SPN having a tenth the baseline spiking of another had 3.261 times higher odds (Type III $p<0.0002$ ) of increasing spiking.

This significant negative correlation was significantly exaggerated in the dorsal striatum compared to non-dorsal regardless of genotype. The baseline O.R. specifically for the average dorsal SPN having a tenth the baseline of another was 7.40:1 (adjusted $p$ $=0.0010$ ). while that for non-dorsal was 1.44:1 (untested). These O.R.s were significantly different (depth-baseline O.R. $=5.14 *$, Type III $p=0.0082$ ), i.e., SPNs in dorsal striata had 7.4 times higher odds of increasing spiking, which odds were 5.10 $(7.40 / 1.44=5.10)$ times higher than non-dorsal odds of increasing spiking.

\section{D1 agonist ersponse polarities}

Upon injection of D1 agonist, the odds of a positive rate response polarity (i.e. a spiking increase) were significantly different in terms of a genotype-depth interaction and a 3-way interaction that included baseline in which (as with 1-DOPA) lower baselines predicted a greater chance of an increasing spike rate in response to drug, i.e. a negative correlation with baseline spiking.

Post-hoc comparisons of classes within the genotype-depth interaction (i.e. having set $\log _{10}$ (baseline) to various levels (spanning a half log unit on either side of the average baseline, $\sim-1.32--0.32$ ) in order to account for differences it made since it was involved in a significant 3-way interaction) revealed somewhat lower odds for Pitx3Null dorsal SPNs having baselines higher than average to increase activity than WT dorsal SPNs.

The other planned comparison was between depths in Pitx3Null mice, which gave $p>0.3$ for every baseline tested. Although not strictly tested during multiple comparisons, a depth gradient in WT was more evident at higher baselines than average (data not shown). 
Table 4. Overall Significant Results and Post-Hoc Comparisons for Both Drug Response Datasets.

\begin{tabular}{|c|c|c|c|c|}
\hline & \multicolumn{2}{|c|}{ I-DOPA } & \multicolumn{2}{|c|}{ SKF 81297} \\
\hline & polarity & intensity & polarity & intensity \\
\hline Genotype & $-0.69 x$ & $+0.43 *$ & -0.77 & +0.025 \\
\hline Depth & $-1.36 x$ & +0.07 & +1.16 & -0.055 \\
\hline Genotype * Depth & $\mathrm{N} \backslash \mathrm{A}$ & +0.27 & $-4.05 *$ & $\mathrm{~N} \backslash \mathrm{A}$ \\
\hline $\log _{10}($ baseline $)$ & $-3.3 x^{*}$ & $-0.27 *$ & +0.51 & $-0.35 *$ \\
\hline $\log _{10}$ (baseline) $*$ Depth & $-7.4 x^{*}$ & $\mathrm{~N} \backslash \mathrm{A}$ & $\mathrm{N} \backslash \mathrm{A}$ & $\mathrm{N} \backslash \mathrm{A}$ \\
\hline 3-way interaction & $\mathrm{N} \backslash \mathrm{A}$ & $-0.37 *$ & $32 x *$ & $0.27 *$ \\
\hline \multicolumn{5}{|l|}{$\begin{array}{l}\text { Planned and unplanned } \\
\text { comparisons }\end{array}$} \\
\hline WT depth interaction & $\mathrm{N} \backslash \mathrm{A}$ & Untested & $+24.1 \mathrm{x} * *$ & $\mathrm{~N} \backslash \mathrm{A}$ \\
\hline $\begin{array}{l}\log _{10}(\text { baseline }) \text { correlation } \\
\text { with Pitx } 3 \text { Null dorsal } \\
\text { intensities }\end{array}$ & $\mathrm{N} \backslash \mathrm{A}$ & -0.23 & $-4.7 x^{*}$ & $-0.51 *$ \\
\hline $\begin{array}{l}\text { Difference in } \\
\log _{10}(\text { baseline }) \text { correlation } \\
\text { made by genotype }\end{array}$ & $\mathrm{N} \backslash \mathrm{A}$ & +0.11 & $-6.9 x$ & $-0.35 *$ \\
\hline $\begin{array}{l}\text { Difference in } \\
\log _{10}(\text { baseline) correlation } \\
\text { made by depth }\end{array}$ & $\mathrm{N} \backslash \mathrm{A}$ & +0.15 & $-6.6 x *$ & -0.10 \\
\hline
\end{tabular}

$(*=\mathrm{p}<0.05$ adj. $)$

$(* *=\mathrm{p}<0.05$ unadjusted) 
Planned post-hoc testing of estimated differences between 3-way subgroups revealed that Pitx3Null dorsal SPNs showed a significant effect of baseline changes (Pitx3Null-dorsal-baseline O.R. $=4.69 *$, adjusted $\mathrm{p}<0.0471$, i.e. a unit decrease in these particular SPNs' baselines predicted significantly higher odds of a rate increase than decrease in response to Dlagonism); furthermore, this baseline effect was significantly exaggerated in the Pitx3Null dorsal location compared to non-dorsal SPNs (i.e. Pitx3Null-depth-baseline O.R. $=6.56: 1$, adjusted $\mathrm{p}<0.0464$, i.e. a stronger negative association with baseline dorsally).

Comparing Pitx3Null dorsal correlation with baseline to WT dorsal correlation with baseline indicated that genotype did not significantly alter dorsal-baseline O.R.s (genotype-dorsal-baseline O.R. $=6.874$, adjusted $p=0.0825$ ). However, accounting for the difference made by depth to the negative baseline correlation, then comparing genotypes (i.e. Pitx 3 Null depth effect on baseline correlation normalized to the same in WT) yielded an estimated genotype-depth-baseline O.R. $=32.63 *$ (TYPE III test $p=$ $0.013)$, i.e. the depth effect on the negative correlation with baseline was exaggerated significantly in the Pitx3Null DA gradient compared to WT, which has no such DA gradient (Figure 22).

Overall then, in terms of the 3-way interaction, the DA gradient in Pitx3Null animals was "top-heavy" (i.e. more negative baseline correlation for dorsal SPNs) in stark contrast to the "bottom-heavier" depth gradient expectation set by WT. Although statistical significance was not tested for (let alone reached) in most of these subgroups, this overall pattern of "different differences" made to the baseline correlations varying by depth and genotype is nonetheless significant as indicated by the Type III $p$ for 3-way interaction. Closer inspection of otherwise insignificant patterns in individual group estimates (at representative baselines, i.e. $+\backslash$ - a half $\log$ unit around the log mean, $\sim-0.8$ for both genotypes) suggested the tendency of even high baseline dorsal WT SPNs to increase spiking. This made for a much weaker baseline correlation whereas non-dorsal WT SPNs seemed not to correlate so weakly. Comparing this depth effect in WT (WTdepth-baseline O.R. $=0.201 \mathrm{x}$, unadjusted $\mathrm{p}=0.166)$ to that in Pitx 3 Null animals $(4.69 \mathrm{x})$ revealed a significant inversion (genotype-depth-baseline O.R. $=32.63 \mathrm{x}$ ) whereby Pitx3Null dorsal SPNs in particular maintained a significantly negative baseline correlation that was significantly stronger than that in non-dorsal, and significantly more so (dorsal stronger than non-dorsal) than WT; thus odds were greater for SPNs having lower rates to increase spiking (and higher for SPNs having higher rates to decrease) in dorsal Pitx 3 Null striatum than expected given the WT change in correlation with depth (and given the canonical rate model).

\section{Response intensity differences among WT and DA-deficient Pitx3Null mice}

In order to test the hypothesis that the DA denervation in Pitx3Null dorsal SPNs causes exaggerated spiking responses to DA agonism relative to dorsal WT and nondorsal Pitx3Null SPNs (having greater DA innervation), we recorded SPN responses to 1DOPA (25mg/kg with $5 \mathrm{mg} / \mathrm{kg}$ benserazide) or SKF $81297(1 \mathrm{mg} / \mathrm{kg})$ injections 


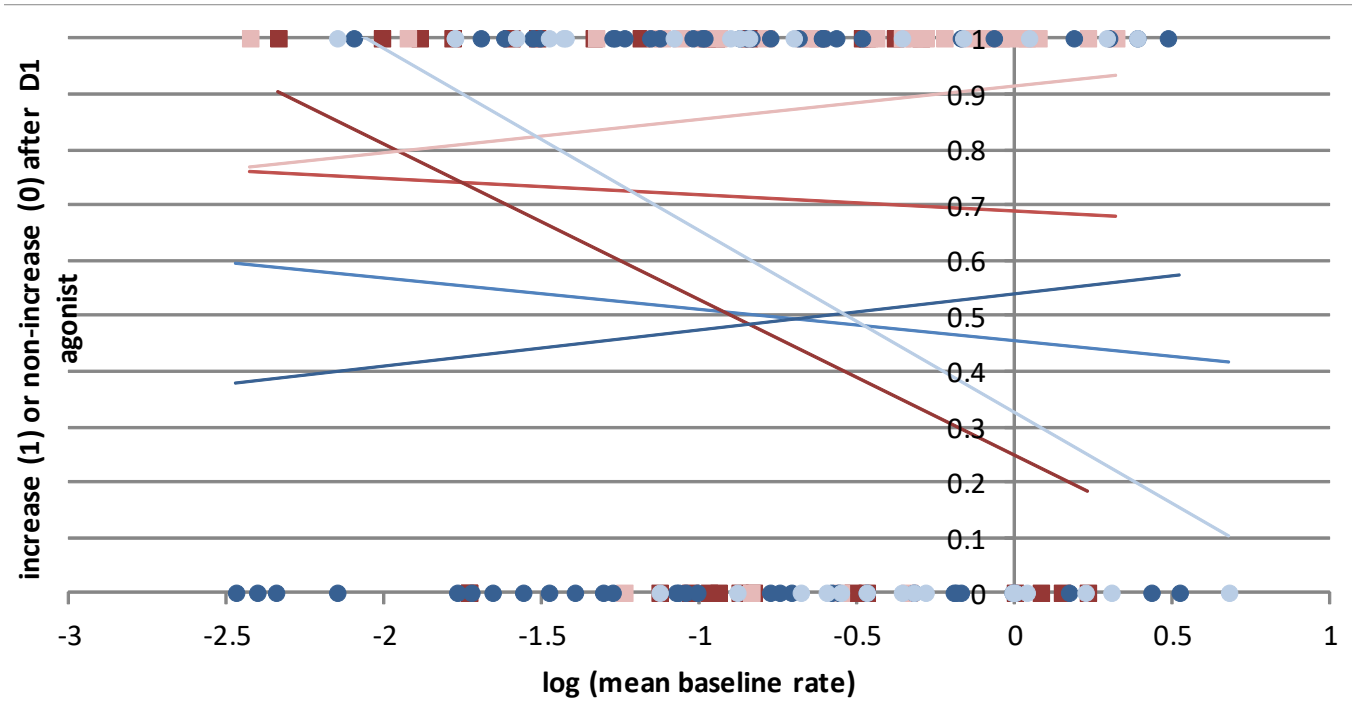

Figure 22. Response Polarity vs. Log Baseline.

The probabilities associated with these increasing relative to non-increasing responses were modeled and fitted separately for each class. Dorsal data is lighter than non-dorsal (in between is the mean for both, I.e. genotype mean); WT are cool colors (blue shades), Pitx3 Null are warm colors (red shades). $1=$ increase, $0=$ decrease. 
following baseline recordings. SPNs responded with varied intensities: from low $(<0.01$ $\mathrm{Hz}$ ) baseline rates having high rate responses $(\sim 1 \mathrm{~Hz})$ to vice versa and all combinations thereof. These responses were clear on both drugs in both depths from Pitx3Null animals, although generally appeared to be less intense in WT (Figures 18-21).

The absolute magnitude of the response intensity was taken so as to avoid using the overall mean of responses flanking (and thus tending toward) zero; also subsetting the responses into in-/decreasing would risk the generality of the model via post-hoc classification. Again, because the distribution of differences between responses and baselines spanned a similar range as the baselines (range $=0.00056-8.3 \mathrm{~Hz}$, i.e. $\sim 4$ orders of magnitude, Figure 23; data shown explicitly only for D1 agonist, but 1-DOPA responses were similar), the difference between $\log _{10}$ (response) and $\log _{10}$ (baseline) -to which we refer as the intensity- was used for the dependent variable in the model, We modelled the intensity as a function of our three factors of interest: $\log _{10}$ (baseline), depth, and genotype, plus interactions.

\section{l-DOPA response intensities}

Upon injection of 1-DOPA, both response polarities -i.e. absolute values of both increasing and decreasing $\log _{10}$ post/pre rate)- were more intense: $0.426 \log _{10}$ units according to modelled mean difference at $\log _{10}($ baseline $)=0$ (Type III $\left.p=0.0354\right)$ in Pitx3Null animals averaged across depth (a linear factor of about $2.7 \mathrm{x}$ more intense). They were also more intense the lower the baseline: $\left(0.269 \log _{10}\right.$ units per unit decrease in $\log _{10}$ (baseline) averaged across all classes. In linear units this is a factor of about $1.9 \mathrm{x}$ more intense.

Planned post-hoc tests for the significant 3-way interaction indicated that the slope of baseline in Pitx3Null dorsal striatum was not significantly non-zero after HolmSidak correction $(0.233$, adjusted $p=0.0635)$, was not significantly stronger than WT dorsal slope due to baseline (difference $=-0.101$, adjusted $\mathrm{p} \sim 0.5$ ), and was not significantly stronger than Pitx3Null non-dorsal striatal SPNs (-0.155, adjusted $p \sim 0.5)$. Exploring the remaining subgroups revealed that the slope of baseline in WT dorsal striata was likely non-zero $(0.335 \log$ units per $\log$ unit decrease in baseline, unadjusted $\mathrm{p}$ $<.0001$; this corresponds to a linear factor of about $2.2 \mathrm{x}$ more intense at a baseline $10 \%$ that of reference) and possibly higher than non-dorsal WT SPNs (0.213 log units, $1.63 \mathrm{x}$ linear factor unadjusted $\mathrm{p}<0.0382$ ). However, the difference made by depth to the baseline's effect (on response intensity) in Pitx3Null animals normalized to the same in WT was weaker within the Pitx3Null DA gradient: an estimated $-0.368 \log _{10}$ units per $\log _{10}$ unit baseline decrease (TYPE III test $\mathrm{p}=0.0477$ ), i.e. $\sim 43 \%$ that of WT.

These 3-way interaction results overall indicate an inversion of a slight depth effect in WT on the negative correlation of baseline with intensity; whereas Pitx3Null dorsal SPN baseline correlations compared to those of non-dorsal Pitx3Null SPNs were 

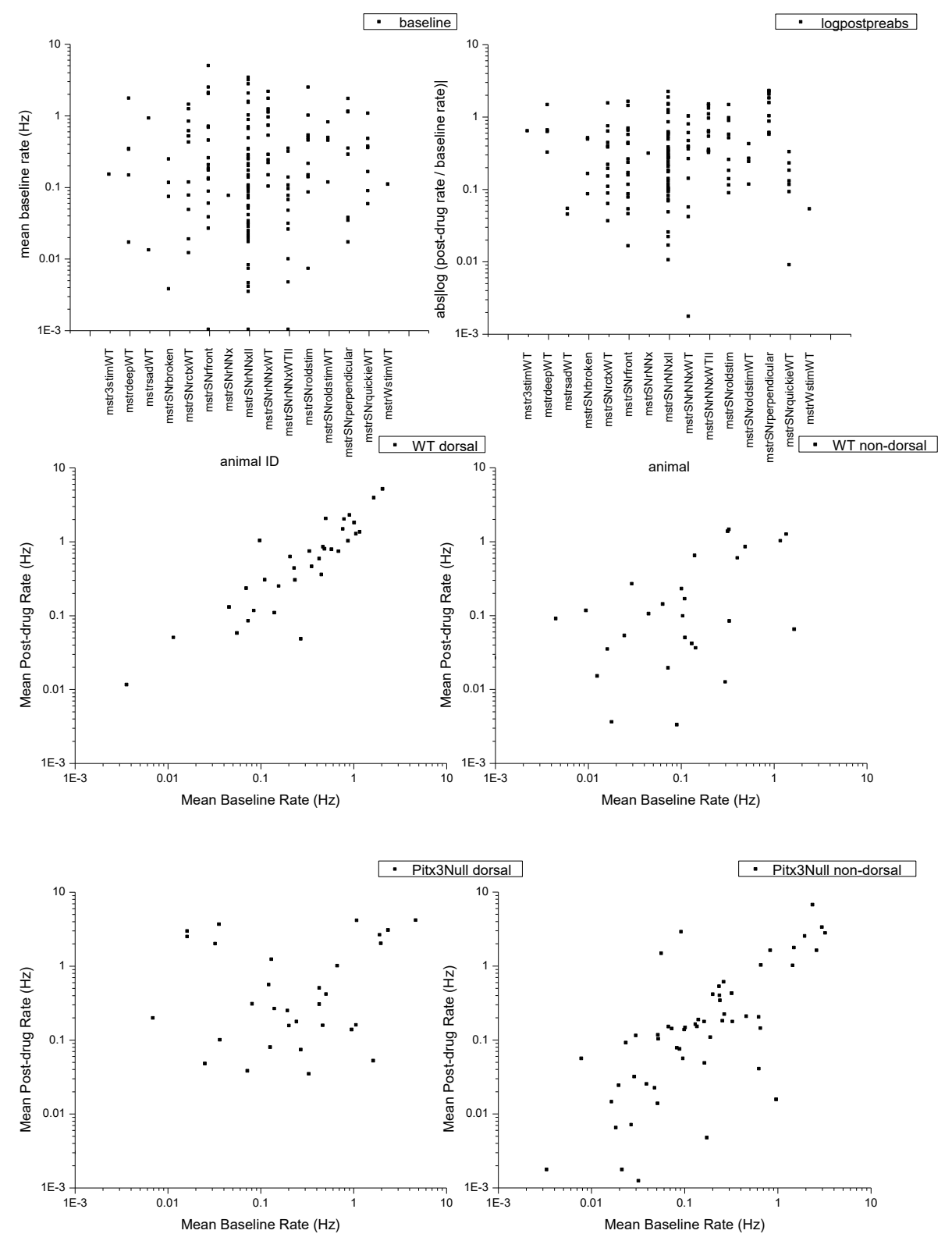

Figure 23. Mean Post-Drug Responses Are as Log-Distributed as the Baselines. Upper left - Mean baseline rates before D1 agonist ( $70 \%$ of all baseline data -partially overlapping approximately with that of 1-DOPA-, i.e. majority of baseline data and representative of entire dataset) are shown with respect to animal ID. Upper right response intensities to D1 agonism vs. animal ID. Middle left - Mean post-D1 agonist rate vs. mean baseline rate (log-scale) for WT dorsal cells. Middle right - as above for WT non-dorsal cells. Lower left - as above for Pitx3Null dorsal cells. Lower right - as above for Pitx3Null dorsal cells 
not significantly different, this difference was nonetheless significantly $\left(0.368 \log _{10}\right.$ units, a linear factor of $\sim 0.43 \mathrm{x}$ ) less than WT, where dorsal baseline correlations were likely the most significant and the depth effect on these correlations may or may not have been.

\section{D1 agonist response intensities}

Upon injection of D1 agonist, both response polarities (i.e. absolute values of both increases and decreases in spiking) were significantly different in a 3-way interaction of baseline rate, genotype and depth. Direct post-hoc pairwise difference estimates revealed that the significant negative correlation of baseline rate with response intensity $(0.345$ $\log _{10}$ units of intensity per $\log _{10}$ unit decrease in baseline averaged across all 4 classes, $p$ $<0.0001$, corresponding to $\sim 2.2$-fold increase at $10 \%$ baseline) was significantly stronger in Pitx 3 Null dorsal striatum $(0.507$, adjusted $\mathrm{p}<0.0001$, a linear factor of $\sim 3.2 \mathrm{x}$ stronger per $10 \%$ baseline) than WT dorsal (0.153): difference $=0.354 \log$ units, adjusted $\mathrm{p}=$ 0.0083, a linear factor of 2.26x in Pitx3Null). Pitx3Null dorsal striatum did not differ significantly from Pitx 3 Null non-dorsal striatum (difference $=0.103$, adjusted $\mathrm{p} \sim 0.4$ ). Although the depth effect on baseline correlations was inverted between genotypes, this effect was not significant; the Type III p-value represents the difference between genotype dorsal baseline correlations, i.e., a significant difference between at least two of the three subgroups (having already accounted for the doubly referential group, WT nondorsal.)

\section{Antidromically identified SPNs are deficient in baseline spiking and respond strongly to DA agonism}

Having been unable to use D1 agonism to differentiate satisfactorily between striatonigral and striatopallidal SPNs, and in order to potentially uncover a role of D1SPNs for the response intensity, they were putatively identified electrophysiologically in the hopes of finding them among those increasing activity (consistent with the canonical rate model) and to look for any evidence that their activity opposed expectations. Striatonigral (D1 receptor-expressing) SPN identification was accomplished using antidromic spikes elicited by substantia nigra stimulation (Ryan et al, 1989).

(Because this technique likely produces false negatives (Mallet et al, 2006) and because irresolvable cells responding to stimulation may confound interpretation, commentary concerning the subset of identified cells is restricted to those that responded unambiguously - i.e., better isolated than most and with minimal overlap from coresponding units- to nigral stimulation and is not necessarily representative of the entire population of striatonigral SPNs.) Antidromically identified SPN baselines (all dorsal) include a total of 6 SPNs from 3 Pitx3Null animals and 8 SPNs from 3 WT animals. Nonparametric statistics for these data are shown in Table 5. The antidromically identified 
Table 5. Antidromically Identified Cell Baselines Between Genotype and Depth.

\begin{tabular}{cccccccc}
\hline Genotype & Depth & $\begin{array}{c}\text { N } \\
\text { Obs }\end{array}$ & Minimum & $\begin{array}{c}\text { Lower } \\
\text { Quartile }\end{array}$ & Median & $\begin{array}{c}\text { Upper } \\
\text { Quartile }\end{array}$ & Maximum \\
\hline Pitx3Null & dorsal & 6 & 0.01976 & 0.02027 & 0.02640 & 0.08764 & 0.08764 \\
WT & dorsal & 8 & 0.01183 & 0.20093 & 0.31922 & 0.46390 & 5.94051 \\
\hline
\end{tabular}


cell baselines were found to be significantly lower (on average -0.937 log units +- - std err $0.297, \mathrm{p}<0.05)$ in Pitx3Null animals than WT. In linear units this corresponds to Pitx $\left(10^{-1.486+/-0.111}=\right) 0.0327 \mathrm{~Hz}+0.0095 /-0.0074$ and WT $\left(10^{-0.549+/-0.276}=\right) 0.282 \mathrm{~Hz}+0.251 /-$ 0.132 yielding a log difference (aka linear factor) of between $5.83-22.9 \%$ of WT centered on $11.6 \%$ (i.e. $10^{-0.937+/-0.297}$ ).

Furthermore, no identified Pitx3Null cells decreased activity in response to either drug; this made odds infinite with respect to WT decreases and thus precluded polarity analyses. Figure 24.

Finally, the response intensities were all higher for dorsal Pitx3Null cells than WT. However, given the small sample size in the antidromically identified subset, t-tests were used in order to avoid overcomplicating the models for both drugs with considerations known to be important in previous models (e.g. intra-animal correlation of responses); thus assuming SPN independence allows us to show a preliminarily significant difference between genotypes in both datasets best seen in Figure 25 as all dorsal Pitx3Null striatonigral cells had higher firing rate response intensities than WT striatonigral cells.

\section{Results for Fibers of Passage Mistaken as FSIs}

During in vivo electrophysiology experiments for a different project (Sagot and Zhou, submitted), antidromic identification of direct pathway SPNs yielded occasional responses at much shorter latency than expected as seen in Figure 14 (Ryan et al 1986, and 1989) and of much shorter waveform duration than expected for SPNs (Mallet, 2005); compare units in Figure 14.

Four such units were collected and their identification metrics and initial responses are presented by row in Figure 26; they demonstrated all-or-none -as opposed to graded-- responses to increasing current intensity and consistently followed the stimulus at precise latencies $0.5-1.63 \mathrm{~ms}$ (implying conduction velocities from $\sim 2.7-10$ $\mathrm{m} / \mathrm{sec}$ ). For three of the four units in question, responses to further stimulation patterns were collected in order to confirm a refractory period $<2.0 \mathrm{msec}$ during which the second of two stimuli would not elicit a response. (Figure 26, two right-most columns) (The second unit from top was not ignored but accidentally discovered while searching in an otherwise uneventful recording, during which stimulations were abandoned due to the lack of SPN responses.)

Altogether the pattern of responses for these units suggested an antidromic nature, so their recordings were scrutinized for evidence of random collisions, collisions being the gold standard identifier of antidromic spikes. Notwithstanding hardware shortcomings for trigger latencies (the analog Omniplex system), we occasionally stimulated soon 

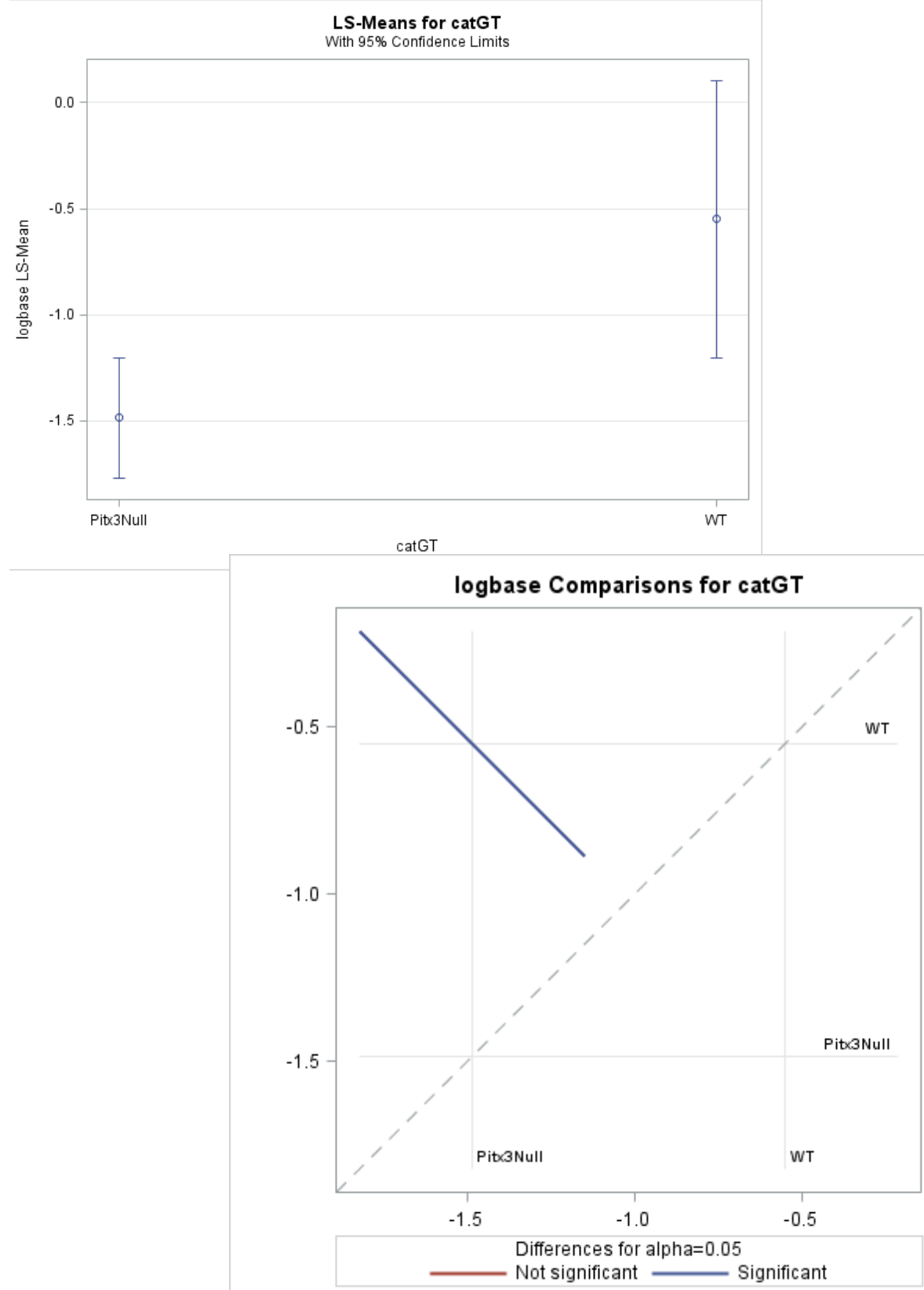

Figure 24. Baseline Data (Least-Squared Means) for Putative D1 Cells.

Top panel: modeled means $+\backslash$ - respective standard errors for each group bottom panel: diffogram showing how far the difference between genotype baselines is from equal $(+/-$ standard error). catGT $=$ genotype, $\log$ base $=\log 10($ baseline $)$. 

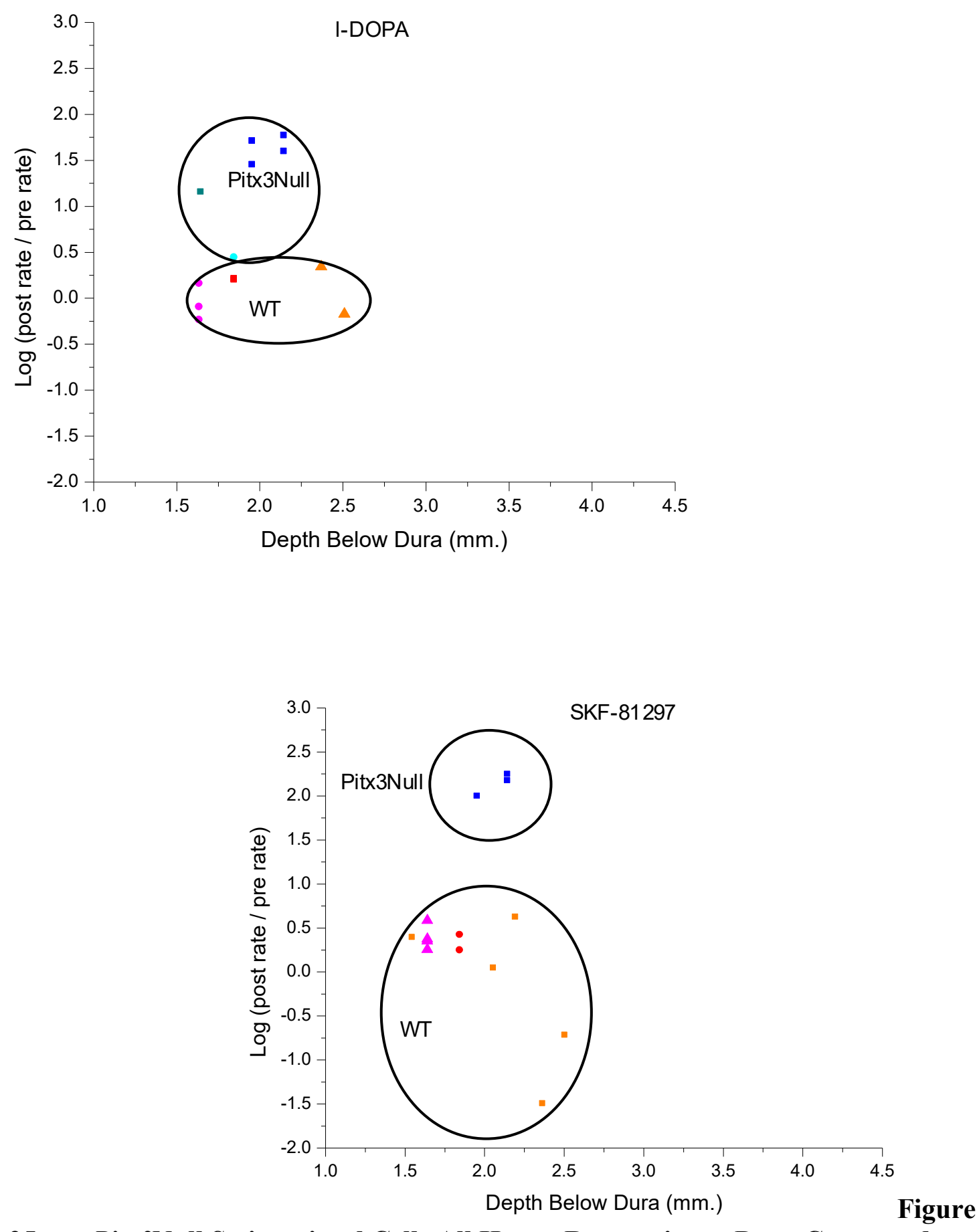

25. Pitx3Null Striatonigral Cells All Hyper-Responsive to Drug Compared to WT.

Response intensity (not absolute value) vs. depth below dura shows the distinctions between genotypes for striatonigral SPN responses to both drugs: 1-DOPA left, D1 agonist right. (Note lowest two cells were not included in calculations due to non-dorsal depth.) 
Figure 26. All Responding UnitsLeft-most column - average (+/- std) of waveforms isolated from the respective unit's in question. Middle left column - To the immediate right are their respective ISI histograms and rate over time plots. Middle right column Stimulating (train of two stimuli separated by $100 \mathrm{msec}$ ) around threshold current yields a stimulus artifact without the putative antidromic spike (respective to each unit's row) in the upper panels of this column. In the lower panels, the spike is reliably present after increasing the stimulating current.

Right most column - Upper sub-panels in this column show (in each respective unit's row) nigral stimuli $2.0-2.5 \mathrm{msec}$. apart eliciting two corresponding spike responses yielding information regarding the putative antidromic spike's ability to follow high frequency stimulation. Setting the interstimulus interval to $<1 \mathrm{msec}$ in order to show only single responses to two stimuli (in the lower sub-panels of this column) yields information regarding the putative antidromic spike's refractory period, the presence of which excludes the possibility that the spike response is simply part of a stimulation artifact. 

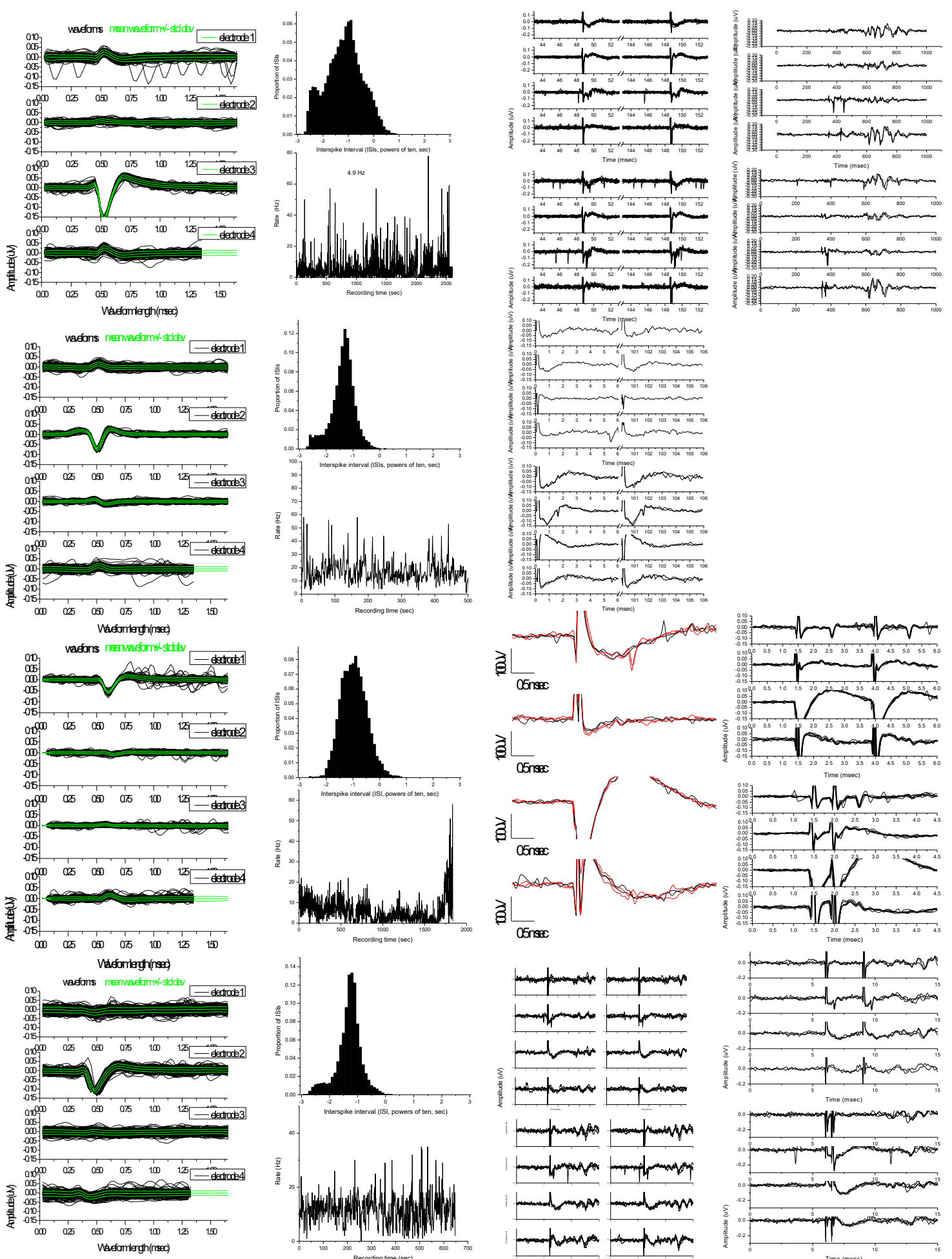

है
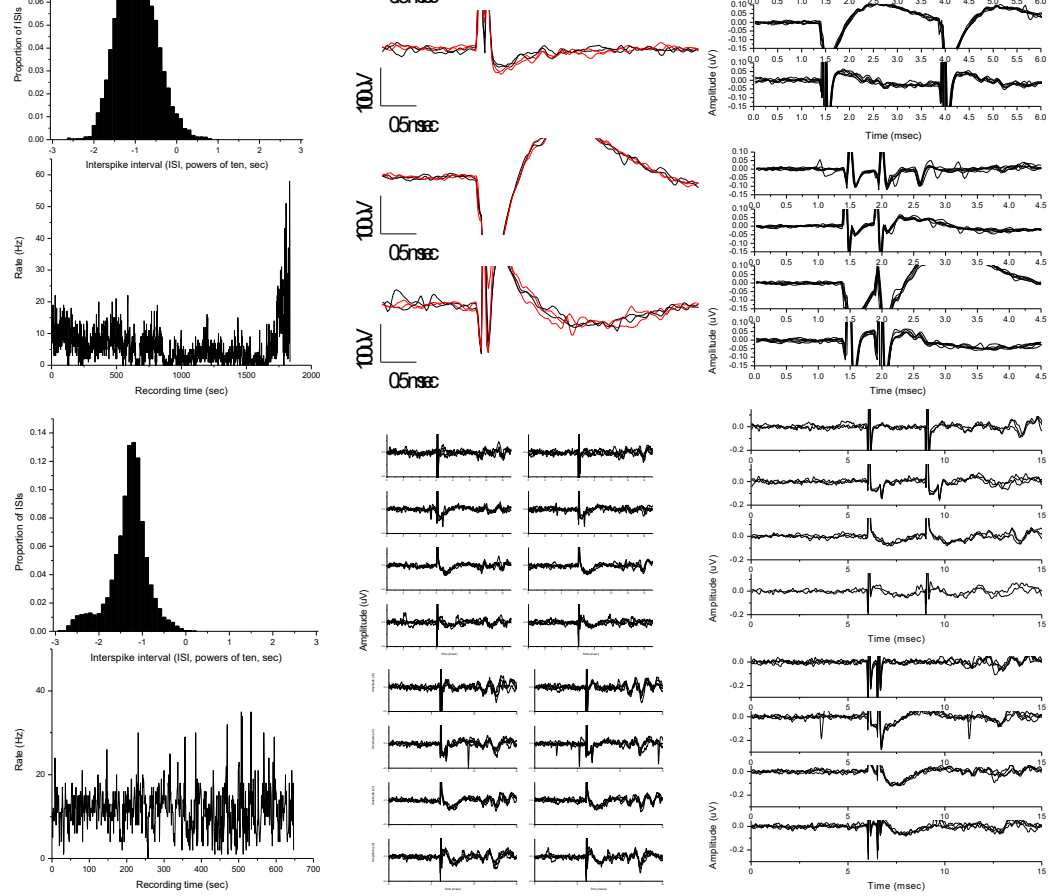
enough after spontaneous spikes to discover such evidence. Collisions are shown in Figure 27 (and may be compared to those of a nighboring SPN from Fig. 5, upper unit's trace panel). One putative fiber from each animal was occasionally interrupted by what appeared to be the same waveform on the same channel, i.e. unexpectedly absent within msec's after a similar spike when otherwise present on flanking stimuli, and thus strongly reminiscent of an antidromic collision. (Swadlow et al, 1978)

Given the low density of FFNs in the striatum (Luk and Sadikot, 2001, Saka et al, 2002) and no published knowledge of their tendency to cluster, it is very unexpected to find that more than one may be recorded simultaneously, yet exactly such data has been reported (Berke, 2008), and indeed all four neurons in this report were accompanied by at least one (incompletely recovered) and up to four units (two completely recovered) having similarly brief waveforms. (Figures 28) Units lacking waveforms due to incomplete recovery ( $\sim 20-50 \%$ false negatives $)$ gave no rate or ISI metrics; however, all had the characteristic brief waveform.

All units had a full width at half-max of $0.08-0.18 \mathrm{msec}$ (compared to SPNs having $0.15-0.35$-Figures 26 and 27, left-most columns and Figure 27 right units, SPNs), latency to response of $<2 \mathrm{msec}$, and one unit from each animal was interrupted multiple times by spontaneous spikes. Mean rates varied between $\sim 5-17 \mathrm{~Hz}$, and the units recorded during quiet rest, and motor activity switched to a bursty mode with long pauses reminiscent of reported FSIs (data not shown). (Berke, 2011) 
Figure 27. All CollisionsLeft unit panels - Topmost panel shows (after establishing the latency to response for this spike in the $1.1-1.3 \mathrm{msec}$ range) that this first response is early enough to be considered spontaneous; no response follows, but the next response is appropriately timed. The middle panel shows a spontaneous spike in the second stimulus artifact (note exaggerated downward deflection compared to all others during that bout of stimulation (exemplified by the first stimulus and thosein the upper panel); even though flanking stimuli produced responses, this one did not. The lower panel shows that the fourth stimulation (red trace) in a train of four spaced $10 \mathrm{msec}$ apart was preceded ( 100 usec) by a spontaneous (orthodromic) spike; even though flanking stimuli produced typical spike responses (first 3 black traces, next train not shown), this one did not. Right unit panels - Stimuli artifacts (on all four electrodes, shown vertically) are shown flanking the stimulus of interest, on right of upper panel and left of middle panel. Multiple stimuli are overlaid and colored in these graphs. Spontaneous spikes from the unit of interest precede the stimuli of interest by $0-1 \mathrm{msec}$; note completely nonresponsive antidromic spikes, putative collisions Lower left panel Traces from 49 stimuli at increased current having $100 \mathrm{msec}$ interstimulus interval are overlaid; note unit most prominent on third electrode responding to all but two stimuli for each of which a spontaneous orthodromic spike apparently precluded the antidromic response; lowest left panel shows close-up of stimuli, responses, and putative collisions of interest for the same data. 

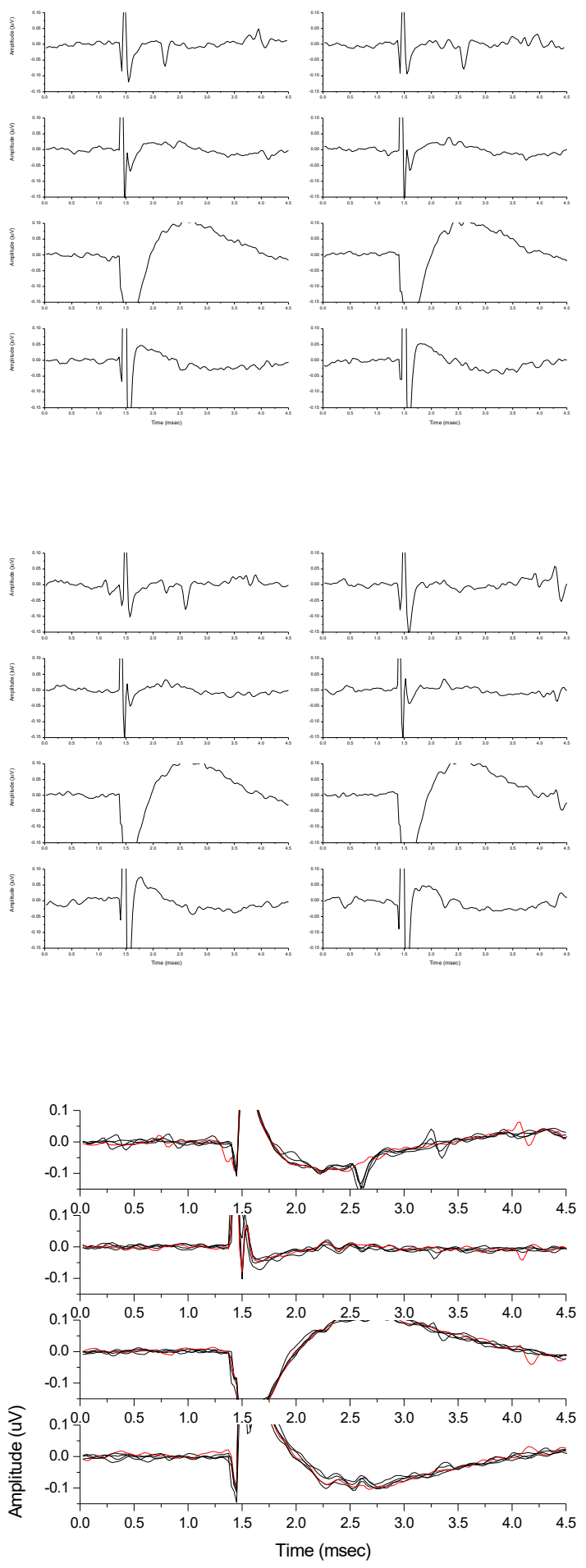
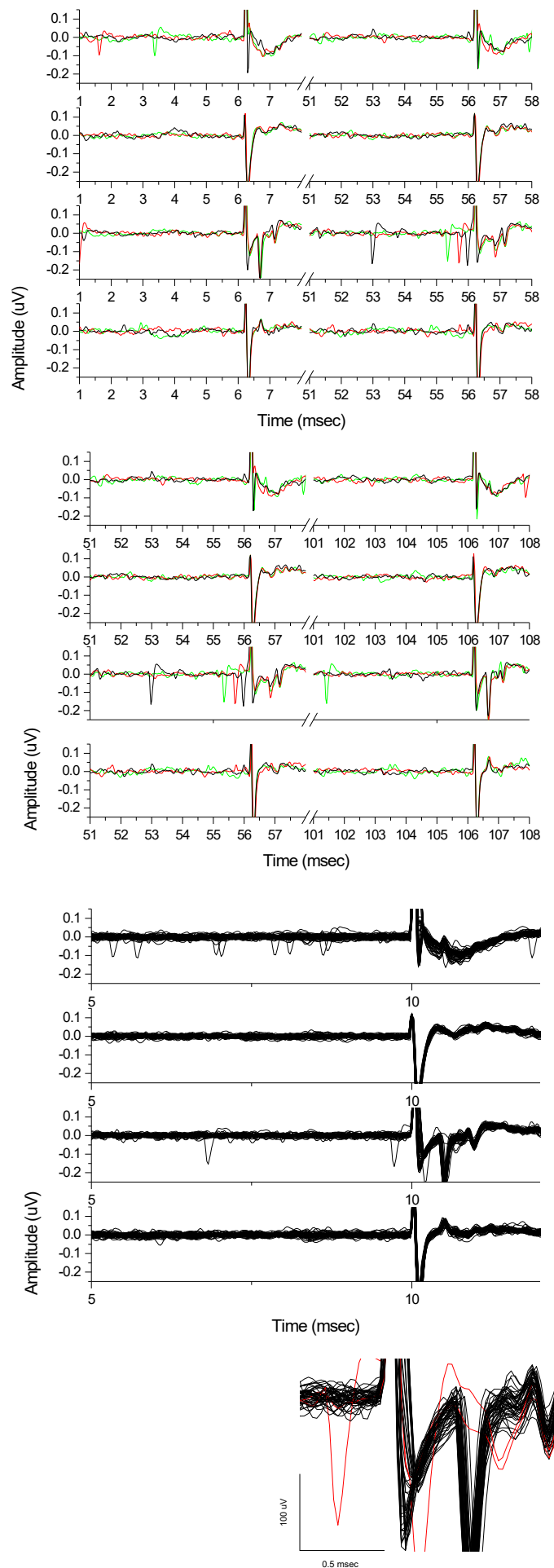
Figure 28. All Neighboring Units and Two Co-responding SPNsAll units on left (and corresponding identification metrics to their immediate right) were recorded simultaneously with the respective responding units from Figure 27. (Only the secondunit from top of Figure 27 is missing a neighbor due to incomplete recovery; in its place is another neighbor from the recording of the 3rd unit from top.) Upper right unit - The mean waveform +/- standard deviation of a responding SPN previously recorded is shown. ISI histogram of the unit showcasing log normal distribution biased towards) longer pauses. In the panel just below a collision between the same unit's antidromic spike in response to the first of four stimuli is not evident (left half), theoretically due to the spontaneous spike's lagging the stimulus by enough time to allow it to pass the stimulated portion of axon before an antidromic spike is elicited there; however, the next spontaneous spike (right half) precludes the antidromic response in the first of the latter four stimuli, theoretically due to collision of the two between the point of recording (striatum) and the point of stimulation on the cell's axon ( $\mathrm{SNr}$ ).

Lower right unit - The mean waveform +/- standard deviation of a putatively responding, co-recorded SPN is shown to left of ISI histogram showcasing log normal distribution biased towards longer pauses. In the lowest right panel putative SPNs responded at lower thresholds than the unit of interest, as seen most prominently in the first electrode (and less so on the fourth). 


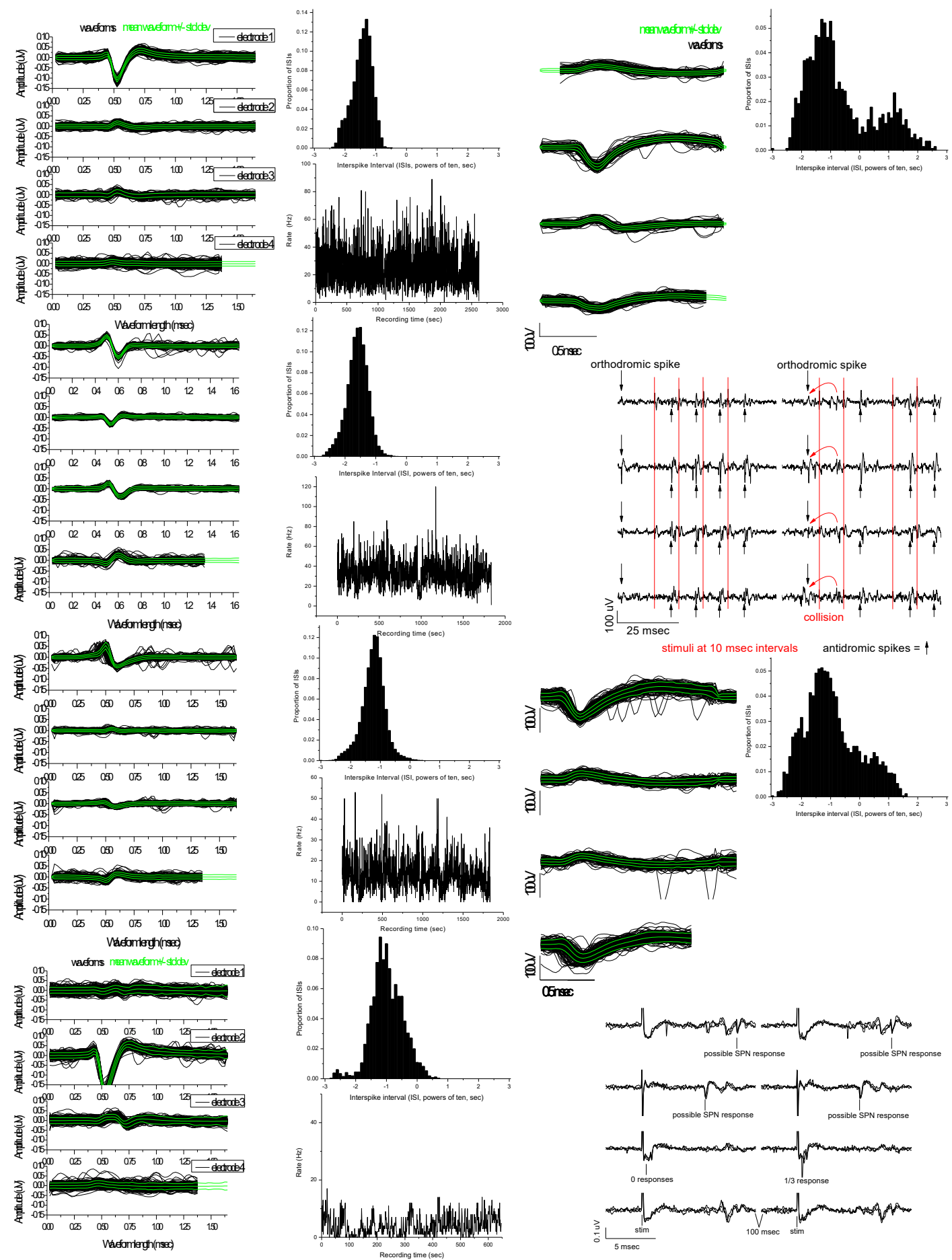




\section{CHAPTER 5. DISCUSSIONS}

\section{Discussion for DA Agonism in DA-Deficient SPNs}

\section{Interpretation within the Canonical Rate Model}

In this paper, we demonstrate some long-term effects of DA denervation on SPN spiking responses to acute DA agonism: namely, a more drastic rate change in response to DA agonism among SPN populations having low baseline spiking and lor located in the DA-denervated Pitx3Null dorsal striatum However, this may not be a direct receptor influence on spiking since it was found in both dorsal, DA-denervated, and non-dorsal, having residual DA, Pitx 3 Null striatal regions (see further discussion on depth effect). We interpret these results on the basis of a modified understanding of the canonical rate model allowing for coactivation of striatonigral and striatopallidal cells during behavior (e.g. Sippy et al., 2015, Cui et al., 2013) as well as for network effects that may overwhelm the effects of DA receptor stimulation on spiking (Ruskin et al., 1999, Nambu, 2008, Beeler et al., 2012, Friend \& Kravitz, 2014). Given the supersensitized DA receptors in DA denervated Pitx3Null dorsal SPNs, we thus expected two primary effects of DA agonism: stronger spike-rate intensities in Pitx3Null dorsal than Pitx3Null nondorsal SPNs and than WT dorsal SPNs. Our interest in polarity was exploratory inasmuch as modifications to the canonical rate model muddy the clarity of its polarity predictions.

In testing for significant group differences and effect sizes in our factors of interest, two notable patterns emerged; our predictions were upheld in genotype but not depth differences, and baseline spiking had a significant (usually main) effect across the entire dataset. Including baseline as a covariate was intended to account for possible floor or ceiling effects on rate changes given baseline spiking (e.g. increasing spiking from a high baseline is necessarily less of an effect than doing so from a low baseline).

Furthermore, the higher odds of SPNs increasing activity if they have lower baselines combined with the same correlation for intensity implies that increases tended to be more intense. This may have implications for DA's effects on striatal spike coding of behaviour.

\section{Genotype differences}

WT mice placed into an open field and given a behaviorally inert drug, 1-DOPA (Kim et al., 2000, even after slight DA depletion Costa et al., 2006, although see Huot, 2015 for a comprehensive review), yield spiking responses used as a control for this dataset, reflecting background neuronal processes and/or any changes in open-field behavior-related spike-firing as the session runs its course. WT responses to $\sim 2$ fold extracellular [DA] increase (Abercrombie et al., 1990) are expected to provide the control against which presumptively vigorous SPN receptor responses to 1-DOPA in Pitx3Null yield spiking changes more intense than WT but with a nonetheless similar ratio of 
putatively D1-based excitatory and D2-based inhibitory responses. Bearing this in mind, then interpreting the pattern of baseline correlations (at any level or group) with the dependent variables requires knowledge of the "normal", i.e. WT, dorsal striatal correlation of baseline with the dependent variable. Thus expected differences between subgroups in the baseline correlations become a central theme in discussing complex results.

These expectations change somewhat for D1 agonist, SKF 81297. While the authors know of no awake WT data correlating baseline differences with response differences, WT locomotive activity is known to increase significantly only at doses 0.5-3 times that used here (Xu et al., 1997, Usiello et al., 2000, Kim et al., 2000), although overall changes in SPN spiking are less straightforward. For example, adding SKF-81297 to the iontophoretic co-application of glutamate and a D2 agonist in anesthetized mouse ventral striatum inhibited the glutamate-induced $\sim 4-5 \mathrm{~Hz}$ baseline firing rate (relatively high considering our dataset range, after $\log _{10}$ transform, of $\sim-3-+1$, mean $\sim-0.8$ ) (Xu et al., 1997). In another example, strict application of SKF-81297 in a similar setting for dorsal striatum showed no obvious correlation with baseline for polarity or intensity. (Xu et al., 2011) Finally, Delfino et al., 2007, found that motor cortical activity was enhanced in hemi-lesioned rats having their BOLD response measured, suggesting that motor network glutamatergic striatal input plays a larger role in the D1 agonist response than in the 1-DOPA response for WT. Thus expectations are that high-rate non-dorsal SPNs in WT may tend to decrease in rate albeit after drug during locomotor increases, significant or not, and any dorsal baseline correlation should be weak, if present at all.

Genotype differences arose at four points in our study; most noticeably in the much lower baseline spiking from antidromically identified cells, but also in the 1-DOPA and D1 agonist intensities as well as Dlagonist response polarities. In the 1-DOPA dataset this manifested as a main effect of genotype as well as a complicated interaction of genotype depth and baseline. A similar interaction held in the D1 agonist response polarities. Finally, a genotype difference in D1 agonist response intensities was evident in the dorsal subgroups.

\section{Baselines}

The baseline difference in antidromically identified SPN spiking between the two genotypes is interpreted as a direct consequence of DA denervation in the Pitx3Null animal. The only manipulation in this experimental system is that of lost ventral tier SNc DA neurons (and aphakia, which was shown to allow similar rearing behavior in blinded versus intact WT controls in Kas et al., 2008); thus, the most parsimonious explanation for the baseline spike difference in the brain is the striking DA loss there.

This result is particularly important in light of the baseline behavioral phenotype in Pitx3Null mice, These mice have less spontaneous rearing, take longer and more steps to traverse the challenging beam, take longer to orient downward in the pole test (Hwang et al., 2005), take longer to remove adhesive from snout (Fleming et al., 2013). take less 
time to fall from the rotarod (Beeler et al., 2009), more time to traverse the balance beam, more time to complete the string test, and shred less cotton than WT (Singh et al., 2007). These are consistent with canonical rate model and the behavioral effects of deficient DA agonism on striatonigral SNr afferents. (Rommelfanger \& Wichmann, 2010)

\section{l-DOPA responses}

1-DOPA's effect on SPNs' spiking was clearly more intense in Pitx3Null than WT mice $(0.426 \log$ units $=2.67 *$ higher on average $)$, which validates our prediction that Pitx3Null SPNs are hyper-responsive to DA agonism compared to WT. Publications quantifying spike-rate response intensities to l-DOPA in freely-moving WT mice are not known to us, therefore we can only speculate that given their lack of behavioral response, their spiking responses would be expected - at the crudest level - to similarly lack intensity.

The similar extent to which WT dorsal and Pitx3Null dorsal SPN baselines were negatively correlated with spiking change intensities to 1-DOPA may be expected if the normal WT reduction in motor behavior in the open field over periods longer than minutes (Costa et al., 2006) corresponds to any grossly similar phenomenon reflected in SPN spiking. Since baseline was negatively correlated with intensity for both datasets, this may reflect a normal condition of SPN spiking changes (most strongly suggested by the WT dorsal negative baseline correlation on 1-DOPA, unadjusted $\mathrm{p}<0.0001$ ).

Furthermore, the significant 3-way interaction in the 1-DOPA intensity dataset implies a "top-heavy" WT depth differential in terms of baseline correlations that in the Pitx3Null animals is significantly less different. Our explanation for such an unexpectedly complicated finding is only speculative. That a lower baseline will tend (whether or not significantly and even under drug-free or low-dose conditions) to have a stronger response and vice versa (higher baseline having less response) may be expected to differ between WT depths (again, whether or not significantly); although any openfield electrophysiological responses to 1-DOPA in WT mice are not known by this author in the literature, sensorimotor (i.e. dorsal, West, 1998) SPN spiking intensity reversing over time in the open field in WT as their exploratory behavior subsides might be one way this could happen. This "top-heavy" difference contrasted greatly with the Pitx3Null depth difference wherein non-dorsal responses more closely matched dorsal responses,

The weaker depth difference in Pitx3Null mice evident in the significant 3-way interaction may indicate a few possibilities. Since both Pitx3Null and WT non-dorsal striata have some or full DA innervation, their different differences with respect to dorsal striatum likelier involves some non-DA-ergic effect above and beyond or even instead of supersensitive receptor stimulation; e.g. regardless of the origin of hyper-responsive SPNs (without being able to rule out non-dorsal SPNs as the initiators), the overall effects of DA deficiency on the negative SPN spiking correlation with baseline are reflected throughout the striatum. E.g. it could be the result of altered motor network processes recruiting otherwise quieter non-dorsal striatal regions once a drug effect has begun (as in 
striatonigrostriatal loops in primates shown anatomically by Haber et al., 2000, or the split circuit hypothesis advanced by Joel \& Weiner, 1994, also see Beeler et al., 2012 for network simulation, and Beeler et al., 2008 for Pitx3Null relevant results). Another possibility could be a developmental compensation, as slight as the non-dorsal DA loss, of the non-dorsal baseline correlation, ensuring more responsive SPNs (notwithstanding dorsal Pitx3Null motor effects of DA denervation being independent from development as shown in Li et al., 2015). Similarly any non-dorsal compensatory change (developmental or otherwise) may have an overall network effect that reinforces the baseline correlation in individual SPNs (although see Bamford et al., 2004) leading to the overall pattern observed.

\section{D1 agonist, SKF 81297}

Similarly parsing the significant 3 way interaction from D1 agonist response polarities, the WT tendency for a dorsoventral gradient of negative baseline correlation with rate increases to 1-DOPA was significantly inverted in Pitx 3 Null mice. This may be evidence that lacking DA in the Pitx3Null dorsal striatum allows higher baseline SPNs to pathologically decrease activity in response to DA stimulation and lor makes some less direct contribution to reduce the likelihood (albeit possibly insignificantly on its own) of an increase in rate in the non-dorsal region.

Also noteworthy in the D1 agonist polarity results is the genotype-depth interaction independent of baseline: a striking ubiquity of WT dorsal rate increases in response to a selective D1 agonist, SKF-81297. In contrast, Pitx3Null SPNs tended to decrease rate (approaching $\mathrm{p}=0.05$ ) in response to D1 agonism. This is weak evidence for D1 agonism supporting a rate decreasing role in DA-deficient SPNs. (Johansen et al., 1991, Xu et al., 1997, Fienberg, 1998)

This assertion is further supported by the 3-way interaction (i.e. including baseline as a covariate. Pitx3Null dorsal SPN response polarities to D1 agonism may be more sensitive to their baseline rates than those of WT SPNs given the $\sim 7$-fold stronger negative correlation (notwithstanding its own statistical insignificance) where acute D1 agonism reached SPNs in an otherwise DA deficient context. This is supported by the significant Pitx3Null dorsal baseline correlation significantly stronger than non-dorsal (discussed below), which pattern was significantly inverted between genotypes (discussed above). Thus a preponderance of evidence supports a role for DA deficiency in dorsal SPN spiking decreasing in response to acute D1 specific agonism in behaving mice as has been shown in other preparations and species (anesthetized Ballion et al., 2009, Tseng et al., 2004, and in vitro Nisenbaum et al., 1992, Johansen et al., 1991), especially if they have low baseline spiking.

A WT control situation for response intensities to D1 agonism is expected to be different from that to 1-DOPA. WT animals are known to respond to SKF-81297 with increases in locomotor activity (Xu et al., 1994, Xu et al., 1997, Usiello te al., 2000, Kim et al., 2000), albeit more reliably at slightly higher doses. Simultaneous 
electrophysiological responses to systemic D1 (full, i.e. not partial) agonism are not known in the mouse literature, although anesthetized data have shown primarily significant decreases in spiking in ventral striatum using iontophoresis (Johansen et al., 1991, Fienberg et al., 1998, Xu et al., 1997, also see Gonon note accompanying Gonon, 1997 online).

Electrophysiological responses in dorsal WT striatum to D1 agonism in vivo may be expected to decrease if partial D1 agonist SKF38393 may be compared to SKF 81297. Systemic administration of SKF 38393 did not differ from SKF 81297 in terms of rescuing elecotrophysiological metrics after DA-depletion (Tseng et al., 2004), and it resulted homogeneously in increases (both in-Isignificant) having no obvious baseline correlation in WT animals. (Xu et al., 2011) (Results from these last two studies are also noteworthy for their opposing results when D1 agonist was given to DA deficient animals; Tseng et al., 2004 found that SKF-81297 decreased spiking, whereas Xu et al., 2011 found a statistically insignificant increase; thus expectations for Pitx3Null dorsal SPNs responses to D1 agonist are less clear.)

Our data showed a genotype difference in baseline correlation with response intensity; stronger specifically in Pitx3Null mice. WT animals showed responses for which we made no planned comparisons for checking against literature expectations, although there was an overall baseline correlation for both genotypes, which suggests a perhaps unexpected result given no previous reports. Nonetheless, Pitx3Null response intensity correlations with baseline spiking were significantly stronger. If WT data control for D1 receptor stimulation enhancing response intensities through a network effect (due possibly to extra-BG D1 receptor stimulation) that favors low-spiking SPNs, then we may interepret the remainder of the genotype difference in this metric to the contribution of super-sensitized D1 receptors.

A similar explanation for genotype's main effect in the 1-DOPA intensity dataset i.e. behavior-relevant WT spiking - may underlie the lack of such an effect in the D1 agonist response intensity dataset; to the extent that WT mice respond behaviorally to SKF 81297, their motor network activity (including SPN spiking) should change accordingly. If this involves any changes in SPN spiking intensity (increasing or decreasing), then as intensities approach those of the Pitx3Null animals, slighter genotype differences become more difficult to detect in such highly variable data. This may indicate the need for a more sensitive metric (or alternatively much more data) in order to show a reduced difference, which is compatible with results from Xu et al., 2011: no effect of DA deficiency in the responses to D1 agonist due to an insignificantly higher spiking increase in paraquat/maneb-treated rats compared to controls.

Indeed, given the intense D1 and D2 receptor expression in SPNs (Levey et al., 1993, Yung et al., 1995) and inherently less motor-inducing effect of D2-SPN stimulation during 1-DOPA (Li \& Zhou, 2013, Bateup et al., 2010, Darmopil et al., 2009), more intense changes in spiking are expected following DA depletion. (Murer \& Moratalla, 2011), but without a way to specify which receptors on which cells will be stimulated and in what network context, assumptions are made instead of controlling for these variables: 
e.g. D1 receptor stimulation having its most intense effect directly at SPNs, especially DA-deficient SPNs, or contributions of receptor stimulation being distinguishable between genotypes on a background of more motor network activity than simply glutamatergic cortical input. Interpreting a receptor contribution to significant SPN spiking changes above and beyond WT responses as a mechanism underlying DAdeficient striatal network activity during behavior will thus require future studies: these should control for changes in intrinsic, afferent, recurrent, and/or reentrant network activity (ideally minimizing SPN spiking variability) so that any aspect of the hyperresponsive spiking in DA deficient SPNs can be isolated to differences in receptor activity.

\section{Striatonigral cell response intensities}

The canonical rate model developed by Albin and Delong (Albin et al, 1989 Wichmann \& Delong, 1996) predict that DA receptor supersensitivity in the DA denervated context would allow DA agonists to lead to electrophysiological (and ultimately behavioral) changes via differential dopamine receptor activation in in-/direct pathways. Specifically D1 receptor signaling on D1-SPNs (which is coupled to G-alphaolf cascades) would be activated to an unusually high degree, which would be expected to exaggerate D1-SPN spike firing simultaneously unusually strong D2 receptor signaling (Gi-coupled) would be expected to decrease the spiking of D2-SPNs. Together, these cellular responseswould yield an overall increase in motor activity, more so in the parkinsonian condition than the usual condition. However, this simplified explanation superficially conflicts to some extents with studies reporting increases in the activity of both D1- and D2-SPNs during movement (Cui et al, 2013) as well as those reporting spiking decreases in vast majorities of SPN recordings during free movement and local application of specific DA agonists (Inase et al., 1997; Kiyatkin and Rebec, 1997 and 1999) and specifically in antidromically identified SPNs during movement induced by systemic DA agonism (Ryan et al, 1989). This could conceivably be due to task-relevant cortical glutamatergic input as a prerequeisite for spiking at all (Mink, 1996, Beeler et al, 2012, Friend \& Kravitz, 2014; also see Discussion).

Antidromically identified cell response intensity differences -polarity being precluded from testing given so few decreases in rate- between genotypes, while not significant in a strict statistical sense, appear to follow rate model expectations and our hypothesis that Pitx3Null SPNs are hyper-responsive in terms of intensity to both 1DOPA and SKF 81297. However, a major problem with this interpretation emerges when considering the possible significance of baseline correlation with response intensity so evident in the other datasets -not tested in this dataset due to such a strong correlation of baseline with genotype and our desire to minimize model complication; Pitx 3 Null baselines are significantly lower than WT, and their responses to both drugs appear to be exaggerated over WT cells. It is thus conceivable that the negative correlation of baselines in other datasets (for SPNs from both genotypes) holds here, which (given baseline differences between genotypes in this dataset) may be responsible for the response intensity difference between genotypes; i.e. this would make it easy to confuse a 
significant effect of baseline on striatonigral response intensities with a significant effect of genotype. Distinguishing this possibility would require more identified cells having higher baselines in Pitx3Null animals and more having lower baselines in WT animals. Yet if the current dataset is representative of the expected ratio of such SPNs, then this experiment appears to be prohibitively difficult since, given our technical capabilities, one to two identified cells per month was the expected rate of acquisition; having two low baseline cells per $8 \mathrm{WT}$ alone would thus require $\sim 5$ times the investment, in order to acquire an $\mathrm{n}$ of even just 4 of these less likely baseline cells (let alone considering animal $\mathrm{N}$ or the fact that all Pitx 3 Null striatonigral cells had low baselines), i.e. a single "rare baseline" identified cell per 3-6 months.

Although inconclusive, these response intensity data still suggest that Pitx3Null DA-deficient striatonigral cells are likely hyper-responsive to D1-receptor stimulation whether that be due to a baseline difference and lor receptor supersensitivity. As with interpreting other datasets, any motor network contribution to the response is still difficult to parse from the direct effect of D1 receptor stimulation. Thus, overall only behaving animal experiments recording from SPNs having identified baseline rates and certain receptor expression as well as isolating DA receptor stimulation as locally in time and space as possible may elucidate differences between lesioned and intact striata of the in vivo pharmacological effects of DA agonism on SPN spiking. A combination of antidromic identification with optogenetic and/or designer receptors activated exclusively by designer drugs (DREADDs) coupled with iontophoretic agonist drug injection during systemic antagonist drug application may be most suitable for this purpose.

\section{Depth differences}

Although depth made a (different) difference to the response polarities in both genotypes, differences in response intensities expected by our hypothesis were notably lacking except in 3-way interactions. Dorsal depth was associated in both genotypes with a more intensely negative correlation of baseline with 1-DOPA response polarities, and the same held true for D1 agonist response polarities in at least Pitx3Null animals (according to planned comparisons). For response intensities, we expected depth to interact with genotype in order to differentiate SPN rate change intensities only in Pitx3Null dorsal from non-dorsal and from all WT; instead it was useful in distinguishing an inverted baseline correlation between genotypes (wherein Pitx3Null non-dorsal SPNs' response intensities were unexpectedly higher) for l-DOPA and in determining that Pitx3Null dorsal baseline correlations were stronger only than WT dorsal baseline correlations in the D1 agonist dataset.

In the case of 1-DOPA, past research indicates that given 1:1 D1:D2-SPNs in the dorsal striatum (Valjent et al., 2009), the original rate model predicts 1:1 increasing:decreasing polarities (and thus no significant group differences) in either genotype, regardless of whether or not they respond to 1-DOPA. Thus, the difference between response polarities dependent on baselines is unexpected by the rate model (since no known results quantify a significant difference between SPN subtypes based on 
purely electrophysiological properties in awake animals [Friend \& Kravitz, 2014], e.g. long-term baseline spiking). This baseline correlation depending additionally on depth is even further unexpected given (again) the lack of results quantifying differences between SPN subtypes in their electrophysiological properties (Friend \& Kravitz, 2014). Either the rate model must be modified to account for an as yet unproven correlation between baseline spiking and SPN subtype, or the model must be updated with another factor having primary influence over spike rates (likely thalamic and cortical glutamatergic input, Mink, 1996, Beeler et al., 2012).

Only 4 of 33 dorsal WT SPNs (from 6 animals) decreased rate responding to a specific D1 receptor agonist; this is exceedingly difficult to reconcile with the rate model's predictions without relying on other factors to explain the phenomenon (e.g. the primacy of cortical input, Beeler et al., 2012, Neve, 2010). Thus, overall polarity results in WT imply - aside from an otherwise paradoxical mechanism through which D1 agonist inhibited spiking in so many non-dorsal SPNs - SPN subtype independence from response polarity. The likelihood that not one of these were D2-SPNs is very low (one sample proportion z-test $p<0.0001$ ), given the knowledge of the $\sim 1: 1$ ratio of SPN subtypes occurring in striata from many species (Valjent et al., 2009). Excluding "insignificant" responses (based on comparing mean rates from 10 or 100 second bins pre- and post-drug) in order to distinguish from the rest of the SPNs those having statistically meaningful increases made the proportion indistinguishable from 50:50; however this approach also uncovered a SPN that significantly decreased mean spiking *(data not shown). This result begs the question of what to expect when stimulating specifically D1 receptors in WT, let alone DA deficient, striatum; thus, the utility of this metric for identifying SPN subtype is dubious and the depth difference considerable enough to require inclusion of other factors predicting rate change polarity.

Of particular note considering depth results from all datasets is that Pitx3Null animals only showed a significant effect of depth in terms of response polarity to D1 agonist, i.e. despite our expectations, depth did not play any role in terms of response intensity differences regardless of drug without comparison to an exaggerated WT depth differential of baseline correlation (1-DOPA) or restricting baseline correlation comparison to dorsal regions between genotypes (D1 agonist). This is born out in (Figures 18-21) where occasional non-dorsal Pitx3Null SPNs have responses apparently as intense as the dorsal SPNs. The supersensitized receptor explanation for such responses would be directly controverted unless these errant SPNs had baselines lower than expected for their response; such cells were not rigorously identified, but the few most obvious had baselines not noticeably lower than their dorsal counterparts (also reflected in the overall results). As explained in the genotype differences section (and more below), results involving the 3-way interaction that included depth differences suggest that altered Pitx3Null motor network functions specific to depth may underlie the effects of DA restoration in the Pitx3Null dorsal striatum compared to WT dorsal (enhancement) and Pitx3Null non-dorsal (comparability) of the negative correlation between SPN baseline and response intensity. 
Overall, aside from the particular relevance of depth in the case of the Pitx 3 Null DA denervation gradient, these results might fit into the literature in terms of taskdependent functional organization of the striatum, provided baseline correlation with response intensity is a natural electrophysiological phenotype of SPN spiking subject to the influence of DA denervation and restoration, which is evident in our data (unplanned comparison in WT dorsal striatum on 1-DOPA). West (1998, also see West et al., 1990) demonstrated more than half (and up to 100\%) of rat dorsolateral striatal (encompassing approximately the dorsal and lateral quadrant of the striatum) SPNs firing sensorimotor related spikes. Graybiel lab studies in primarily dorsolateral (approximately the dorsal and lateral striatal quadrant) striatum depend on such sensorimotor relationships and define their SPN subsets in terms of task-relevance (Kubota et al., 2009, Hernandez et al., 2013, Smith \& Graybiel, 2013); they also showed (Xu et al., 1997) that the D3 receptor mutant mouse's increased locomotor phenotype is likely due to electrophsyiological changes somewhere other than ventral striatum.

Combining insights from both depths, Belin \& Everitt, 2011, showed that chemically inactivating dorsal striatum after a ventral striatal lesion in the opposite hemisphere decreased drug-seeking behavior; they interpreted this effect as an interruption of habit expression. In Pitx3Null mice specifically, Beeler et al., 2008 showed that the dorsal DA lesion precludes a normal locomotor response to cocaine and subsequent sensitization to repeated exposure but that ventral striatal functions like conditioned place preference and cocaine sensitization of sucrose preference were spared. Altogether then, - and to the extent that our non-dorsal data represent ventral striatal function - if indeed Pitx3Null DA denervation prevents the DA-dependent transfer of ventral striatal (goal-directed) SPN spiking to dorsal (stimulus-response habit-related) spiking, then sensorimotor activity in the dorsal Pitx3Null striatum on 1-DOPA or D1 agonist could be expected to reflect something akin to spontaneous formation of motor habits: provided this phenomenon is enacted preferentially through SPNs having lower baselines - possibly striatonigral SPNs -, then this interpretation matches our results showing comparable activity between Pitx3Null depths.

\section{In literature context}

1-DOPA, the most common long term dopamine replacement therapy for PD, relieves the brady- and hypokinesia of parkinsonian patients (i.e. increases self-initiated motor activity) and is eventually associated with motor complications referred to as 1DOPA-induced dyskinesias (Olanow, 2009). These motor effects of acute DA agonism are expected to be mediated by DA receptors located where DA's absence has had the opposite effect of slowing and limiting self-initiated movement. "By what striatal electrophysiological mechanisms does 1-DOPA have a motor stimulating effect?" is the overarching question that we hope to further answer.

More specifically, what effect does 1-DOPA given in the context of DA deficiency have on SPN spiking? SPNs are the largest subpopulation of BG neurons and

are uniquely positioned to integrate cortical input and translate that information to output 
nuclei (Mink, 1996, Beeler et al. 2012, Friend \& Kravitz, 2014); furthermore, they express dopamine receptors heavily (Levey et al 1993; Yung et al 1995), and at least D1SPNs show supersensitized receptors in parkinsonian humans and rats (Corvol et al, 2002; Tong et al, 2004; reviewed in Herve et al, 2011). These receptors are located in the DA-denervated BG (an important network for motor control, Alexander et al., 1991) of PD and of 1-DOPA sensitive animal models, e.g. Pitx3Null mice, 6-OHDA-lesioned rodents. Because the absent nigrostriatal neurons would otherwise innervate only the BG to an appreciable extent, DA from 1-DOPA is regulated as usual in DA sufficient brain areas. Thus DA deficient BG DA receptors are acutely stimulated upon 1-DOPA administration by its neuroactive metabolite, DA, and the subsequent movements must, by definition, be the consequence of a spike output change initiated in any of the subjects' neurons/networks that cause motor activity (in this case the BG motor circuit since all self-initiated animal movement originates in the spiking of such neurons/networks (Nolte, 2002). Taken together, motor activity induced by 1-DOPA is often presumed to precipitate from the action of DA on the spiking of cells in the BG network expressing DA receptors in the context of DA deficiency (such a context including supersensitization, plastic changes from DA loss, dysregulated DA trafficking, etc.). But where is the evidence?

\section{In defense of the causal D1-SPN hypothesis}

D1 SPNS may be the specific cells at which 1-DOPA's effects are necessary for its acute effects on behavior according to the following rationale. The DA that l-DOPA yields stimulates primarily DA receptors, by definition. However, 1-DOPA treatment (i.e. usually combined with a peripheral decarboxylase inhibitor, e.g. benserazide or carbidopa) has shown no detectable behavioral effect in DA-sufficient mice (likely due to their sufficient central DA uptake and metabolism still in place, Abercrombie et al,1990, Kim et al., 2000). $\boldsymbol{l}$-DOPA's motor stimulating effects (both normal and abnormal or dyskinetic) in the chronically DA deficient BG must depend heavily on changes in DA trafficking and/or receptors,i.e. likely to be necessary and sufficient (at the receptor scale) to cause what are known as LID (1-DOPA-induced dyskinesia) in patients, and abnormal involuntary movements in rodents.

The possibility that network plasticity in response to DA denervation could preclude all of these effects is evident when one considers the myriad potential hypotheses formed from the wealth of relevant data: altered DA trafficking (e.g. Lee et al, 2008), compensatory mechanisms (e.g. Iravani et al., 2012), effects on output structures and downstream (e.g. thalamo-cortical changes, simulated or otherwise, Rubin et al, 2012, Hauptmann \& Tass, 2010), all acting on relatively sparsely elucidated mechanisms in the WT striatal context (e.g. D2 receptors filtering low-activity synapses in Bamford et al., 2004, DA agonists affecting all subsets of neurons in all behavioral contexts in Inase et al, 1997, Isomura et al., 2013). Nonetheless, our data allows refinement of the canonical rate model and challenges to it owing to the relative inattention to striatal electrophysiological effects of DA pharmacology in awake animals. 
Based on the evidence reviewed below, it is put forth that D1-SPNs are the likeliest effectors underlying motor activity induced by l-DOPA (i.e. at least a necessary interface between the DA receptors that 1-DOPA stimulates and the behavior that it elicits in the context of chronic DA deficiency). Alternative hypotheses concerning 1-DOPAinduced behavior may exist; only manipulations at the receptor, cell and network levels conducted in as isolated a manner as possible will validate or disprove their differential outcomes with certainty. However, using our current techniques, this is not possible, thus we rely on literature support for this keystone of our rationale, that D1 receptors expressed on D1-SPNs are the primary causative agents in 1-DOPA's behavioral effect in Pitx3Null mice. We support this hypothesis by bolstering the weak electrophysiological data for it (and implicitly for its underlying canonical rate model interpretation). (A similar explanation of D1-SPNs causing AIMs may be found in Murer \& Moratalla, 2011.)

Because of the lack of known extra-BG (i.e. cortical, thalamic, peripheral, etc.) DA receptors to supersensitize or become subject to dysregulated DA trafficking while their DA inputs remain intact, then DA receptors outside of the BG are exceedingly unlikely to underlie this phenomenon by virtue of their otherwise intact sensitivity and homeostatic DA context. All PD models have DA deficiency well-restricted to some portion of the BG. (However, consider Halje et al's, 2012, finding of cortical dopaminergic interruption of 1-DOPA-induced abnormal involuntary movements and less DA terminals in cortex ipsilateral to 6-OHDA lesion as well as Rubin et al's (2012) points about thalamic and cortical DA loss.)

Because 1-DOPA metabolism yields DA, a non-specific DA receptor agonist, both supersensitized D2-family and D1-family receptors may be stimulated to cause the observed motor behaviors. However, much evidence that supports D1- over D2-SPNs playing a necessary role comes from Darmopil et al., 2009, Bateup et al., 2010, Li\&Zhou, 2013 and others:

- Darmopil et al, 2009, used genetic inactivation of D1 or D2 receptors in 6-OHDA lesioned mice in order to show that D1 (and not D2) receptors played a necessary role in 1-DOPA-induced abnormal involuntary movements. It is noteworthy that D1 receptors aren't known to be localized in BG neurons other than D1-SPNs (Kreitzer, 2009; Smith \& Villalba, 2008; Rommelfanger \& Wichmann, 2010) except for a subset of non-bursting subthalamic nucleus (STN) cells, which (as the rest of the STN) more strongly express D5 receptors (the other receptor in the D1 family) and express D5 receptor mRNA much more strongly than that of all other DA receptors;

- Bateup et al, 2010, showed that selective 70\% reduction in D1-SPNs of DARPP32 (a downstream effector of DA receptors) abolished 1-DOPA's motor effect; on the other hand they showed that $\sim 40 \%$ DARPP-32 reduction in D2-SPNs had no effect on 1-DOPA-induced abnormal behaviors but did reduce behavioral effects dependent on D2-family-receptors (e.g. haloperidol-induced catalepsy); 
- Li \& Zhou, 2013, found that increases in horizontal and vertical movements Pitx3Null to D2-family agonist, ropinirole, contrasted considerably with that of 1DOPA whereas D1-family agonist, SKF-81297, resembled 1-DOPA's behavioral response profile much more comparably.

Taken altogether, these studies strongly suggest that D1-SPN spiking is a necessary (and likely sufficient, given the isolation of supersensitized D1 receptors) antecedant at the cell level for acute 1-DOPA-induced behavior.

The evidence discussed in favor of this hypothesis thus far is substantial, however single-unit recordings of SPNs during 1-DOPA-induced abnormal behaviors should correlate the supposedly causal SPN activity to the resulting behaviors on a comparable time scale, lending it more credence, yet precisely these recordings are extremely sparse in both primates (not one as of Boraud et al., 2002, one thereafter Liang et al., 2008) and rodents (Iversen, 2010, pg. 438; Kish et al, 1999, Chen et al, 2001, Halje et al, 2012.) Anesthetized animals have been studied in much more detail and their SPNs for the most part show responses similar to those in awake animals but having drastically different firing rates than during waking (Kish et al, 1999) and are therefore of questionable fidelity to waking circumstances, especially in the sensorimotor-related dorsolateral striatum (West, 1998.)

Pitx3Null mice are an appropriate model of the motor activity induced by 1-DOPA for many reasons. Their l-DOPA-induced motor activity (van den Munckoff et al, 2006), which is absent in WT animals (Ding et al, 2007), is responsive to anti-LID therapies, amantadine and buspirone (Francardo and Cenci, 2014), and molecular markers of DA receptor supersensitivity similar to 1-DOPA-induced dyskinesia models arise in the Pitx3Null striatum subsequent to 1-DOPA treatment (Francardo \& Cenci, 2014). Together, these indicate interactions between the conceptual levels of behavior and receptors similar to patients and other animal models concerning the l-DOPA sensitivity that arises after DA deficiency. Finally these mice avoid the pitfalls of toxin-lesioned models (e.g. reproducibility of lesion, dependability of response to 1-DOPA across animals; Li \& Zhou, 2013).

Clearly one problem with using an SPN's change in spiking to characterize receptor expression (and thus projection target as well as putative network contribution) is the ambiguity of responses to DA. Whereas D1-SPNs are expected in the standard model to increase rate in response to stimulation of D1 receptors according to their downstream effectors, and D2-SPNs should show the opposite effect due to D2 receptor stimulation, the ratio of responses seen in this study and others (e.g. Ruskin et al., 1999) imply that further methods are required in order to distinguish D1- from D2- SPNs in vivo, let alone to distinguish the opposite responses that further parse each of those subpopulations since their pharmacological responses could be so similar (e.g. given our results or those of others like Inase et al., 1997, Claussen \& Dafny, 2015). 
Ryan et al. 1989 tried to disambiguate responses to amphetamine in healthy, mobile rats by antidromically activating putative D1-SPNs (as we have done here for the Pitx3 mutant mouse), but many of the identified cells responded unexpectedly; they showed general decreases in firing over tens of minutes and specific decreases using behavioral periods matched pre- and post-drug exposure (akin to a "behavioral clamp" experiment, e.g. spiking considered only during head bobbing, only during sniffing, etc.), although some did increase. Rebec, 1998, tried iontophoretic application of DA simultaneously with glutamate into the striatum of unrestrained rats in order to provide more support for the hypothesis that motor-related SPNs were responding to drug (amphetamine in his previous studies), and not to other motor-related brain activity, cortical input or sensory feedback among others (for some of which he performed separate studies to rule out their possibilities).

In awake rats, Kish et al, 1999, observed that SPN spiking increased on average after apomorphine (a mixed D1/D2 agonist) in 6-OHDA-lesioned striata whereas apomorphine was without effect on the respective unlesioned striata and unilateral saline control animal striata. They concluded that firing rate sensitivity to DA was increased in DA-deficient SPNs. Chen et al, 2001, saw the same dose of apomorphine, in contrast, decrease rates in lesioned striata on average (whose baseline rates were already increased from control). They concluded that DA's function was inhibitory to SPNs and may thereby affect action selection. However, neither study distinguished cells having different rate response polarities; thus, by conflating rate increases with decreases when averaging all rate changes, mechanisms more refined than homogenous rate coding across cells (known to be of different subtypes) were precluded from commentary. Accordingly, Halje et al, 2012, reported that 1-DOPA injection after unilateral 6-OHDA lesion indeed leads to both increases and decreases in SPN spiking, but conclusions were focused entirely on an oscillatory cortical activity contributing to dyskinesia. Each of these studies left SPNs unidentified, which weakened their explanatory power, thus limiting clinically relevant interpretations (especially those possible within the canonical framework).

The resulting dichotomy of responses to D1 agonist herein (in-/decreases exemplified best in Figures 18-21) was therefore not unexpected (also see other studies classifying SPNs according primarily to task-relevance before or in addition to DA response, e.g. Hernandez et al, 2013, Inase et al., 1997). However, promisingly, SPNs in Pitx3Null animals, both increasing and decreasing, responded more intensely accompanying motor activity induced by 1-DOPA.

Inasmuch as our conclusions are similar, our results are in agreement with Kish et al. 1999, in the rat 6-OHDA model of LID. We also extend their results in the following ways:

- by the presence of a different style of induced abnormal behavior -"three-paw dyskinesia" in Pitx3Null (Ding et al, 2007) instead of contralateral rotation-due to the different DA lesion in Pitx3Null mice (Li et al, 2015); 
- by antidromically identifying putative D1-SPNs as being particularly and significantly affected by DA depletion and likely by DA agonism in that context.

To this last point, although there is no compelling evidence from awake, behaving animals that specific activation of D1 receptors causes strictly, or even primarily, increases in D1-SPN spiking (as opposed to more complex, modest, or other unexpected effects on SPN spiking), such responses are expected in at least some subpopulation of D1-SPNs secondary to D1 receptor stimulation. This is especially so given concurrent supersensitization of DA receptor signaling after DA depletion, all based on the prediction of the canonical rate model (also supported via parkinsonian primate BG cell responses to DA agonists, Boraud et al, 2002, and many rodent studies, Ruskin et al, 1999, Waszczak, 1984, Iversen, 2010, pg 438) and some in vitro rodent PD model data (Plotkin et al, 2011). From a purely electriophysiological persepctive, because optical stimulation of D1-SPNs is known to activate behavior, they would be expected to increase firing based on their being positioned conceptually at the scale between DAdenervated, supersensitized DA receptors and behavior induced by l-DOPA in the putative causal pathway outlined above.

Two WT identified SPNs (not shown since they were below the threshold for being considered dorsal) decreased activity after D1 agonism; these do not fit the canonical rate model but warrant comment since they have been seen before. They may be disfacilitated (Mahon et al, 2006) by a behavior-related, silencing of glutamatergic inputs originating in the cortex and thalamus, discussed more below. Alternatively, in the case of 1-DOPA injections D2 receptor agonism may silence these SPNs (Cepeda \& Levine, 1998; Plotkin et al, 2011). Although supported in non-waking animal studies and in vitro, these interpretations remain speculative.

Rate-decreasing SPNs identified as D1 expressing (e.g. Fig. 7, Ryan et al, 1989) are difficult to account for because their spike activity in relation to motor behavior is exactly opposite of that expected based on the canonical rate model; thus this subpopulation warrants special attention. Its existence alone is sufficient evidence to warrant reconsidering the model, which has been easily resolved by adding one more level of complexity: that of behaviorally relevant glutamatergic inputs. Behavioral relevance may be built into the topographical nature of the corticostriatal projections: a principled simulation by Beeler et al, 2012, endowed these projections with the ability to facilitate SPN spiking in a task-dependent way. Thus relevant spiking in the cortex was necessary before DA could exert its effect, and the model accounted for DA effects they observed in vivo. In other words, one possible explanation for the motor-relatedness of any single SPN presupposes glutamatergic input sufficient to cause SPN spiking before DA may be allowed to have its canonical rate effect on spiking.

In vitro, other unpublished results from our lab indicate that dorsal Pitx3Null D1SPNs are more sensitive to D1 agonism in terms of excitability and current pulse-induced firing. Observing these results in a preparation lacking much cortical and thalamic glutamatergic input is even more impressive considering that such input has been shown to be necessary for not only SPN spiking (Surmeier et al. 2007) but the D1 agonist effect 
as well (Plotkin et al, 2011). Considering this point in conjunction with the findings that glutamatergic inputs are the primary source of glutamatergic excitation to SPNs (Surmeier et al. 2007), then it stands to reason that specific cortical/thalamic activity gives way to specific striatal activity in a topographic manner and that this topography may underlie the specialized functions of specific locales of striatum (Beeler et al, 2012).

\section{Discussion of Striatal Fibers of Passage Being Mistaken for FSIs}

Given the width of these waveforms compared to SPNs, and their rates, they would most likely be considered FSIs (PV+ GABAergic interneurons). Even a more detailed look at their patterns during wake and sleep might support that conclusion. However, while the degree of evidence supporting each cell's identity differs, their matching characteristics nonetheless bolster the hypothesis that they are neither interneurons nor SPNs.

The results shown are very unlikely to be orthodromic spikes from the midbrain since collisions would extremely unexpected by any known mechanism in that case; the consistency of a precise temporal sequence of stimulus then response, the collisions, and the refractory period all point to these responses as axonal spike propagation.

Myelinated cortical axons exhibit conduction velocities on the order of high singles to tens of meters per second in cats (Jinnai \& Matsuda, 1979) and macaques (Turner \& Delong, 2000). Sensorimotor cortical axons course more densely in the dorsolateral striatum. (Wilson, 1987, Kubota et al, 2009, Schwarz \& Chakrabarti, 2015) Given the only known major projections to striatum that would approach the stimulating electrode placed in this study, these fibers would presumably be of cortical origin. In summary, these characteristics (widespread instances of bundled cell processes projecting through the striatum to or beyond midbrain and having conduction velocities in the single meters per second) increase the candidacy of the cortex as the origin of these fibers.

\section{Conclusions}

Overall, we have bolstered heretofore weak electrophysiological data supporting the hypothesis (modeled on the in/direct pathways concept) that hyper-responsive SPNs mediate motor activity initiated via DA-deficient SPN DA receptor signaling by showing clear distinction in SPN spiking changes between dorsal WT and DA-denervated Pitx3Null striatum in response to DA agonists. Furthermore, where DA denervation is chronically $<1 \%$ that of WT (Li\&Zhou, 2013), baseline striatonigral spiking is much less than where there is full DA innervation in the complementary WT dorsal striatum. Fitting our results into the context of previously published literature concerning the causes of motor activity induced by 1-DOPA, we posit that lowering 1-DOPA dose reduces dyskinesias but at the cost of some efficacy in restoring motor activity to the otherwise parkinsonian state. This may imply that 1-DOPA's induction of both normal and 
dyskinetic motor activity may be inextricably linked to a common pharmacological mechanism involving DA signaling in the BG.

Furthermore, while the given evidence for fibers of passage being mistaken as striatal FSIs does not irrefutably prove the identity/somatic location of the units, it nonetheless casts strong doubt on the validity of the assumption that all striatal units having narrow waveforms and high rate are interneurons. Previous findings may thus be reconsidered, and future reports more cautiously interpreted in order to potentially resolve apparently contradictory results. This may also represent an opportunity to improve the utility of striatal recordings; e.g., upon improving this technique's reliability and identifying these fibers, one may be able to improve the corroboration of striatal data with somewhat coextensive extrastriatal projection cells in real-time without requiring extra electrodes. Future experiments should attempt to distinguish in vivo identification criteria between FSIs and fibers of passage through the striatum, most likely cortical. 


\section{LIST OF REFERENCES}

Abercrombie, E. D., Bonatz, A. E., \& Zigmond, M. J. (1990). Effects ofl-DOPA on extracellular dopamine in striatum of normal and 6-hydroxydopamine-treated rats. Brain Research, 525(1), 36-44. https://doi.org/10.1016/0006-8993(90)91318-B

Albin, R. L., Young, A. B., \& Penney, J. B. (1989). The functional anatomy of basal ganglia disorders. Trends in Neurosciences, 12(10), 366-375.

https://doi.org/10.1016/0166-2236(89)90074-X

Alexander, G. E., Crutcher, M. D., \& DeLong, M. R. (1991). Chapter 6 Basal gangliathalamocortical circuits: Parallel substrates for motor, oculomotor, "prefrontal" and "limbic" functions. In Progress in Brain Research (Vol. 85, pp. 119-146). Elsevier. https://doi.org/10.1016/S0079-6123(08)62678-3

Bamford, N. S., Zhang, H., Schmitz, Y., Wu, N.-P., Cepeda, C., Levine, M. S., ... Sulzer, D. (2004). Heterosynaptic dopamine neurotransmission selects sets of corticostriatal terminals. Neuron, 42(4), 653-663.

Ballion, B., Frenois, F., Zold, C. L., Chetrit, J., Murer, M. G., \& Gonon, F. (2009). D2 receptor stimulation, but not $\mathrm{D} 1$, restores striatal equilibrium in a rat model of Parkinsonism. Neurobiology of Disease, 35(3), 376-384.

https://doi.org/10.1016/j.nbd.2009.05.019

Barnes, Terra D., Yasuo Kubota, Dan Hu, Dezhe Z. Jin, and Ann M. Graybiel. 2005. "Activity of Striatal Neurons Reflects Dynamic Encoding and Recoding of Procedural Memories." Nature 437 (7062): 1158-61. https://doi.org/10.1038/nature04053.

Bastide, M. F., Meissner, W. G., Picconi, B., Fasano, S., Fernagut, P.-O., Feyder, M., ... Bézard, E. (2015). Pathophysiology of L-dopa-induced motor and non-motor complications in Parkinson's disease. Progress in Neurobiology, 132, 96-168. https://doi.org/10.1016/j.pneurobio.2015.07.002

Bateup, H. S., Santini, E., Shen, W., Birnbaum, S., Valjent, E., Surmeier, D. J., ... Greengard, P. (2010). Distinct subclasses of medium spiny neurons differentially regulate striatal motor behaviors. Proceedings of the National Academy of Sciences, 107(33), 14845-14850. https://doi.org/10.1073/pnas.1009874107

Beeler, J. A., Cao, Z. F. H., Kheirbek, M. A., \& Zhuang, X. (2008). Loss of cocaine locomotor response in Pitx3-deficient mice lacking a nigrostriatal pathway. Neuropsychopharmacology, 34(5), 1149-1161.

Beeler, J. A., Cao, Z. F. H., Kheirbek, M. A., Ding, Y., Koranda, J., Murakami, M., ... Zhuang, X. (2009). Dopamine-dependent motor learning: Insight into levodopa's longduration response. Annals of Neurology, 67(5), 639-647. 
Beeler, J. A., Frank, M. J., McDaid, J., Alexander, E., Turkson, S., Sol Bernandez, M., ... Zhuang, X. (2012). A Role for Dopamine-Mediated Learning in the Pathophysiology and Treatment of Parkinson's Disease. Cell Reports. Retrieved from http://www.sciencedirect.com/science/article/pii/S2211124712004111

Belin, D., \& Everitt, B. J. (2008). Cocaine seeking habits depend upon dopaminedependent serial connectivity linking the ventral with the dorsal striatum. Neuron, 57(3), $432-441$.

Berke, J. D., M. Okatan, J. Skurski, and H. B. Eichenbaum. 2004. "Oscillatory Entrainment of Striatal Neurons in Freely Moving Rats.” Neuron 43 (6): 883-896.

Berke, J. D. 2008. "Uncoordinated Firing Rate Changes of Striatal Fast-Spiking Interneurons during Behavioral Task Performance." Journal of Neuroscience 28 (40): 10075-80. https://doi.org/10.1523/JNEUROSCI.2192-08.2008.

Berke, Joshua D. 2011. "Functional Properties of Striatal Fast-Spiking Interneurons." Frontiers in Systems Neuroscience 5. https://doi.org/10.3389/fnsys.2011.00045.

Boraud, T., Bezard, E., Bioulac, B., \& Gross, C. E. (2002). From single extracellular unit recording in experimental and human Parkinsonism to the development of a functional concept of the role played by the basal ganglia in motor control. Progress in Neurobiology, 66(4), 265-283. https://doi.org/10.1016/S0301-0082(01)00033-8

Cenci, M. A., \& Konradi, C. (2010). Maladaptive striatal plasticity in 1-DOPA-induced dyskinesia. In Progress in Brain Research (Vol. 183, pp. 209-233). Elsevier. Retrieved from http://linkinghub.elsevier.com/retrieve/pii/S0079612310830110

Cepeda, C., \& Levine, M. S. (1998). Dopamine and N-methyl-D-aspartate receptor interactions in the neostriatum. Developmental Neuroscience, 20(1), 1-18.

Chen, M. T., Morales, M., Woodward, D. J., Hoffer, B. J., \& Janak, P. H. (2001). In Vivo Extracellular Recording of Striatal Neurons in the Awake Rat Following Unilateral 6Hydroxydopamine Lesions. Experimental Neurology, 171(1), 72-83.

Claussen, C. M., \& Dafny, N. (2015). Caudate neuronal recording in freely behaving animals following acute and chronic dose response methylphenidate exposure.

Pharmacology Biochemistry and Behavior, 136, 21-30.

https://doi.org/10.1016/j.pbb.2015.06.003

Corvol, J.-C. (2004). Persistent Increase in Olfactory Type G-Protein Subunit Levels May Underlie D1 Receptor Functional Hypersensitivity in Parkinson Disease. Journal of Neuroscience, 24(31), 7007-7014. https://doi.org/10.1523/JNEUROSCI.0676-04.2004 
Costa, R. M., Lin, S.-C., Sotnikova, T. D., Cyr, M., Gainetdinov, R. R., Caron, M. G., \& Nicolelis, M. A. L. (2006). Rapid Alterations in Corticostriatal Ensemble Coordination during Acute Dopamine-Dependent Motor Dysfunction. Neuron, 52(2), 359-369. https://doi.org/10.1016/j.neuron.2006.07.030

Cui, G., Jun, S. B., Jin, X., Pham, M. D., Vogel, S. S., Lovinger, D. M., \& Costa, R. M. (2013). Concurrent activation of striatal direct and indirect pathways during action initiation. Nature. Retrieved from http://www.nature.com/nature/journal/vaop/ncurrent/full/nature11846.html

Darmopil, S., Martín, A. B., De Diego, I. R., Ares, S., \& Moratalla, R. (2009). Genetic inactivation of dopamine D1 but not D2 receptors inhibits L-DOPA-induced dyskinesia and histone activation. Biol. Psychiatry, 66, 603-613.

Delfino, M., Kalisch, R., Czisch, M., Larramendy, C., Ricatti, J., Taravini, I. R., ... Gershanik, O. S. (2007). Mapping the effects of three dopamine agonists with different dyskinetogenic potential and receptor selectivity using pharmacological functional magnetic resonance imaging. Neuropsychopharmacology, 32(9), 1911-1921.

Delis, F., Mitsacos, A., \& Giompres, P. (2013). Lesion of the cerebellar paravermis increases dopamine D1 receptor levels in the contralateral striatum. Journal of Chemical Neuroanatomy, 47, 35-41. https://doi.org/10.1016/j.jchemneu.2012.10.004

Ding, Y., Restrepo, J., Won, L., Hwang, D.-Y., Kim, K.-S., \& Kang, U. J. (2007). Chronic 3,4-dihydroxyphenylalanine treatment induces dyskinesia in aphakia mice, a novel genetic model of Parkinson's disease. Neurobiology of Disease, 27(1), 11-23. https://doi.org/10.1016/j.nbd.2007.03.013

Ferguson, J. E., C. Boldt, and A. D. Redish. 2009. "Creating Low-Impedance Tetrodes by Electroplating with Additives." Sensors and Actuators A: Physical 156 (2): 388-393.

Feyder, M., Bonito-Oliva, A., \& Fisone, G. (2011). L-DOPA-Induced Dyskinesia and Abnormal Signaling in Striatal Medium Spiny Neurons: Focus on Dopamine D1 Receptor-Mediated Transmission. Frontiers in Behavioral Neuroscience, 5. https://doi.org/10.3389/fnbeh.2011.00071

Fienberg, A. A. (1998). DARPP-32: Regulator of the Efficacy of Dopaminergic Neurotransmission. Science, 281(5378), 838-842. https://doi.org/10.1126/science.281.5378.838

Fleming, S. M., Ekhator, O. R., \& Ghisays, V. (2013). Assessment of Sensorimotor Function in Mouse Models of Parkinson's Disease. Journal of Visualized Experiments, (76). https://doi.org/10.3791/50303 
Francardo, V., \& Cenci, M. A. (2014). Investigating the molecular mechanisms of LDOPA-induced dyskinesia in the mouse. Parkinsonism \& Related Disorders, 20 Suppl 1, S20-22. https://doi.org/10.1016/S1353-8020(13)70008-7

Franco, V., \& Turner, R. S. (2012). Testing the contributions of striatal dopamine loss to the genesis of parkinsonian signs. Neurobiology of Disease, 47(1), 114-125. https://doi.org/10.1016/j.nbd.2012.03.028

Freeze, B. S., Kravitz, A. V., Hammack, N., Berke, J. D., \& Kreitzer, A. C. (2013). Control of Basal Ganglia Output by Direct and Indirect Pathway Projection Neurons. Journal of Neuroscience, 33(47), 18531-18539.

https://doi.org/10.1523/JNEUROSCI.1278-13.2013

Friend, D. M., \& Kravitz, A. V. (2014). Working together: basal ganglia pathways in action selection. Trends in Neurosciences, 37(6), 301-303.

https://doi.org/10.1016/j.tins.2014.04.004

Gage, Gregory J., Colin R. Stoetzner, Alexander B. Wiltschko, and Joshua D. Berke. 2010. "Selective Activation of Striatal Fast-Spiking Interneurons during Choice Execution.” Neuron 67 (3): 466-79. https://doi.org/10.1016/j.neuron.2010.06.034.

Gerfen, C. R., Miyachi, S., Paletzki, R., \& Brown, P. (2002). D1 dopamine receptor supersensitivity in the dopamine-depleted striatum results from a switch in the regulation of ERK1/2/MAP kinase. The Journal of Neuroscience, 22(12), 5042-5054.

Gittis, A. H., D. K. Leventhal, B. A. Fensterheim, J. R. Pettibone, J. D. Berke, and A. C. Kreitzer. 2011. "Selective Inhibition of Striatal Fast-Spiking Interneurons Causes Dyskinesias." Journal of Neuroscience 31 (44): 15727-31. https://doi.org/10.1523/JNEUROSCI.3875-11.2011.

Gonon, F. (1997). Prolonged and extrasynaptic excitatory action of dopamine mediated by D1 receptors in the rat striatum in vivo. The Journal of Neuroscience, 17(15), 59725978.

Graveland, G. A., and M. DiFiglia. 1985. "The Frequency and Distribution of MediumSized Neurons with Indented Nuclei in the Primate and Rodent Neostriatum." Brain Research 327 (1-2): 307-11.

Haber, S. N., Fudge, J. L., \& McFarland, N. R. (2000). Striatonigrostriatal pathways in primates form an ascending spiral from the shell to the dorsolateral striatum. The Journal of Neuroscience: The Official Journal of the Society for Neuroscience, 20(6), 2369-2382.

Halje, P., Tamte, M., Richter, U., Mohammed, M., Cenci, M. A., \& Petersson, P. (2012). Levodopa-Induced Dyskinesia Is Strongly Associated with Resonant Cortical Oscillations. Journal of Neuroscience, 32(47), 16541-16551. https://doi.org/10.1523/JNEUROSCI.3047-12.2012 
Hauptmann, C., \& Tass, P. A. (2010). Restoration of segregated, physiological neuronal connectivity by desynchronizing stimulation. Journal of Neural Engineering, 7(5), 056008. https://doi.org/10.1088/1741-2560/7/5/056008

Heinricher, Mary M. "Principles of Extracellular Single-Unit Recording." In: Israel, Zvi, and Kim J. Burchiel, eds. 2004. Microelectrode Recording in Movement Disorder Surgery. Stuttgart: Georg Thieme Verlag. https://doi.org/10.1055/b-002-56126.

Hernandez, L. F., Y. Kubota, D. Hu, M. W. Howe, N. Lemaire, and A. M. Graybiel. 2013. "Selective Effects of Dopamine Depletion and L-DOPA Therapy on LearningRelated Firing Dynamics of Striatal Neurons." Journal of Neuroscience 33 (11): 4782 95. https://doi.org/10.1523/JNEUROSCI.3746-12.2013.

Hervé, D. (2011). Identification of a Specific Assembly of the G Protein Golf as a Critical and Regulated Module of Dopamine and Adenosine-Activated cAMP Pathways in the Striatum. Frontiers in Neuroanatomy, 5. https://doi.org/10.3389/fnana.2011.00048

Hornykiewicz, O. (2001). Chemical neuroanatomy of the basal ganglia-normal and in Parkinson's disease. Journal of Chemical Neuroanatomy, 22(1), 3-12.

Huot, P. (2015). L-DOPA-induced dyskinesia, is striatal dopamine depletion a requisite? Journal of the Neurological Sciences, 351(1-2), 9-12.

https://doi.org/10.1016/j.jns.2015.02.041

Hwang, D. Y., Ardayfio, P., Kang, U. J., Semina, E. V., \& Kim, K. S. (2003). Selective loss of dopaminergic neurons in the substantia nigra of Pitx3-deficient aphakia mice. Molecular Brain Research, 114(2), 123-131.

Hwang, D.-Y. (2005). 3,4-Dihydroxyphenylalanine Reverses the Motor Deficits in Pitx3Deficient Aphakia Mice: Behavioral Characterization of a Novel Genetic Model of Parkinson's Disease. Journal of Neuroscience, 25(8), 2132-2137. https://doi.org/10.1523/JNEUROSCI.3718-04.2005

Inase, M., Li, B.-M., \& Tanji, J. (1997). Dopaminergic modulation of neuronal activity in the monkey putamen through D1 and D2 receptors during a delayed Go/Nogo task. Experimental Brain Research, 117(2), 207-218.

Iravani, M. M., McCreary, A. C., \& Jenner, P. (2012). Striatal plasticity in Parkinson's disease and L-DOPA induced dyskinesia. Parkinsonism \& Related Disorders, 18, S123S125. https://doi.org/10.1016/S1353-8020(11)70038-4

Isomura, Y., Takekawa, T., Harukuni, R., Handa, T., Aizawa, H., Takada, M., \& Fukai, T. (2013). Reward-Modulated Motor Information in Identified Striatum Neurons. Journal of Neuroscience, 33(25), 10209-10220. https://doi.org/10.1523/JNEUROSCI.0381$\underline{13.2013}$ 
Iversen, L. (2010). Dopamine handbook. Oxford ; New York: Oxford University Press.

Jacobs, F. M. J., Smits, S. M., Noorlander, C. W., von Oerthel, L., van der Linden, A. J. A., Burbach, J. P. H., \& Smidt, M. P. (2007). Retinoic acid counteracts developmental defects in the substantia nigra caused by Pitx3 deficiency. Development, 134(14), 26732684. https://doi.org/10.1242/dev.02865

Jinnai, K., and Y. Matsuda. 1979. "Neurons of the Motor Cortex Projecting Commonly on the Caudate Nucleus and the Lower Brain Stem in the Cat." Neuroscience Letters 13 (2): 121-26.

Joel, D., \& Weiner, I. (1994). The organization of the basal ganglia-thalamocortical circuits: Open interconnected rather than closed segregated. Neuroscience, 63(2), 363379. https://doi.org/10.1016/0306-4522(94)90536-3

Johansen, P. A., Hu, X.-T., \& White, F. J. (1991). Relationship between D 1 dopamine receptors, adenylate cyclase, and the electrophysiological responses of rat nucleus accumbens neurons. Journal of Neural Transmission / General Section JNT, 86(2), 97113. https://doi.org/10.1007/BF01250571

Kas, M. J. H., van der Linden, A. J. A., Oppelaar, H., von Oerthel, L., Ramakers, G. M. J., \& Smidt, M. P. (2008). Phenotypic segregation of aphakia and Pitx3-null mutants reveals that Pitx 3 deficiency increases consolidation of specific movement components. Behavioural Brain Research, 186(2), 208-214. https://doi.org/10.1016/j.bbr.2007.08.032

Kim, D. S., Szczypka, M. S., \& Palmiter, R. D. (2000). Dopamine-deficient mice are hypersensitive to dopamine receptor agonists. The Journal of Neuroscience: The Official Journal of the Society for Neuroscience, 20(12), 4405-4413.

Kish, L. J., Palmer, M. R., \& Gerhardt, G. A. (1999). Multiple single-unit recordings in the striatum of freely moving animals: effects of apomorphine and D-amphetamine in normal and unilateral 6-hydroxydopamine-lesioned rats. Brain Research, 833(1), 58-70.

Kita, H., \& Kita, T. (2011a). Cortical Stimulation Evokes Abnormal Responses in the Dopamine-Depleted Rat Basal Ganglia. Journal of Neuroscience, 31(28), 10311-10322. https://doi.org/10.1523/JNEUROSCI.0915-11.2011

Kita, H., \& Kita, T. (2011b). Role of striatum in the pause and burst generation in the globus pallidus of 6-OHDA-treated rats. Frontiers in Systems Neuroscience, 5. Retrieved from http://www.ncbi.nlm.nih.gov/pmc/articles/PMC3113166/

Kiyatkin, E. A., \& Rebec, G. V. (1997). Iontophoresis of amphetamine in the neostriatum and nucleus accumbens of awake, unrestrained rats. Brain Research, 771(1), 14-24.

https://doi.org/10.1016/S0006-8993(97)00689-6 
Kiyatkin, E. A., \& Rebec, G. V. (1999). Striatal neuronal activity and responsiveness to dopamine and glutamate after selective blockade of D1 and D2 dopamine receptors in freely moving rats. The Journal of Neuroscience, 19(9), 3594-3609.

Kravitz, A. V., Freeze, B. S., Parker, P. R. L., Kay, K., Thwin, M. T., Deisseroth, K., \& Kreitzer, A. C. (2010). Regulation of parkinsonian motor behaviours by optogenetic control of basal ganglia circuitry. Nature, 466(7306), 622-626.

Kreitzer, A. C. (2009). Physiology and Pharmacology of Striatal Neurons. Annual Review of Neuroscience, 32(1), 127-147. https://doi.org/10.1146/annurev.neuro.051508.135422

Kubota, Y., J. Liu, D. Hu, W. E. DeCoteau, U. T. Eden, A. C. Smith, and A. M. Graybiel. 2009. "Stable Encoding of Task Structure Coexists With Flexible Coding of Task Events in Sensorimotor Striatum." Journal of Neurophysiology 102 (4): 2142-60. https://doi.org/10.1152/jn.00522.2009.

Lee, J., Zhu, W.-M., Stanic, D., Finkelstein, D. I., Horne, M. H., Henderson, J., ... Horne, M. K. (2008). Sprouting of dopamine terminals and altered dopamine release and uptake in Parkinsonian dyskinaesia. Brain, 131(6), 1574-1587. https://doi.org/10.1093/brain/awn085

Lee, Kwang, Sandra M. Holley, Justin L. Shobe, Natalie C. Chong, Carlos Cepeda, Michael S. Levine, and Sotiris C. Masmanidis. 2017. "Parvalbumin Interneurons Modulate Striatal Output and Enhance Performance during Associative Learning." Neuron 93 (6): 1451-1463.e4. https://doi.org/10.1016/j.neuron.2017.02.033.

Levey, A. I., Hersch, S. M., Rye, D. B., Sunahara, R. K., Niznik, H. B., Kitt, C. A., ... Ciliax, B. J. (1993). Localization of D1 and D2 dopamine receptors in brain with subtype-specific antibodies. Proceedings of the National Academy of Sciences, 90(19), $8861-8865$.

Li, L., \& Zhou, F.-M. (2013). Parallel dopamine D1 receptor activity dependence of 1Dopa-induced normal movement and dyskinesia in mice. Neuroscience, 236, 66-76. https://doi.org/10.1016/j.neuroscience.2012.12.065

Li, L., Sagot, B., \& Zhou, F.-M. (2015). Similar 1 -dopa-stimulated motor activity in mice with adult-onset 6-hydroxydopamine-induced symmetric dopamine denervation and in transcription factor Pitx 3 null mice with perinatal-onset symmetric dopamine denervation. Brain Research, 1615, 12-21. https://doi.org/10.1016/j.brainres.2015.04.011

Liang, L., DeLong, M. R., \& Papa, S. M. (2008). Inversion of Dopamine Responses in Striatal Medium Spiny Neurons and Involuntary Movements. Journal of Neuroscience, 28(30), 7537-7547. https://doi.org/10.1523/JNEUROSCI.1176-08.2008 
Liao, Y. F., Tsai, M. L., Yen, C. T., \& Cheng, C. H. (2011). A simple method for fabricating microwire tetrode with sufficient rigidity and integrity without a heat-fusing process. Journal of Neuroscience Methods, 195(2), 211-215.

Luk, K.C, and A.F Sadikot. 2001. "GABA Promotes Survival but Not Proliferation of Parvalbumin-Immunoreactive Interneurons in Rodent Neostriatum: An in Vivo Study with Stereology." Neuroscience 104 (1): 93-103. https://doi.org/10.1016/S03064522(01)00038-0.

Mahon, S., Vautrelle, N., Pezard, L., Slaght, S. J., Deniau, J.-M., Chouvet, G., \& Charpier, S. (2006). Distinct Patterns of Striatal Medium Spiny Neuron Activity during the Natural Sleep-Wake Cycle. Journal of Neuroscience, 26(48), 12587-12595. https://doi.org/10.1523/JNEUROSCI.3987-06.2006

Mallet, N. 2005. "Feedforward Inhibition of Projection Neurons by Fast-Spiking GABA Interneurons in the Rat Striatum In Vivo." Journal of Neuroscience 25 (15): 3857-69. https://doi.org/10.1523/JNEUROSCI.5027-04.2005.

Mallet, N. 2006. "Cortical Inputs and GABA Interneurons Imbalance Projection Neurons in the Striatum of Parkinsonian Rats." Journal of Neuroscience 26 (14): 3875-84. https://doi.org/10.1523/JNEUROSCI.4439-05.2006.

Mink, J. W. (1996). The basal ganglia: focused selection and inhibition of competing motor programs. Progress in Neurobiology, 50(4), 381-425.

Murer, M. G., \& Moratalla, R. (2011). Striatal Signaling in L-DOPA-Induced Dyskinesia: Common Mechanisms with Drug Abuse and Long Term Memory Involving D1 Dopamine Receptor Stimulation. Frontiers in Neuroanatomy, 5. https://doi.org/10.3389/fnana.2011.00051

Nambu, A. (2008). Seven problems on the basal ganglia. Current Opinion in Neurobiology, 18(6), 595-604.

Neve, K. (2009). The Dopamine Receptors. Springer Science \& Business Media.

Nisenbaum, E. S., Grace, A. A., \& Berger, T. W. (1992). Functionally distinct subpopulations of striatal neurons are differentially regulated by gabaergic and dopaminergic inputs?II. In vitro analysis. Neuroscience, 48(3), 579-593. https://doi.org/10.1016/0306-4522(92)90403-O

Nolte, J. (2002). The human brain: an introduction to its functional anatomy (5th ed). St. Louis, Mo: Mosby.

O’Hare, Justin K, Haofang Li, Namsoo Kim, Erin Gaidis, Kristen Ade, Jeff Beck, Henry Yin, and Nicole Calakos. 2017. "Striatal Fast-Spiking Interneurons Selectively Modulate 
Circuit Output and Are Required for Habitual Behavior.” ELife 6 (September). https://doi.org/10.7554/eLife.26231.

Olanow, C. W., Stern, M. B., \& Sethi, K. (2009). The scientific and clinical basis for the treatment of Parkinson disease (2009). Neurology, 72(21 Suppl 4), S1-136. https://doi.org/10.1212/WNL.0b013e3181a1d44c

Pavón, N., Martín, A. B., Mendialdua, A., \& Moratalla, R. (2006). ERK Phosphorylation and FosB Expression Are Associated with L-DOPA-Induced Dyskinesia in Hemiparkinsonian Mice. Biological Psychiatry, 59(1), 64-74. https://doi.org/10.1016/j.biopsych.2005.05.044

Picconi, B., Centonze, D., Rossi, S., Bernardi, G., \& Calabresi, P. (2004). Therapeutic doses of L-dopa reverse hypersensitivity of corticostriatal D2-dopamine receptors and glutamatergic overactivity in experimental parkinsonism. Brain, 127(7), 1661-1669. https://doi.org/10.1093/brain/awh190

Plotkin, J. L., Day, M., \& Surmeier, D. J. (2011). Synaptically driven state transitions in distal dendrites of striatal spiny neurons. Nature Neuroscience, 14(7), 881-888. https://doi.org/10.1038/nn.2848

Prieto, G. A., Perez-Burgos, A., Fiordelisio, T., Salgado, H., Galarraga, E., DruckerColin, R., \& Bargas, J. (2009). Dopamine D2-class receptor supersensitivity as reflected in $\mathrm{Ca} 2+$ current modulation in neostriatal neurons. Neuroscience, 164(2), 345-350. https://doi.org/10.1016/j.neuroscience.2009.08.030

Rebec, G. V. (1998). Real-Time Assessments of Dopamine Function during Behavior: Single-Unit Recording, Iontophoresis, and Fast-Scan Cyclic Voltammetry in Awake, Unrestrained Rats. Alcoholism: Clinical and Experimental Research, 22(1), 32-40.

Richter, A., Xie, Y., Schumacher, A., Loeffler, S., Kirch, R., Al-Hasani, J., ... Hofmann, U. G. (2013). A simple implantation method for flexible, multisite microelectrodes into rat brains. Frontiers in Neuroengineering, 6. https://doi.org/10.3389/fneng.2013.00006

Rommelfanger, K. S. \& Wichmann, T. (2010). Extrastriatal dopaminergic circuits of the basal ganglia. Frontiers in Neuroanatomy, 4. https://doi.org/10.3389/fnana.2010.00139

Rubin, J. E., McIntyre, C. C., Turner, R. S., \& Wichmann, T. (2012). Basal ganglia activity patterns in parkinsonism and computational modeling of their downstream effects. European Journal of Neuroscience, 36(2), 2213-2228. https://doi.org/10.1111/j.1460-9568.2012.08108.x

Ruskin, D. N., Bergstrom, D. A., Mastropietro, C. W., Twery, M. J., \& Walters, J. R. (1999). Dopamine agonist-mediated rotation in rats with unilateral nigrostriatal lesions is not dependent on net inhibitions of rate in basal ganglia output nuclei. Neuroscience, 91(3), 935-946. 
Ryan, L. J., S. J. Young, and P. M. Groves. 1986. "Substantia Nigra Stimulation Evoked Antidromic Responses in Rat Neostriatum." Experimental Brain Research 63 (3): 44960 .

Ryan, L J, S J Young, D S Segal, and P M Groves. 1989. “Antidromically Identified Striatonigral Projection Neurons in the Chronically Implanted Behaving Rat: Relations of Cell Firing to Amphetamine-Induced Behaviors." Behavioral Neuroscience 103 (1): 314.

Rymar, Vladimir V., Rachel Sasseville, Kelvin C. Luk, and Abbas F. Sadikot. 2004. "Neurogenesis and Stereological Morphometry of Calretinin-Immunoreactive GABAergic Interneurons of the Neostriatum.” The Journal of Comparative Neurology 469 (3): 325-39. https://doi.org/10.1002/cne.11008.

Saka, E., M. Iadarola, D. J. Fitzgerald, and A. M. Graybiel. 2002. "Local Circuit Neurons in the Striatum Regulate Neural and Behavioral Responses to Dopaminergic Stimulation.” Proceedings of the National Academy of Sciences 99 (13): 9004-9009.

Sano, H., Chiken, S., Hikida, T., Kobayashi, K., \& Nambu, A. (2013). Signals through the Striatopallidal Indirect Pathway Stop Movements by Phasic Excitation in the Substantia Nigra. Journal of Neuroscience, 33(17), 7583-7594. https://doi.org/10.1523/JNEUROSCI.4932-12.2013

Schmitzer-Torbert, N.C., and A.D. Redish. 2008. "Task-Dependent Encoding of Space and Events by Striatal Neurons Is Dependent on Neural Subtype." Neuroscience 153 (2): 349-60. https://doi.org/10.1016/j.neuroscience.2008.01.081.

Schwarting, R., \& Huston, J. (1996). The unilateral 6-hydroxydopamine lesion model in behavioral brain research. Analysis of functional deficits, recovery and treatments.

Progress in Neurobiology, 50(2-3), 275-331. https://doi.org/10.1016/S0301$\underline{0082(96) 00040-8}$

Schwarz, Cornelius, and Shubhodeep Chakrabarti. 2015. "Whisking Control by Motor Cortex." Scholarpedia 10 (3): 7466. https://doi.org/10.4249/scholarpedia.7466.

Sharott, A., N. M. Doig, N. Mallet, and P. J. Magill. 2012. "Relationships between the Firing of Identified Striatal Interneurons and Spontaneous and Driven Cortical Activities In Vivo." Journal of Neuroscience 32 (38): 13221-36. https://doi.org/10.1523/JNEUROSCI.2440-12.2012.

Singh, B., Wilson, J. H., Vasavada, H. H., Guo, Z., Allore, H. G., \& Zeiss, C. J. (2007). Motor deficits and altered striatal gene expression in aphakia (ak) mice. Brain Research, 1185, 283-292. https://doi.org/10.1016/j.brainres.2007.09.006 
Singh, A., Liang, L., Kaneoke, Y., Cao, X., \& Papa, S. M. (2015). Dopamine regulates distinctively the activity patterns of striatal output neurons in advanced parkinsonian primates. Journal of Neurophysiology, 113(5), 1533-1544.

https://doi.org/10.1152/jn.00910.2014

Singh, A., Mewes, K., Gross, R. E., DeLong, M. R., Obeso, J. A., \& Papa, S. M. (2016). Human striatal recordings reveal abnormal discharge of projection neurons in Parkinson's disease. Proceedings of the National Academy of Sciences, 113(34), 9629-9634. https://doi.org/10.1073/pnas.1606792113

Sippy, T., Lapray, D., Crochet, S., \& Petersen, C. C. H. (2015). Cell-Type-Specific Sensorimotor Processing in Striatal Projection Neurons during Goal-Directed Behavior. Neuron, 88(2), 298-305. https://doi.org/10.1016/j.neuron.2015.08.039

Smith, K. S., \& Graybiel, A. M. (2013). A Dual Operator View of Habitual Behavior Reflecting Cortical and Striatal Dynamics. Neuron, 79(2), 361-374. https://doi.org/10.1016/j.neuron.2013.05.038

Smith, Y., \& Villalba, R. (2008). Striatal and extrastriatal dopamine in the basal ganglia: An overview of its anatomical organization in normal and Parkinsonian brains. Movement Disorders, 23(S3), S534-S547. https://doi.org/10.1002/mds.22027

Smits, S. M., Mathon, D. S., Burbach, J. P. H., Ramakers, G. M. J., \& Smidt, M. P. (2005). Molecular and cellular alterations in the Pitx3-deficient midbrain dopaminergic system. Molecular and Cellular Neuroscience, 30(3), 352-363. https://doi.org/10.1016/i.mcn.2005.07.018

Surmeier, D. James, Jun Ding, Michelle Day, Zhonfeng Wang, Weixing Shen, 2007. "D1 and D2 Dopamine-Receptor Modulation of Striatal Glutamatergic Signaling in Striatal Medium Spiny Neurons." Trends in Neurosciences 30 (5): 228-35.

https://doi.org/10.1016/j.tins.2007.03.008

Swadlow, H A, S G Waxman, and D L Rosene. 1978. "Latency Variability and the Identification of Antidromically Activated Neurons in Mammalian Brain." Experimental Brain Research 32 (3): 439-43.

Tepper, J. M. 2010. "GABAergic Interneurons of the Striatum." Handbook of Basal Ganglia Structure and Function, 151-166.

Tepper, J.M., and T. Koós. 2017. "GABAergic Interneurons of the Striatum.” In Handbook of Behavioral Neuroscience, 24:157-78. Elsevier. https://doi.org/10.1016/B978-0-12-802206-1.00008-8.

Tong, J., Fitzmaurice, P. S., Ang, L. C., Furukawa, Y., Guttman, M., \& Kish, S. J. (2004). Brain dopamine-stimulated adenylyl cyclase activity in Parkinson's disease, 
multiple system atrophy, and progressive supranuclear palsy. Annals of Neurology, 55(1), $125-129$.

Tseng, K. Y., Kasanetz, F., Kargieman, L., Riquelme, L. A., \& Murer, M. G. (2001). Cortical slow oscillatory activity is reflected in the membrane potential and spike trains of striatal neurons in rats with chronic nigrostriatal lesions. The Journal of Neuroscience: The Official Journal of the Society for Neuroscience, 21(16), 6430-6439.

Tseng, K. Y., Riquelme, L. A., \& Murer, M. G. (2004). Impact of D1-class dopamine receptor on striatal processing of cortical input in experimental parkinsonism in vivo. Neuroscience, 123(2), 293-298.

Turner, Robert S., and Mahlon R. DeLong. 2000. "Corticostriatal Activity in Primary Motor Cortex of the Macaque.” The Journal of Neuroscience 20 (18): 7096-7108.

Usiello, A., Baik, J.-H., Rougé-Pont, F., Picetti, R., Dierich, A., LeMeur, M., ... Borrelli, E. (2000). Distinct functions of the two isoforms of dopamine D2 receptors. Nature, 408(6809), 199-203. https://doi.org/10.1038/35041572

Valjent, E., Bertran-Gonzalez, J., Hervé, D., Fisone, G., \& Girault, J.-A. (2009). Looking BAC at striatal signaling: cell-specific analysis in new transgenic mice. Trends in Neurosciences, 32(10), 538-547. https://doi.org/10.1016/j.tins.2009.06.005

van den Munckhof, P., Gilbert, F., Chamberland, M., Levesque, D., \& Drouin, J. (2006). Striatal neuroadaptation and rescue of locomotor deficit by l-dopa in aphakia mice, a model of Parkinson's disease. Journal of Neurochemistry, 96(1), 160-170. https://doi.org/10.1111/j.1471-4159.2005.03522.x

Waszczak, B. L., Lee, E. K., Tamminga, C. A., \& Walters, J. R. (1984). Effect of dopamine system activation on substantia nigra pars reticulata output neurons: variable single-unit responses in normal rats and inhibition in 6-hydroxydopamine-lesioned rats. The Journal of Neuroscience, 4(9), 2369-2375.

Wei, W., Li, L., Yu, G., Ding, S., Li, C., \& Zhou, F.-M. (2013). Supersensitive presynaptic dopamine D2 receptor inhibition of the striatopallidal projection in nigrostriatal dopamine-deficient mice. Journal of Neurophysiology, 110(9), 2203-2216. https://doi.org/10.1152/jn.00161.2013

West, M. O., Carelli, R. M., Pomerantz, M., Cohen, S. M., Gardner, J. P., Chapin, J. K., \& Woodward, D. J. (1990). A region in the dorsolateral striatum of the rat exhibiting single-unit correlations with specific locomotor limb movements. Journal of Neurophysiology, 64(4), 1233-1246.

West, M. O. (1998). Anesthetics eliminate somatosensory-evoked discharges of neurons in the somatotopically organized sensorimotor striatum of the rat. The Journal of Neuroscience, 18(21), 9055-9068. 
Wichmann, T., \& DeLong, M. R. (1996). Functional and pathophysiological models of the basal ganglia. Current Opinion in Neurobiology, 6(6), 751-758.

Wilson, Charles J. 1987. "Morphology and Synaptic Connections of Crossed Corticostriatal Neurons in the Rat." The Journal of Comparative Neurology 263 (4): 56780. https://doi.org/10.1002/cne.902630408.

Xu, M., Moratalla, R., Gold, L. H., Hiroi, N., Koob, G. F., Graybiel, A. M., \& Tonegawa, S. (1994). Dopamine D1 receptor mutant mice are deficient in striatal expression of dynorphin and in dopamine-mediated behavioral responses. Cell, 79(4), 729-742.

https://doi.org/10.1016/0092-8674(94)90557-6

Xu, M., Koeltzow, T. E., Santiago, G. T., Moratalla, R., Cooper, D. C., Hu, X.-T., ... Tonegawa, S. (1997). Dopamine D3 Receptor Mutant Mice Exhibit Increased Behavioral Sensitivity to Concurrent Stimulation of D1 and D2 Receptors. Neuron, 19(4), 837-848. https://doi.org/10.1016/S0896-6273(00)80965-4

Xu, H., Chen, R., Cai, X., \& He, D. (2011). Differential effects of activating D1 and D2 receptors on electrophysiology of neostriatal neurons in a rat model of Parkinson's disease induced by paraquat and maneb. Neuroscience Research. Retrieved from http://www.sciencedirect.com/science/article/pii/S0168010211020645

Yung, K. K., Bolam, J. P., Smith, A. D., Hersch, S. M., Ciliax, B. J., \& Levey, A. I. (1995). Immunocytochemical localization of D1 and D2 dopamine receptors in the basal ganglia of the rat: light and electron microscopy. Neuroscience, 65(3), 709-730. 


\section{VITA}

I was born in 1984. After finally deciding on a science degree out of appreciation for the attention to detail and empiricism (the power that is knowledge), I took my time at Cal State Northridge; from 2004-2008 and beyond in the Minehan organic chemistry lab. I developed my fine motor skills, long-lasting and valuable friendships, and very few functional organic compounds. Nevertheless, my heart was set on pharmacology and the understanding of voluntary perception, the responsibility that adults hold for their perspective. Off to UTHSC for a whirlwind of electrophysiology, mouse work, and a hard knock lesson in political mechanics of academic misconduct. It's been quite a ride so far... Here's to another 130 years! (hopefully that's enough time to reclaim my rightful spot as father of gangsta rap guitar covers on video sharing sites.) 